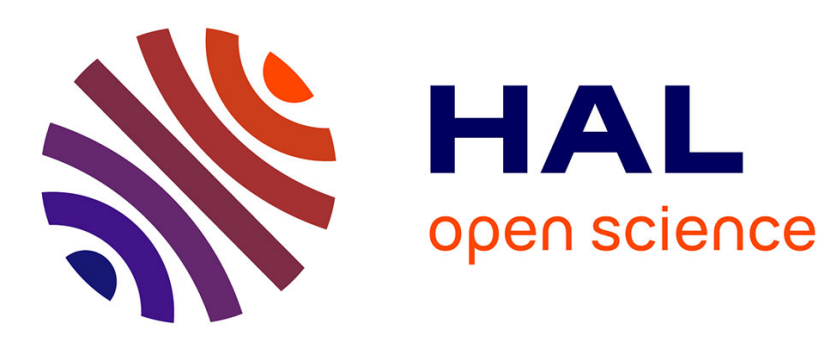

\title{
A mathematical model to investigate the key drivers of the biogeography of the colon microbiota.
}

Simon Labarthe, Bastien Polizzi, Thuy Phan, Thierry Goudon, Magali Ribot, Béatrice Laroche

\section{> To cite this version:}

Simon Labarthe, Bastien Polizzi, Thuy Phan, Thierry Goudon, Magali Ribot, et al.. A mathematical model to investigate the key drivers of the biogeography of the colon microbiota.. Journal of Theoretical Biology, 2019, 462 (7), pp.552-581. 10.1016/j.jtbi.2018.12.009 . hal-01761191v3

\section{HAL Id: hal-01761191 \\ https://hal.science/hal-01761191v3}

Submitted on 6 Dec 2018

HAL is a multi-disciplinary open access archive for the deposit and dissemination of scientific research documents, whether they are published or not. The documents may come from teaching and research institutions in France or abroad, or from public or private research centers.
L'archive ouverte pluridisciplinaire HAL, est destinée au dépôt et à la diffusion de documents scientifiques de niveau recherche, publiés ou non, émanant des établissements d'enseignement et de recherche français ou étrangers, des laboratoires publics ou privés. 


\title{
A mathematical model to investigate the key drivers of the biogeography of the colon microbiota.
}

\author{
Simon Labarthe ${ }^{\mathrm{a}, *}$, Bastien Polizzi ${ }^{\mathrm{b}}$, Thuy Phan ${ }^{\mathrm{c}}$, Thierry Goudon ${ }^{\mathrm{d}}$, Magali \\ Ribot $^{\mathrm{c}}$, Beatrice Laroche ${ }^{\mathrm{a}}$ \\ ${ }^{a}$ MaIAGE, INRA, Paris-Saclay University, Jouy-en-Josas, France \\ ${ }^{b}$ Univ Lyon, CNRS, Université Claude Bernard Lyon 1, UMR5208, Institut Camille Jordan, \\ F-69622 Villeurbanne, France \\ ${ }^{c} I D P$, Université d'Orléans-CNRS, Orleans, France \\ ${ }^{d}$ Université Côte d'Azur, Inria, CNRS, LJAD, Nice, France
}

\begin{abstract}
The gut microbiota, mainly located in the colon, is engaged in a complex dialogue with the large intestinal epithelium through which important regulatory processes for the health and well-being of the host take place. Imbalances of the microbial populations, called dysbiosis, are related to several pathological status, emphasizing the importance of understanding the gut bacterial ecology. Among the ecological drivers of the microbiota, the spatial structure of the colon is of special interest: spatio-temporal mechanisms can lead to the constitution of spatial interactions among the bacterial populations and of environmental niches that impact the overall colonization of the colon. In the present study, we introduce a mathematical model of the colon microbiota in its fluid environment, based on the explicit coupling of a population dynamics model of microbial populations involved in fibre degradation with a fluid dynamics model of the luminal content. This modeling framework is used to study the main drivers of the spatial structure of the microbiota, specially focusing on the dietary fibre inflow, the epithelial motility, the microbial active swimming and viscosity gradients in the digestive track. We found 1) that the viscosity gradients allow the creation of favorable niches in the vicinity of the mucus layer; 2 ) that very low microbial active swimming in the radial direction is enough to promote bacterial growth, which sheds a new light on microbial motility in the colon and 3) that dietary fibres are the main driver of the spatial structure of the microbiota in the distal bowel whereas epithelial motility is preponderant for the colonization of the proximal colon; in the transverse colon, fibre levels and chemotaxis have the strongest impact on the distribution of the microbial communities.
\end{abstract}

Keywords: mathematical model, PDE, gut microbiota, microbial ecology, population dynamics, fluid mechanics

\footnotetext{
* Corresponding author

Email address: simon.labarthe@inra.fr (Simon Labarthe)
} 


\section{Introduction}

Humans host in their colon a large community of symbiotic microorganisms, the gut microbiota. Complex ecological cross-talks between the microbial populations and the gut epithelium are involved in the regulation of this community, but also in the host homeostasis [37. Microbial population imbalances, called dysbiosis, are now associated with number of physiopathological status, such as metabolic, auto-immune, inflammatory or even mental diseases 34, 35. The microbial ecology of the gut is thus intensively studied in order to better understand the link between the gut microbiota and the host health and wellness by deciphering the mechanisms that shape the microbiota community structure.

Among them, the spatial organization of the microbiota plays an important role, both in the installation and maintenance of the microbiota, and impacts the host health, as recently outlined in 13 . The identification of the parameters that influence this spatial structure is of particular interest.

First, the colon is the place of complex fluid mechanics: the luminal flow of digestive residuals carries along the colonic content towards its distal part, while the colon epithelium pumps water $[3$, twisting the stream lines and reducing the transport speed. In the same time, the mucus layer that wraps the epithelium, together with the inhomogeneous luminal content, creates viscosity gradients that further deform the flow [19 while the active contractions of the intestine wall during its motile activity 24 induce additional perturbations. These interacting hydrodynamic and mechanic forces spatio-temporally structure the colon microbiota.

The second parameter impacting the spatial distribution of the microbiota is the nutrient availability. The colon is an anaerobic medium, where the main nutrient sources for microorganisms are undigested dietary fibers or host-derived polysaccharides and their by-products: this constitutes a selection pressure that favors fermentative microorganisms. The polysaccharides degradation is therefore central in the ecological interactions within the microbiota and structures the whole community through trophic exchanges of electron acceptors 13. The interplay between the microbial populations and their nutritional landscape can be further intricate due to their ability to forage for nutritional sources through active motion: whereas bacterial flagella expression is repressed by the host immune system near the epithelium [10], active swimming is needed for the colonization of several pathogens 13 and low motile activity is observed for commensal bacteria 42 .

Finally, the epithelial mucus plays a particular role in the gut microbiota homeostasis and its spatial shape. This viscous fluid insulates the epithelium wall and forms a passive protection against a microbial invasion. But it also provides an additional way for the bacteria to escape the flow of the intestinal content by binding to the mucus layer to prevent their wash out. Furthermore, the mucus represents a source of polysaccharides directly provided by the host: the mucins and their glycans that compose the epithelial mucus can be degraded with the same enzymatic mechanisms as for fibers 20.

Experimental devices mimicking the colonic environment provide highly valuable information [13, 26, 27, 41, 2, on the gut microbiota functioning and its spatial structure. Reductionist approaches on gnotobiotic rodents 43 are also highly valuable tools. However, they only partially mimic the host response or the ecosystem functions, making difficult to evaluate the relative importance 
of the factors that shape the spatial distribution of the microbiota. This is why mathematical modeling approaches provide a helpful complement to experiments to gain insight on the main parameters influencing the spatial structure of the bacteria in the colon.

Several models of the gut microbiota were proposed in the literature to study the spatial structure of the microbial communities. The first model that was introduced 29] emphasized the modeling of the fiber degradation activity, by adapting a model of anaerobic digestion from bioprocess engineering [4. The space was handled through a rough discretization of the colon into physiological compartments. An improved version of this model, based on an infinite sequence of longitudinal compartments represented by a one-dimensional partial differential equation was developed in 28. It assumed a constant flow speed along the colon, reducing the fluid mechanic effects to an averaged retention time. A more sophisticated description of the hydrodynamic transport speed was proposed in [8], together with a diffusive term describing the peristaltic activity of the large intestine assessed by comparison with biophysical experiments [9], and a $\mathrm{pH}$-dependant bacterial activity. The resulting model was unidimensional in space, and the hydrodynamics was reduced to the volume conservation during water absorption, while the gut microbial community was simplified up to a pair of bacterial strains. In [14], an accurate description of the fluid dynamics of the multiphasic luminal content was proposed to study the constitution and the turnover of the mucus layer, but the interactions with the microbiota were not studied.

In this paper, we present a new model coupling the fluid mechanics model of the colonic content and the mucus layer introduced in [14] with the metabolic model of bacterial populations presented in [29. To our knowledge, this model allows for the first time a full study of the spatial distribution of the microbiota including the interactions with its fluid environment. The complexification of the fluid mechanics description allows to investigate specific features such as epithelial motility, active swimming or the dependency of the local viscosity to the luminal content composition, together with their impact on the fluid streams and the microbiota growth.

The model we propose relies on reasoning and methods from mixture theory, which are described in Section 2. The impact of the leading biological mechanisms that govern the equations is investigated on numerical grounds in Section 3 We show numerically that the ecosystem reaches an equilibrium (Section 3.1), in coherence with behavior and orders of magnitude reported in the literature, that will be considered as a reference state for further comparison. Then, we discuss the influence of several parameters on the stability and the profile of this reference state (Section 3.2). We pay a specific attention to 1) diet variations which are taken into account by tuning the dietary fiber levels (Section 3.2.1), 2) heterogeneities in the fluid viscosity and bacterial mucus metabolism (Section 3.2.2), 3) the bacterial active motion and the epithelial motility (Section 3.2.3. A sensitivity analysis is conducted to decipher the impact of the different mechanisms (Section 3.2.4). Section 4 is devoted to a thorough discussion about the modeling assumptions, and issues raised by the validation of the model from experimental data. 


\section{Material and methods}

\subsection{Mathematical model of the gut microbiota and its metabolic substrate}

We present the mathematical framework, emphasizing the underlying biological assumptions of the model and the modeling of the different mechanisms able to impact the spatial structure of the microbiota.

\subsubsection{Geometrical assumptions}

The dynamic of the populations and their interaction with the fluid is described by a set of PDEs structured in space. The equations are set on a cylindrical domain $\Omega=\omega \times[0, L]$ of $\mathbb{R}^{3}$ that represents the geometry of the colon, with $\omega \subset \mathbb{R}^{2}$ its transverse section, which is a disc of radius $R>0$. We denote by

$$
\Gamma_{\text {in }}=\omega \times\{0\}, \quad \Gamma_{\text {out }}=\omega \times\{L\}, \quad \Gamma_{m}=\partial \Omega \backslash\left(\Gamma_{\text {in }} \cup \Gamma_{\text {out }}\right)
$$

the proximal extremity (just after the ileocaecal valve), the distal boundary (which is set in the middle of the sigmoid colon, before the rectum) and the mucosal wall of the colon, respectively. Physiological compartments can be identified as portions of the total cylinder: the proximal, transverse and distal colons have respective length of $L_{\text {prox }}, L_{\text {trans }}, L_{\text {dist }}$, which satisfy $L_{\text {prox }}+L_{\text {trans }}+$ $L_{d i s t}=L$. In our approach, the end of the sigmoid and the rectal parts are not modeled. More precisely we take $L_{\text {prox }}=21 \mathrm{~cm}, L_{\text {dist }}=63 \mathrm{~cm}, L_{\text {trans }}=70 \mathrm{~cm}$ such that the total length is shorter than the averaged physiological colorectal length, which is about $190 \mathrm{~cm} 22,8$.

We refer the reader to Table Appendix A.4 for the physical values attributed to the geometrical data for the simulations.

\subsubsection{Global structure of the model}

The model we propose is based on reasonings from mixture theory [32, 7], adapted to the multiphasic intestinal content. We refer the reader to [14] for a first attempt in that direction. Among the different entities considered in this model, we distinguish the mixture components that are large enough to produce mechanical forces, hence impacting the fluid mechanics, and the diffusing compounds dissolved in the intestinal mixture, without any impact on the mixture flow. To determine the different metabolic elements to be included in the model, we follow the strategy introduced in 29: we assume that the fibers, which are the predominant source of raw material for metabolic activity, are determinant for the spatial organization of the microbiota and we consider the different metabolites involved in the fiber degradation in the colon as discussed in 29 .

The model involves four functional microbial metapopulations, each acting at different stages of polysaccharides metabolic pathways. The first population, called $\mathrm{B}_{m o n}$, hydrolyses the fibers and mucus polysaccharides to produce monosaccharides, that, in turn, are metabolized to support their growth, producing lactate, SCFA (acetate, propionate and butyrate) and dissolved gas $\left(\mathrm{H}_{2}\right.$ and $\left.\mathrm{CO}_{2}\right)$. The population $\mathrm{B}_{l a}$ then grows on lactate and produces shortchain fatty acids - SCFA - and gas, while the populations $\mathrm{B}_{H_{2} a}$ and $\mathrm{B}_{H_{2} m}$ are fueled by the di-hydrogen, through respectively the homeoacetogenesis and methanogenesis pathways. In order to maintain physiological gas concentration in the liquid, we model vaporisation to gaseous phase. We finally get 
13 processes (see Fig. Appendix A.1 for a synoptic view of the reactions involved in the model). The different bacterial populations are gathered in the set $I_{\mathrm{B}}=\left\{\mathrm{B}_{m o n}, \mathrm{~B}_{l a}, \mathrm{~B}_{H_{2} a}, \mathrm{~B}_{H_{2} m}\right\}$. Next, we assume that the larger elements capable to influence the fluid mechanics are the mucus $(m)$, the polysaccharides $(p o l)$, the 4 bacteria (described by the set $I_{\mathrm{B}}$ ), the liquid chyme $(l)$ and indigestible residuals $(r)$ that are not metabolized by bacteria but do impact the local rheology. We thus collect these mixture components in the 8 element set $I_{\mathrm{C}}=\left\{m, p o l, \mathrm{~B}_{m o n}, \mathrm{~B}_{l a}, \mathrm{~B}_{H_{2} a}, \mathrm{~B}_{H_{2} m}, l, r\right\}$, which thus contains $I_{\mathrm{B}}$. The dissolved compounds (which include, among other, the gas and SCFA involved in the model) are collected in the set $I_{\mathrm{S}}=\left\{\right.$ mon, la, $\mathrm{H}_{2}$, ac, pro, bu, $\left.\mathrm{CH}_{4}, \mathrm{CO}_{2}\right\}$ for respectively the monosaccharides, the lactate, the hydrogen, the acetate, the propionate, the butyrate, the methane and the carbone dioxyde. We remark that, unlike 29], we do not explicitly introduce a gaseous phase in the model.

In what follows, we describe in details the mathematical model:

- In Section 2.1.3, we introduce the convection-diffusion-reaction equations that govern the volume fraction of the intestinal mixture components and the concentration of dissolved components (see §Mass conservation). The velocity field for the mixture components is the sum of the velocity of the carrying fluid and a chemotactic field for the bacteria. The dissolved components are convected with the mean volume velocity of the mixture, which satisfies an incompressibility condition (see $§$ Velocity fields). This condition appears as a constraint, that completes the fluid mechanical equation for the velocity/pressure fields describing the carrying fluid (see $\S$ Stokes model for the average fluid motion).

- In Section 2.1.4 we detail the bacterial activity. We first detail the microbial active motion, which depends on the considered species (see $\S$ Microbial active motion). We next focus on the bacterial metabolism, which is addressed through stoichiometrically-balanced reaction terms(see $\S$ Microbial metabolic activity).

- In Section 2.1.5, we describe the interaction between the fluid dynamics and the environment imposed by the colon physiology. We begin by introducing boundary conditions that describe mass transfer mechanisms (absorption and release) by the gut epithelium, driving the overall transit (see §Mass transfers through the boundaries.). Next, we introduce a viscosity profile depending on the local composition of the mixture including the mucus excreted by the epithelial cells. This is a crucial feature of the proposed model, intended to take into account slow down mechanisms near the mucus layer (see $\S$ Definition of the viscosity). Finally, we detail the peristalsis and the gut wall motility, introduced in the model through boundary condition of the Stokes model (see §Epithelial motility and peristalsis).

- It turns out that the model can be simplified by means of asymptotic arguments, using the fact that the aspect ratio of the gut is a small parameter. The reduced model proposed in Section 2.2 is particularly relevant for numerical purposes, since it greatly decreases the computational cost.

The sketch of the model presentation is summarized in Figure 1. 


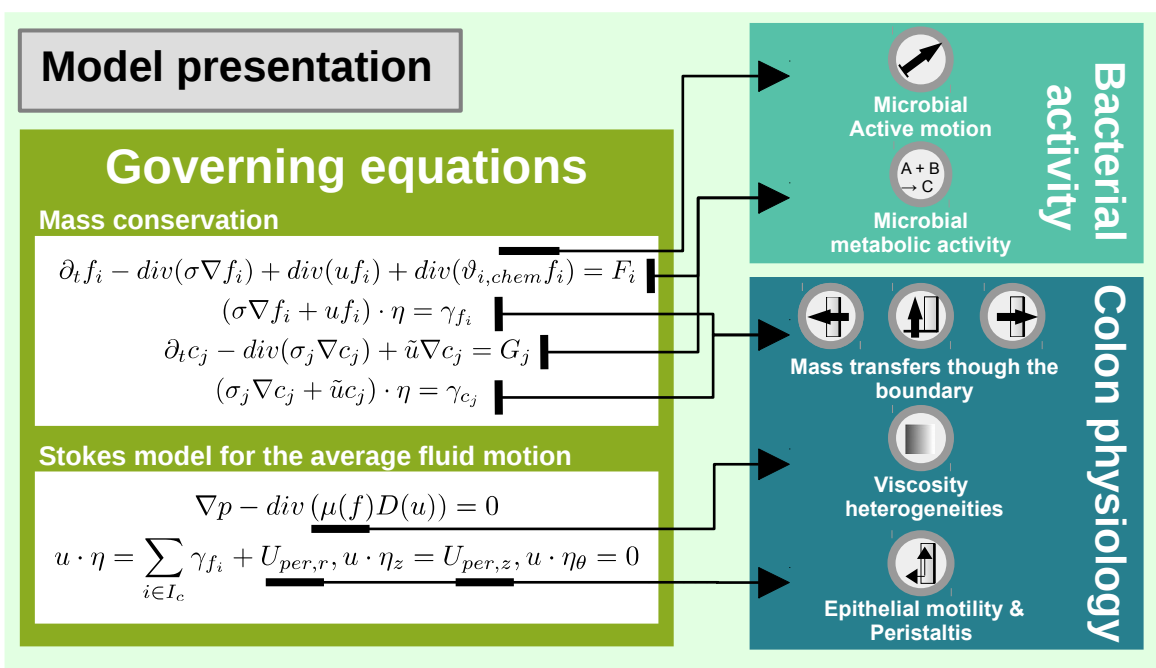

Figure 1: Presentation of the mathematical model. First, the governing equations are introduced in 2.1.3 Then, the terms modeling the bacterial functions are detailed in 2.1.4 Finally, the interactions between colon physiology and fluid dynamics are described in 2.1 .5

\subsubsection{Governing equations}

Mass conservation.. As all the phases of the multiphasic colonic content are mainly composed of water, we assume that they all have the same constant mass density $\rho$ (see Table Appendix A.4). The mixture state is then totally described by the volume fractions $f_{i}$ of its phases. We model the time evolution of the volume fraction of the component $i \in I_{\mathrm{C}}$ by the following reaction-diffusionconvection equation

$$
\partial_{t} f_{i}-\operatorname{div}\left(\sigma \nabla f_{i}\right)+\operatorname{div}\left(f_{i} u_{i}\right)=F_{i}
$$

For simplification purposes, we assume the same diffusion coefficient $\sigma>0$ for all phases, but each phase has its own velocity field $u_{i}$. When the mixture is at rest, which means when the different transport terms vanish, the phases are supposed to inter-penetrate each other by diffusion: we assume that the interface forces are not sufficient to maintain a sharp separation of the different phases. As this diffusive process is supposed to be small comparatively to the transport process, we model that feature with the diffusive term $\operatorname{div}\left(\sigma \nabla f_{i}\right)$, a simple Fick's law, where $\sigma$ is a uniform diffusion coefficient that does not depend on the mixture phase. The definition of the source term $F_{i}$, the metabolic transformation rate of the mixture component $i$, will be detailed later on. Nevertheless, for the modeling issues, it is important to bear in mind that the phase-to-phase transfers embodied into the $F_{i}$ 's are volume invariant which amounts to assume that

$$
\sum_{i \in I_{\mathrm{C}}} F_{i}=0
$$

For the derivation of the equations, we are also going to use the fact that the mixture fills up the whole intestinal volume resulting in the following saturation 
constraint

$$
\sum_{i \in I_{\mathrm{C}}} f_{i}(t, x)=1, \quad \text { for any } t>0, x \in \Omega
$$

We equally use reaction-diffusion-convection equations for the dissolved compounds concentrations. However, we assume different diffusion coefficients while the velocity field for the dissolved constituents is defined by the local weighted average of the mixture phase velocities

$$
\tilde{u}=\sum_{i \in I_{\mathrm{C}}} f_{i} u_{i}
$$

(which can be interpreted as the mean volume velocity of the flow). We finally get the following equation for the concentration of the chemical $j$

$$
\partial_{t} c_{j}-\operatorname{div}\left(\sigma_{j} \nabla c_{j}\right)+\operatorname{div}\left(c_{j} \tilde{u}\right)=G_{j}
$$

where $\sigma_{j}>0$ and $G_{j}$ are the diffusion coefficient of the diffusing compound $j$ and its reaction rate, respectively. We emphasize that $G_{j}$ can gather several reaction rates if the compound $j$ is involved in several reactions (see Table Appendix A.1 and section Appendix A.1 in the Annexes for further details). The definition of the source term $G_{j}$ will also be precisely defined later on.

Velocity fields.. The convection of each fluid component $f_{i}$ results from two different phenomena:

- the transport by the carrying fluid, described by the velocity field $(t, x) \mapsto$ $u(t, x)$, the evolution of which is driven by fluid mechanics principles,

- for the bacteria, a correction which is intended to describe an active motion towards metabolite sources. This correction is modeled by a chemotactic velocity $(t, x) \mapsto \vartheta_{i, c h e m}(t, x)$, see [21, 16].

Therefore, we are led to define the apparent velocity field

$$
u_{i}=u+\vartheta_{i, \text { chem }}
$$

Using (3) and (4), this gives the following velocity for the dissolved compounds mass equation :

$$
\tilde{u}=u+\sum_{i \in I_{\mathrm{B}}} f_{i} \vartheta_{i, c h e m}
$$

It remains to detail the equations that govern the evolution of $u$ and $\vartheta_{i, c h e m}$.

Stokes model for the average fluid motion.. Summing Eq. (1) over $i \in I_{\mathrm{C}}$, together with Eq. (2) and Eq. (4) (or Eq. (6)), we get a constraint on the mean volume velocity

$\operatorname{div}\left(\sum_{i \in I_{\mathrm{C}}} f_{i} u_{i}\right)=\operatorname{div}(\tilde{u})=0 \quad$ or, equivalently, $\operatorname{div}(u)=-\operatorname{div}\left(\sum_{i \in I_{\mathrm{B}}} f_{i} \vartheta_{i, \text { chem }}\right)$.

This contraint enables us to write Eq.(5) as follows :

$$
\partial_{t} c_{j}-\operatorname{div}\left(\sigma_{j} \nabla c_{j}\right)+\tilde{u} \cdot \nabla c_{j}=G_{j}
$$


We end up with a momentum conservation equation: we model the mixture velocity with the following Stokes equation on $u$

$$
\nabla p-\operatorname{div}(\mu D(u))=0
$$

where $D(u)=\frac{1}{2}\left(\nabla u+\nabla u^{\top}\right)$ and $p$ is the pressure, that is to say the Lagrange multiplier which ensures the effectivity of the constraint (7). In this expression, $\mu$ is the apparent mixture viscosity, which depends on space and time through volume fractions. The definition of the viscosity leads to space inhomogeneities which are crucial for the modeling (see Sec. 2.1.5, §Viscosity heterogeneities).

It could be possible to incorporate in the right hand side or in the boundary condition a description of further mechanical effects. This raises interesting and delicate modeling issues, addressed for instance in the description of blood flows or respiration flows [30.

\subsubsection{Bacterial activity}

Microbial active motion.. The chemotactic potential describes the ability of the bacteria to move, independently of the flow of the carrying fluid.

Each bacteria is endowed by a chemotactic behavior directed towards their substrate: bacteria $\mathrm{B}_{m o n}$ is attracted by mucus, polysaccharides and monosaccharides, bacteria $\mathrm{B}_{l a}$ is attracted by lactate and $\mathrm{B}_{H_{2} a}$ and $\mathrm{B}_{H_{2} m}$ are attracted by dihydrogen.

For each bacteria $i \in I_{\mathrm{B}}$, the corresponding active motion is modeled by the Keller-Segel model: the gradient of a chemotactic potential influences the resulting velocity, which is therefore defined as

$$
\vartheta_{i, \text { chem }}=\sum_{j} \lambda_{i j} \nabla \Phi_{j}
$$

where $\Phi_{j}$ is the chemotactic potential created by the metabolite $j$ and $\lambda_{i, j}$ is the chemosensitivity coefficient for the bacteria $i$ and the metabolite $j$, see [21, 16]. In order to ease notations, we mention a chemotactic speed for all the mixture components in the model equations, bearing in mind that this speed is set to zero except for bacteria.

The chemotactic potential of the metabolite $j \in I_{\mathrm{S}} \cup\{m$, pol $\}$ is defined (up to an irrelevant constant) through the resolution of the Poisson equation with Neumann boundary conditions: with $\eta$ the unit outward normal vector on $\partial \Omega$, we have, when $j \in I_{\mathrm{S}}$,

$$
\begin{aligned}
-\Delta \Phi_{j}=c_{j}-\frac{1}{|\omega|} \int_{\omega} c_{j}(x, z) \mathrm{d} x & \text { in } \Omega \\
\nabla \Phi_{j} \cdot \eta=0 & \text { on } \partial \Omega .
\end{aligned}
$$

and, when $j \in\{m, p o l\}$,

$$
\begin{aligned}
-\Delta \Phi_{j}=f_{j}-\frac{1}{|\omega|} \int_{\omega} f_{j}(x, z) \mathrm{d} x & \text { in } \Omega \\
\nabla \Phi_{j} \cdot \eta=0 & \text { on } \partial \Omega .
\end{aligned}
$$

The term $\frac{1}{|\omega|} \int_{\omega} c_{j}(x, z) \mathrm{d} x\left(\operatorname{resp} \cdot \frac{1}{|\omega|} \int_{\omega} f_{j}(x, z) \mathrm{d} x\right)$, which averages over the transverse section $\omega$, relies on a compatibility condition for Eq. (11) (resp. (12) 
to be solvable. It differs from the usual average which involves the whole domain, that is to say $\frac{1}{|\Omega|} \int_{\Omega} c_{j}(x, z) \mathrm{d} x \mathrm{~d} z$ (resp. $\left.\frac{1}{|\Omega|} \int_{\Omega} f_{j}(x, z) \mathrm{d} x \mathrm{~d} z\right)$. This modification is motivated by the simplified model that we propose, based on asymptotic arguments, when the aspect ratio of the colon goes to 0, see Section 2.2. In the asymptotic limit, the longitudinal chemotactic forces vanish, and the operator $\Delta \Phi_{j}$ degenerates to $\frac{1}{r} \partial_{r}\left(r \partial_{r} \Phi_{j}\right)$ : the chemotactic active swimming occurs in the radial direction only. The term $\frac{1}{|\omega|} \int_{\omega} c_{j}(x, z) \mathrm{d} x\left(\operatorname{resp} \cdot \frac{1}{|\omega|} \int_{\omega} f_{j}(x, z) \mathrm{d} x\right)$ enables us to provide a compatibility condition which applies equally in the asymptotic regime of Eq. (11) (resp. (12)).

Microbial metabolic activity.. We note $P_{c}\left(\right.$ resp. $\left.P_{s}\right)$ the Petersen reaction $8 \times 13$ matrix for the 8 mixture components (resp. the 8 solutes) that defines the yield of each of the 13 processes on the corresponding compounds, based on stoichiometry [29]. We also introduce the kinetic rate vector $K=\left(K_{p}\right)_{p=P 1, \ldots, P 13}$, which components are defined by $k_{p} \phi_{p}$ for the different processes $p=P 1, \ldots, P 13$ under consideration, where $k_{p}$ represents a unitary maximal kinetic rate whereas $\phi_{p}$ models saturation effects. Finally, with $F=\left(F_{i}\right)_{i \in I_{\mathrm{C}}}$ and $G=\left(G_{j}\right)_{j \in I_{\mathrm{S}}}$, we set

$$
F=P_{c} K \text { and } G=P_{s} K .
$$

To ensure the volume-conservation condition (2), we consider that mucus or polysaccharides consumption, or bacterial death, releases an equivalent volume of liquid (see volume transfers in Fig. Appendix A.1. Conversely, we assume that bacterial growth is limited by the available free space in the liquid phase (see Table Appendix A.1 in the annexes for the kinetic rates of each process), and that an equivalent volume of liquid is removed during the growth, in order to satisfy (2). Following [29], an additional pH-dependent-repression is introduced for the methanogens $\mathrm{B}_{H_{2} m}$ through a space-dependent linear $\mathrm{pH}$ function $p H(z):=I_{p H, \text { min }}+\left(I_{p H, \text { max }}-I_{p H, \text { min }}\right) z / L$ and a multiplicative factor $I_{p H}(z)$ applied to $k_{P_{6}} \phi_{P_{6}}$, where

$$
I_{p H}(z):=\exp \left(-3\left(\frac{p H(z)-I_{p H, \text { high }}}{I_{p H, \text { high }}-I_{p H, \text { low }}}\right)^{2}\right) \mathbf{1}_{p H(z)<I_{p H, \text { high }}}+\mathbf{1}_{p H(z) \geq I_{p H, \text { high }}}
$$

In this equation, we take the expression of pH-related repression of methanogenesis introduced in 29]: the methanogenesis is not repressed for $\mathrm{pH}$ higher than a threshold $I_{p H, h i g h}$, but faces an exponential repression for more acidic media. In 29], which was a compartmental model of the colon, three different $\mathrm{pH}$ levels were defined for the proximal, transverse and distal colons. We model this variation through $p H(z)$, i.e. a linear evolution between the proximal and distal $\mathrm{pH}$ values introduced in 29 .

The bacteria are assigned a constant death rate. We finally recall that, unlike 29, the gaseous phase is not modeled, preventing the introduction of an equilibrium term between the dissolved gas and its corresponding gaseous phase through an Henry law. We then introduce a sink source term $G_{j}:=$ $k_{i}\left(c_{j}-K_{h, P_{i}} R T\left[c_{j_{g}}\right]_{\infty}\right)$, for $j \in\left\{\mathrm{CH}_{4}, \mathrm{CO}_{2}, \mathrm{H}_{2}\right\}$ (see also Table Appendix A.1b), where $\left[c_{j_{g}}\right]_{\infty}$ is the asymptotic value of the corresponding gas in the proximal luminal part of [29], and $R$ and $T$ are the perfect gas constant and the temperature. It is equivalent to define a Henry law with a stationary homogeneous gas phase. 
We gather in Table Appendix A.1 the precise definition of the reaction matrices and the growth rates. The values for all the parameters are given in Table Appendix A.5.

\subsubsection{Colon physiology and its impact on the fluid dynamics}

Mass transfers through the boundaries.. The modeling of the mass transfers through the boundaries is a key step since they account for the lumen-epithelium exchanges, which are central both in the fluid dynamics and in the metabolic activity through metabolite absorption and release. They influence the longitudinal speed, as it will be emphasized in the formula 25] describing the transit motion in Section 2.2. Furthermore, the mass transfers also include the dietary intake, and in particular the fiber intake which is the main source of nutrients for the microbiota.

In order to model these transfers, we introduce boundary fluxes $\gamma_{f_{i}}$ and $\gamma_{c_{j}}$ and supplement the mass conservation equations (1) and (8) by the natural Robin boundary conditions:

$$
\left(-\sigma \nabla f_{i}+f_{i} u\right) \cdot \eta=\gamma_{f_{i}} \quad \text { and } \quad\left(-\sigma_{j} \nabla c_{j}+c_{j} u\right) \cdot \eta=\gamma_{c_{j}} \quad \text { on } \partial \Omega,
$$

where $\eta$ is the unit outward normal vector to the boundary. Note that since the chemotactic field vanishes at the boundaries, we have that $u \cdot \eta=\tilde{u} \cdot \eta$ on $\partial \Omega$.

It remains to detail the boundary fluxes $\gamma_{f_{i}}$ and $\gamma_{c_{j}}$. We distinguish several cases where $\gamma_{f_{i}}$ and $\gamma_{c_{j}}$ are some constant values, or functions of the space variables, or functions of the phase volume fractions.

We recall here that $\Gamma_{i n}$ denotes the proximal extremity, $\Gamma_{\text {out }}$ the distal extremity of the colon and $\Gamma_{m}$ the mucosal wall of the large intestine, that is to say the lateral boundary.

- Dietary inflow on $\Gamma_{i n}$. We introduce a velocity profile $u_{i n}=u \cdot \eta$ on $\Gamma_{i n}$ such that its average is equal to $U_{i n}=V_{i n} /|\omega|$ where $V_{i n}$ is the daily volume of digestat that reaches the colon. The dietary inflow of fibers, bacteria and monosaccharides is then defined with the formula $\gamma_{f_{i}}=U_{i n} f_{i, \text { in }}$ on $\Gamma_{i n}$, where $f_{i, i n}$ is the component density in the inflow. Similarly, we will set $\gamma_{c_{j}}=U_{i n} c_{j, i n}$ on $\Gamma_{i n}$

Values $U_{i n}, f_{i, i n}$ and $c_{j, i n}$ are given in agreement with biological observations, see Table Appendix A.6. Note that the coefficients $c_{l a, i n}, c_{a c, i n}$, $c_{b u, i n}, c_{\text {pro,in }}, c_{H_{2}, i n}, c_{C H_{4}, i n}, c_{C O_{2}, i n}$ are all equal to $0 \mathrm{~mol}_{\mathrm{c}} \mathrm{cm}^{-3}$.

- Water pumping through the mucosa on $\Gamma_{m}$. According to [8, we define the strongest water pumping rate $g_{l, \max }$ in the proximal part of the colon mucosa, followed by a linear diminution of the water uptake, and finally a smaller basal activity $g_{l, \text { min }}$ on the distal part. We introduce $L_{\text {prox }}^{\text {water }}$ and $L_{\text {dist }}^{\text {water }}$ as the limit of application of $g_{l, \max }$ and $g_{l, \text { min }}$. We define on $\Gamma_{m}$

$$
\begin{aligned}
& \gamma_{f_{l}}=g_{l, \max } f_{l} \quad \text { for } z \in\left[0, L_{\text {prox }}^{\text {water }}\right], \\
& \gamma_{f_{l}}=\left(g_{l, \text { max }}-\frac{\left(z-L_{\text {prox }}^{\text {water }}\right)\left(g_{l, \text { max }}-g_{l, \text { min }}\right)}{L_{\text {dist }}^{\text {water }}-L_{\text {prox }}^{\text {water }}}\right) f_{l} \text { for } z \in\left[L_{\text {prox }}^{\text {water }}, L_{\text {dist }}^{\text {water }}\right], \\
& \gamma_{f_{l}}=g_{l, \text { min }} f_{l} \quad \text { for } z \in\left[L_{\text {dist }}^{\text {water }}, L\right] .
\end{aligned}
$$


- Mucus production on $\Gamma_{m}$. We consider that the mucosa insures the mucus layer homeostasis by a regulatory mechanism that produces mucus when the mucus level is below a threshold $f_{m}^{*}$ and reduces mucus level otherwise. We then set on $\Gamma_{m}$

$$
\gamma_{f_{m}}=g_{m}\left(f_{m}-f_{m}^{*}\right)
$$

where $g_{m}$ is the mucus production rate.

On $\Gamma_{m}$, we also set $\gamma_{f_{i}}=0$ when $i \in I_{\mathrm{C}} \backslash\{l, m\}=\left\{p o l, r, \mathrm{~B}_{m o n}, \mathrm{~B}_{l a}, \mathrm{~B}_{H_{2} a}, \mathrm{~B}_{H_{2} m}\right\}$.

- SCFA and other compounds absorption on $\Gamma_{m}$. On $\Gamma_{m}$ we impose a linear distribution of SCFA absorption along the mucosal wall between a maximal absorption rate $g_{j, \max }$ in the proximal part and a minimal rate $g_{j, \min }$ in the distal part, for the SCFA $j$. We then set

$$
\gamma_{c_{j}}=g_{j, \max }-\left(g_{j, \max }-g_{j, \min }\right) \frac{z}{L} \text { for } j \in\{l a, a c, \text { pro }, b u\}
$$

and

$$
\gamma_{c_{j}}=0 \text { otherwise, that is to say for } j \in\left\{\text { mon, } \mathrm{H}_{2}, \mathrm{CH}_{4}, \mathrm{CO}_{2}\right\} .
$$

- Outflow on $\Gamma_{\text {out }}$. Summing the mass conservation equations (1) over $i \in I_{\mathrm{C}}$, taking into account the saturation constraint (3), the volume conservation constraint (2) and the boundary conditions (14), and integrating over $\Omega$ shows that

$$
\int_{\Gamma_{\text {out }}} u \cdot \eta \mathrm{d} \sigma=\sum_{i \in I_{\mathrm{C}}} \int_{\Gamma_{i n} \cup \Gamma_{m}} \gamma_{f_{i}} \mathrm{~d} \sigma .
$$

In other words, the outflow balances the other mass transfers through the boundaries in order to conserve the overall volume. We then set on $\Gamma_{\text {out }}$, for all $i \in I_{\mathrm{C}}, \gamma_{f_{i}}=f_{i} u_{i} \cdot \eta$ and for all $j \in I_{\mathrm{S}}, \gamma_{c_{j}}=c_{j} \tilde{u} \cdot \eta=c_{j} u \cdot \eta$.

Viscosity heterogeneities.. We assume that the local viscosity is inhomogeneous and depends on the local composition of the mixture. This introduces a strong coupling between the fluid components and the velocity field. We consider that the main drivers of the local mixture viscosity are the mucus and the liquid chyme volume fractions. We set

$$
\mu(x, z, t)=\max \left(\mu_{m}\left(f_{m}(x, z, t)\right), \mu_{l}\left(f_{l}(x, z, t)\right)\right)
$$

where $\mu_{m}$ (resp. $\mu_{l}$ ) stands for a function describing the mucus rheology (resp. the luminal rheology) and depending on the mucus volume fraction (resp. the liquid volume fraction).

Following [14], we first sketch the highly viscous gel-like mucus layer by defining $\mu_{m}$ as a sigmoid function. A threshold level of mucine $f_{m, t h r}$ is defined as a marker of the mucus layer: above this threshold, we consider that the mixture is actually mucus and it is assigned a value close to the mucus viscosity $\mu_{m, \max }$. Under this level, the contribution of $\mu_{m}$ to the overall viscosity is close to a small value $\mu_{m, m i n}$. The transition between both values is tuned by a parameter $\alpha_{m}$. Namely, we set

$$
\mu_{m}\left(f_{m}\right)=\mu_{m, \min }+\left(\mu_{m, \max }-\mu_{m, \min }\right) \frac{f_{m}^{\alpha_{m}}}{f_{m, t h r}^{\alpha_{m}}+f_{m}^{\alpha_{m}}} .
$$


The luminal rheology is defined in the same way based on the liquid phase $l$ : the more liquid $l$, the less viscous is the mixture, which leads to

$$
\mu_{l}\left(f_{l}\right)=\mu_{l, \max }-\left(\mu_{l, \max }-\mu_{l, \min }\right) \frac{f_{l}^{\alpha_{l}}}{f_{l, t h r}^{\alpha_{l}}+f_{l}^{\alpha_{l}}} .
$$

We choose the parameters of the sigmoidal functions so that the sharp transition between the minimal and maximal values of the viscosity occurs in the interval $(0,1)$, where the volume densities $f_{m}$ and $f_{l}$ belong to.

Epithelial motility and peristalsis.. To close the overall system, it only remains to define boundary conditions for the velocity on $\Gamma_{m}$. We remind the reader $\eta$ as the local unitary outgoing normal vector, and we denote by $\eta_{z}$ the longitudinal tangential unitary vector (that is $\eta_{z}=(0,0,1)$ on $\Gamma_{m}$ and $\eta_{z}=(1,0,0)$ on $\Gamma_{\text {in }} \cup \Gamma_{\text {out }}$, in cylindrical coordinates), and $\eta_{\theta}=\eta_{z} \wedge \eta$ the radial tangential unitary vector. Then, we set

$$
\begin{gathered}
u \cdot \eta=\sum_{i \in I_{\mathrm{C}}} \gamma_{f_{i}}+U_{p e r, r}, \quad u \cdot \eta_{z}=U_{p e r, z}, \quad u \cdot \eta_{\theta}=0 \text { on } \Gamma_{m}, \\
u \cdot \eta=\sum_{i \in I_{\mathrm{C}}} \gamma_{f_{i}}, \quad u \cdot \eta_{z}=0, \quad u \cdot \eta_{\theta}=0 \text { on } \Gamma_{\text {in }} \cup \Gamma_{\text {out }},
\end{gathered}
$$

where $U_{\text {per }}=\left(U_{\text {per }, r}, 0, U_{\text {per }, z}\right)$ is a velocity profile describing the net motile activity of the mucosal wall, including peristalsis and segmentation contractions.

\subsubsection{Units of the model}

The time and space values are expressed in day and $\mathrm{cm}$. The mixture components are dimensionless, since they represent volume fractions and therefore $F_{i}$ should be in day $^{-1}$. Since we assume that all the phases have the same water density $\rho_{f_{i}}=1 \mathrm{~g} . \mathrm{cm}^{-1}$, the volume fractions can be easily converted to mass densities. To allow comparisons with the usual units of bacterial levels in microbiology literature, such as Colony Forming Units per grams (CFU.g ${ }^{-1}$ ) which is linked to the number of living microbes per mass unit, we assume that the average volume of a single bacteria is $1 \mu \mathrm{m}^{3}=10^{-12} \mathrm{~cm}^{3}$. Thus, a direct conversion between bacterial volume fractions and CFU.g ${ }^{-1}$ of colonic content can be obtained by applying a multiplicative factor of $10^{12}$ to the bacterial volume fraction. The densities of the dissolved compounds are expressed in mol.cm ${ }^{-3}$ The units of the different model parameters are detailed in Tables Appendix A.4 Appendix A.5 and Appendix A.6.

\subsection{Model simplification}

For computational purposes, it is interesting to work with a reduced model, which can be obtained owing to scaling reasoning. Using cylindrical coordinates $(r, \theta, z)$, we start by assuming that the state of the system does not depend on the angular coordinate $\theta$. Exploiting the aspect ratio of the colon $\varepsilon:=L / R \ll 1$, we formally expand the solutions of (1)-(18) as power series

$$
\begin{aligned}
& f_{i}=f_{i}^{(0)}+\varepsilon f_{i}^{(1)}+\varepsilon^{2} f_{i}^{(2)}+\ldots, \\
& c_{j}=c_{j}^{(0)}+\varepsilon c_{j}^{(1)}+\varepsilon^{2} c_{j}^{(2)}+\ldots, \\
& u=u^{(0)}+\varepsilon u^{(1)}+\varepsilon^{2} u^{(2)}+\ldots
\end{aligned}
$$


The asymptotics involves the differential operators

$$
u=\left(u_{r}, u_{z}\right) \mapsto \operatorname{div}_{r} u:=\frac{1}{r} \partial_{r}\left(r u_{r}\right)+\partial_{z}\left(u_{z}\right)
$$

and

$$
c \mapsto \nabla_{r} c=\left(\partial_{r} c, \partial_{z} c\right) .
$$

Identifying the leading terms in the expansion, we are led to

$$
\begin{gathered}
\sum_{i \in I_{\mathrm{C}}} f_{i}=1 \\
\partial_{t} f_{i}-\frac{1}{r} \partial_{r}\left(r \sigma \partial_{r} f_{i}\right)+\operatorname{div}_{r}\left(u_{s} f_{i}\right)+\frac{1}{r} \partial_{r}\left(r \vartheta_{i, r} f_{i}\right)=F_{i} \\
\partial_{t} c_{j}-\frac{1}{r} \partial_{r}\left(r \sigma_{j} \partial_{r} c_{j}\right)+\tilde{u}_{s} \cdot \nabla_{r} c_{j}=G_{j},
\end{gathered}
$$

where the velocities $u_{s}, \tilde{u}_{s}$ and the active motion field $\vartheta_{i, r}$ will be detailed below. We note that longitudinal diffusion and chemotactic velocity vanish, because the dimensional analysis reveals that these two contributions are negligible in comparison with the longitudinal transport. From a biological point of view, this is reminiscent to the assumption that the bacteria are not able to swim against the longitudinal flow, but that their active motion capabilities allow them to move along the radial direction.

The active transport velocity $\vartheta_{i, r}$ and the average speed for the diffuse compounds $\tilde{u}_{s}$ are given by

$$
\vartheta_{i, r}=\left(\sum_{j} \lambda_{i, j} \Upsilon_{j}, 0\right), \quad \tilde{u}_{s}=\sum_{i \in I_{\mathrm{C}}} u_{s} f_{i}
$$

where $\Upsilon_{j}$ is the asymptotic approximation of $\nabla \Phi_{j}$, the chemotactic potential of the chemoattractant $j$. We note that, due to the scale separations induced by the spatial rescaling, the 2D Poisson equation reduces to a monodimensional Poisson equation that can be integrated explicitly. The term $\Upsilon_{j}$ can be computed from the volume fractions $f_{j}$ or the chemo-attractant concentration $c_{j}$ with the following formula

$$
\begin{aligned}
& \Upsilon_{j}(r, z)=\frac{r}{R^{2}} \int_{0}^{R} s f_{j}(s, z) d s-\frac{1}{r} \int_{0}^{r} s f_{j}(s, z) d s, \quad j \in\{m, p o l\}, \\
& \Upsilon_{j}(r, z)=\frac{r}{R^{2}} \int_{0}^{R} s c_{j}(s, z) d s-\frac{1}{r} \int_{0}^{r} s c_{j}(s, z) d s, \quad j \in\left\{m o n, l a, H_{2}\right\} .
\end{aligned}
$$

The mixture velocity $u_{s}=\left(u_{s, r}, u_{s, z}\right)$, solution of the asymptotic version of the Stokes equation, is given by the explicit formulas

$$
\begin{aligned}
& u_{s, z}(r, z)=-\frac{\Lambda(r, z)}{\kappa(z)}\left(R \int_{0}^{z} \sum_{i \in I_{\mathrm{C}}} \gamma_{f_{i}}(R, y) d y-R^{2} U_{z, i n}+\frac{R^{2}}{2} U_{p e r}(z)\right)+U_{p e r}(z) \\
& u_{s, r}(r, z)=\left(-\frac{1}{r} \int_{0}^{r} s \partial_{z} u_{s, z}(s, z) d s-\sum_{i \in I_{\mathrm{C}}} f_{i}(r, z) \sum_{j \in I_{\mathrm{C}} \cup I_{\mathrm{S}}} \lambda_{i, j} \Upsilon_{j}(r, z)\right),
\end{aligned}
$$


where

$\Lambda(r, z)=\int_{r}^{R} \frac{s}{\mu(s, z)} d s, \quad \kappa(z)=\int_{0}^{R} s \Lambda(s, z) d s, \quad U_{z, i n}=\frac{1}{R^{2}} \int_{0}^{R} s \sum_{i \in I_{\mathrm{C}}} \gamma_{f_{i}}(s, 0) d s$.

We point out that the velocity field keeps track of the key parameters of the fluid mechanics: the heterogeneity of the viscosity $\mu$, the boundary conditions $\gamma_{f_{i}}(R, z)$ through the mucosa, the average intake $U_{z, i n}$, the peristalsis $U_{p e r}$, and the bacterial radial swim through the term $\Upsilon$. We also note that, taking $u=u_{s}$ and $\nabla \Phi=\Upsilon$, the volume conservation constraint (7) is preserved by construction, avoiding numerical problems of mass conservation. This approximate model represents a huge reduction of the computational load, with a speed up of about 70 , but gives accurate approximations of the initial model.

\subsection{Numerical implementation}

We solve Eqs. 21)-22 by a first-order time splitting method, coded in Matlab (MathWorks, version R2016b) and run on a linux architecture. The code sources can be found at https://forgemia.inra.fr/simon.labarthe/ gut-microbiota.git. At each iteration, we use a finite volume scheme on a MAC grid, with explicit time integration for the transport term (enforcing the positivity of the solution with a CFL condition) and implicit scheme for the diffusion. The spatial operators are applied alternatively in each spatial direction, which reduces the size of the linear systems to be solved. We end the time loop by integrating the source term with a semi-implicit Euler method that preserves the positivity. Namely, the negative contributions of the source function are passed on the left hand side and solved semi-implicitly, while the positive contributions are kept in the right-hand side and treated explicitly [31. The implicitation of the negative term does not involve any linear system inversion: due to the multilinear form of the different terms of the source function, the matrix to be inverted is diagonal.

We note that we take advantage of the equation $\sum_{i \in I_{\mathrm{C}}} f_{i}=1$ to avoid solving the equation on $f_{l}$ by taking $f_{l}=1-\sum_{i \in I_{\mathrm{C}}, i \neq l} f_{i}$. The model parameters can be found in Table Appendix A.4 for the parameters related to diffusion, speed and initial conditions, in Table Appendix A.5 for the parameters of the source function and in Table Appendix A.6 for the boundary conditions.

\subsection{Strategy of the numerical experiments.}

To colonize the colon, the microbial populations have to face the flow of the intestinal content. Several mechanisms have been identified as possible drivers of the microbial populations spatial distribution 8 such as 1) the polysaccharide level shaping the overall microbial population 2) the mucus zone providing nutrients and protecting the microbial populations from the luminal flow, 3) bacterial active swimming possibly favoring bacterial persistence, 4) epithelial motility, through peristalsis or segmentation contraction, slowing down the flow and helping maintaining the microbes in the colon. 


\section{Numerical experiments}

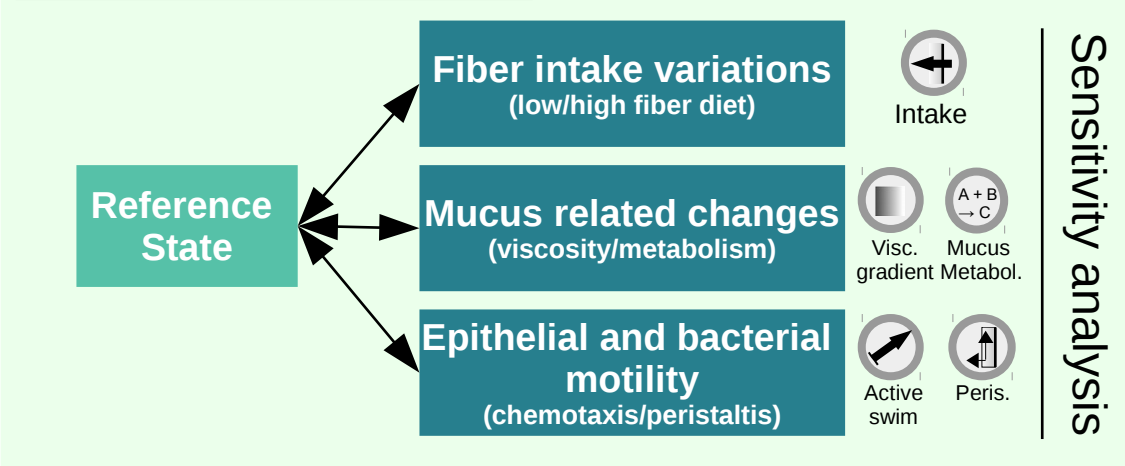

Figure 2: Outline of the numerical experiments. A reference state is defined in Section 2.4 and analysed in Section 3.1. We then study the contribution of diverse mechanisms by direct comparison with the reference state, as described in Section 2.4 The impact of dietary fiber levels is investigated in Section 3.2.1. In Section 3.2.2 we focus on the mucus-bacteria interactions. In Section 3.2.3 we study the outcome of the gut epithelium and bacterial motility. Finally, we explore the multifactorial processes involved in the spatial structure of the gut by providing a sensitivity analysis as described in Section 2.5 whose results are provided in Section 3.2 .4

Reference state.. In order to analyze the different mechanisms, we first define a basal reference condition for comparison: we knock down the peristalsis and the chemotactic activity, and select a polysaccharide input $\left(20\right.$ g.day $\left.^{-1}\right)$ representative of a normal reference diet. We then perform a long time simulation, starting from the mucus volume fraction equal to $f_{m}^{\text {init }}$, the liquid volume fraction equal to $f_{l}^{\text {init }}=1-f_{m}^{i n i t}$ and the other volume fractions $f_{i}^{i n i t}=0$, for all $i \in I_{\mathrm{C}}$, $i \neq l, m$. The mucus initial condition $f_{m}^{i n i t}$ is given by a sigmoid function, following [14, that distributes the mucus level from $f_{m, \text { min }}^{\text {init }}=0$ in the lumen to $f_{m, \max }^{\text {init }}$, the physiological amount of mucins in the mucus layer. We set

$$
f_{m}(t=0, r, z)=f_{m}^{i n i t}(r)=f_{m, \text { max }}^{i n i t}+\left(f_{m, \text { max }}^{i n i t}-f_{m, \text { min }}^{i n i t}\right) \frac{r^{\alpha_{m}^{i n i t}}}{r_{m}^{\alpha_{m}^{i n i t}}+r_{m}^{\alpha_{m}^{i n i t}}}
$$

where $r_{m}$ is a threshold defining the average thickness of the mucus layer and $\alpha_{m}^{i n i t}$ is a parameter which shapes the transition. This simulation is conducted until steady state, that is further used as a reference state. We check that this reference state can be taken as a proxy of a healthy gut microbiota, by verifying that key markers are recovered in a physiological range (see the Results section).

Assessing the impact of mechanisms.. We check the impact of the four putative mechanisms separately, by modifying only the model parameter that corresponds to the given mechanism. The reference state is taken as the initial condition of the additional numerical experiments, that are conducted until a new steady state is reached. This final state is compared to the reference initial state to assess the outcome of the experiment. The effect of dietary fiber is assessed by increasing or decreasing the fiber intake by $30 \%$. The effect of the mucus zone is checked in two different ways: in order to test if the nutrients provided 
by the mucus layer strongly shape the microbiota, we first knock down the mucus metabolism in the $\mathrm{B}_{\text {mon }}$ population by setting the consumption rate parameter to zero. As the mucus layer strongly impacts the local rheology, we next remove the viscosity heterogeneity by taking a homogeneous viscosity map $\mu=35 \times 10^{3}$ g.cm ${ }^{-1}$.day ${ }^{-1}$, in order to check if the rheology heterogeneities in the colon have an effect on the spatial repartition. The chemotactic function is introduced by setting $\lambda=1 / \operatorname{Grad}_{\max , j} \mathrm{~cm}^{2}$.day ${ }^{-1}$ where $\operatorname{Grad}_{\max , j}$ is the maximal value of the gradient of the chemotactic potential of the chemoattractant $j$ in the reference simulation, so that the characteristic value of the chemotactic speed is 1 , which is a small value with respect to the characteristic value of the longitudinal speed. The peristalsis is checked by setting $U_{p e r, r}=0$ and $U_{\text {per }, z}=-10$ for $5<z<155 \mathrm{~cm}$. The peristalsis is turned off near the boundaries $z=0$ and $z=L$ in order to preserve the consistency of the boundary conditions.

The other parameters used in the simulations can be found in Tables Appendix A.4. Appendix A.5 and Appendix A.6. The overall strategy is summarized in Fig. 2

\subsection{Sensitivity analysis.}

We perform a sensitivity analysis of the model outputs to parameter variations around the parameters identified in the previous simulations. We aim at testing the impact of selected parameters on the bacterial distribution. Namely, we select the epithelial motility ( $U_{p e r, z}$ parameter $)$, the bacterial chemotaxis $\left(\lambda_{i j}\right.$ parameters, that are modified in the same proportion), the viscosity gradient $\left(\mu_{\max , m}\right.$ and $\mu_{\max , l}$ parameters, that are shifted together $)$ and the fiber intake $\left(f_{\text {pol,in }}\right.$ parameter). We study the variations of the output $B(z)=\sum_{i \in I_{\mathrm{B}}} \frac{2}{R^{2}} \int_{0}^{R} r c_{i}(r, z) \mathrm{d} r$ when varying the selected parameters. We build a total factorial design by allowing for each parameter $\theta$ eight levels corresponding to a modification (in percentage) of $-50+i *(1 / 7) * 100, i \in[0,7]$ of its nominal value $\theta_{0}$ introduced in Tables Appendix A.4, Appendix A.5 and Appendix A.6. Testing 4 parameters results in a design containing 4096 different sets of parameter values and the same number of model runs to perform the sensitivity analysis. The model outputs are post-processed with the $\mathrm{R}$ package Multisensi (https://CRAN.Rproject.org/package=multisensi). We compute with Multisensi the descriptive statistics on $B(z)$, the distribution of the first order Sobol index of each parameter along the colon length with the method introduced in [23], see Fig 10. We recall that the first order Sobol index $S_{\theta}(z)$ of a given parameter $\theta$, for a given $z \in(0, L)$ reads

$$
S_{\theta}(z):=\frac{\operatorname{Var}(\mathbb{E}(B(z) \mid \theta))}{\operatorname{Var}(B(z))} .
$$

Furthermore, we compute the PRCC (Partial Rank Correlation Coefficient) of $B(z)$ relatively to the 4 parameters, using the same total factorial design and the $\mathrm{R}$ package Sensitivity (https://cran.r-project.org/web/packages/sensitivity/index.html). A $95 \%$ confidence interval for the PRCC indices is computed with 100 bootstrap runs. 


\section{Results}

We first check that the reference state reproduces the results introduced in 29 and 28. This numerical experiment is subsequently used as a reference control to assess the effect of different mechanisms on the spatial structure of the colonic content: diet variations (subsection 3.2.1), viscosity gradient and mucus metabolism (subsection 3.2.2), peristalsis and active motion (subsection 3.2.3). We finally compare the relative influence of each mechanism in the multifactorial process leading to the spatial structuration of the large intestine (subsection 3.2.4 through a sensitivity analysis of the model.

\subsection{Characterization of the reference state}

We aim at studying the mechanisms that drive the spatial structure of the gut microbiota at equilibrium. We thus need to characterize a correct proxy of the homoeostatic state of the gut microbiota. Starting from a colon with liquid and mucus only and setting a normal microbial and metabolite influx, we perform a long term simulation of our PDE model until steady state, defined as the reference state. We note that the motile activity of the epithelial wall and the bacterial active swimming are turned-off in this reference state (i.e. $U_{\text {per }}=(0,0,0)$ and $\lambda_{i j}=0$ for all $\left.i \in I_{\mathrm{B}}, j \in I_{\mathrm{S}} \cup\{m, p o l\}\right)$ to allow comparison with previous studies [29, 8, and to provide a negative control for these effects. We first assess that this equilibrium correctly reproduces physiological markers of the structure and functions of a healthy microbiota in the colon.

\subsubsection{Longitudinal structure of the colon in the reference state.}

The longitudinal distribution of several parameters driving the fluid mechanics and the overall microbial steady-state levels are displayed in Fig. 3. We can see (Fig 3 A, blue line) that the averaged longitudinal speed decreases strongly in the proximal and transverse sections, where the pumping activity by the mucosa is maximal (yellow curve, Fig 3-A). It then reaches an average speed of $4.30 \mathrm{~cm} \mathrm{~d}^{-1}$, which corresponds to an outgoing flux of $169 \mathrm{~mL} \mathrm{~d}^{-1}$, in the range of natural water excretion in feces $\left(100-200 \mathrm{~mL} \mathrm{~d}^{-1}\right.$ [18]). The radial speed reaches its highest level at the beginning of the colon and then drops off in the distal part to negligible values. The radial transport is thus expected to dominate over the radial diffusive process in the proximal colon, while the dominance ratio is reversed distally.

A key parameter for the speed dynamics is the viscosity distribution. We can observe (green curve, Fig 3-B) that the viscosity of the colonic mixture increases all along the colon until reaching a maximal value in its distal part before a slight decrease, due to mucus consumption by the microbiota. The viscosity increase reflects water absorption and the resulting concentration of the other mixture components. The microbial activity (red curve, Fig 3-B), defined as the sum over bacteria of the growth and death rates, is mostly driven by the polysaccharides metabolism. The fibers start accumulating in the proximal colon, under the effect of a strong water pumping in this compartment (blue curve, Fig 3-B). They are next entirely consumed by the microbiota in the transverse colon, consequently increasing the microbial activity that reaches its maximum value in the early distal compartment. Then, the microbial metabolic activity drops off until a plateau phase that corresponds to the mucus degradation only. The microbial density (magenta curve, Fig 3-B), first distributed exponentially 

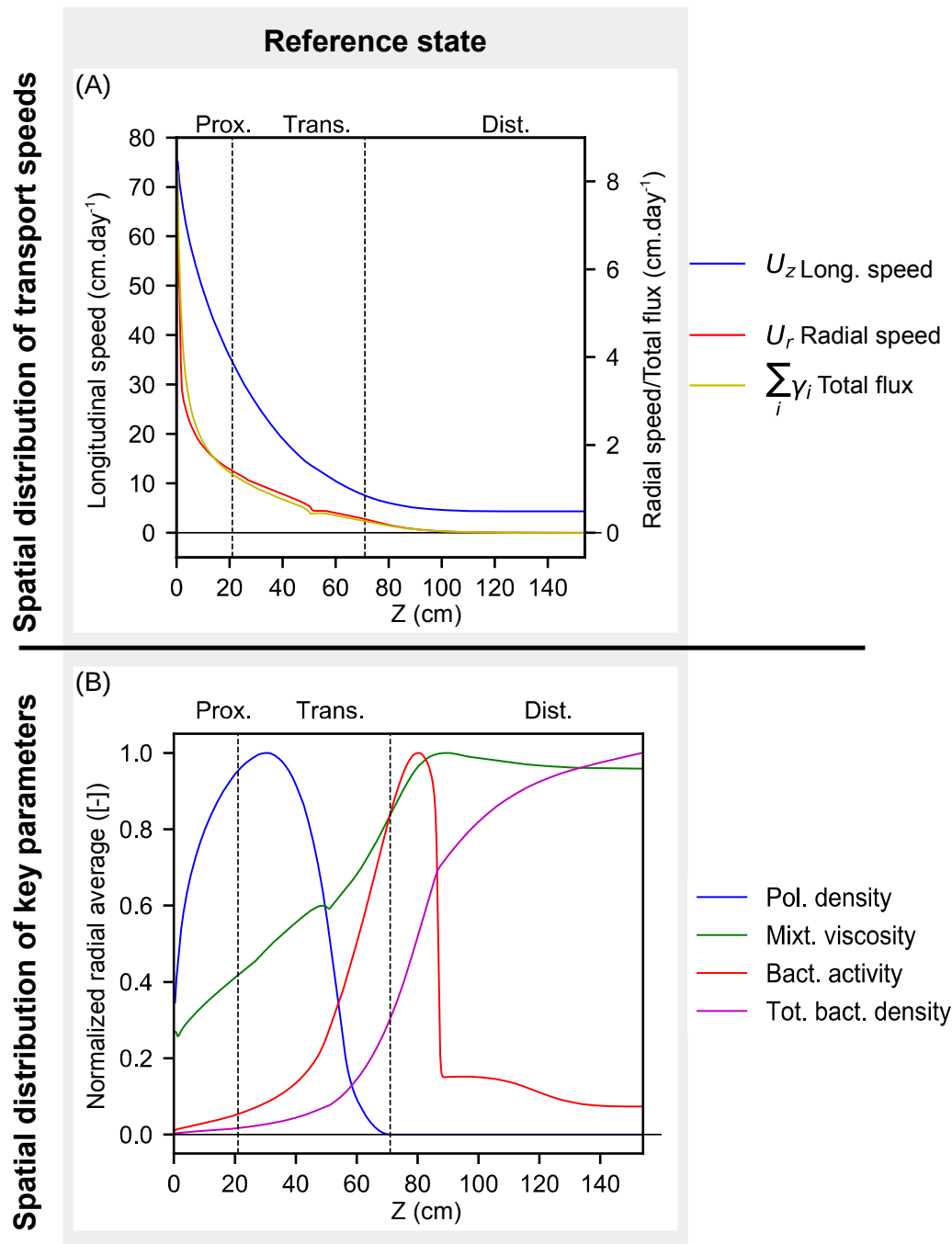

Figure 3: Transport speeds and key parameters of the spatial structure of the reference state (see Section 3.1). (A) The longitudinal distribution of the radially averaged radial $\left(U_{r}(z):=\frac{2}{R^{2}} \int_{0}^{R} r u_{s, r}(r, z) d r\right)$ and longitudinal speeds $\left(U_{z}(z):=\frac{2}{R^{2}} \int_{0}^{R} r u_{s, z}(r, z) d r\right)$, together with the total flux of water and mucus through the mucosa $\left(\sum_{i} \gamma_{i}(z)\right)$, are displayed. We indicate by vertical dashed lines the limits of the colon compartments that are considered for observation issues: proximal, transverse and distal colon. (B) In the lower panel, the average values of key parameters along the colon are presented. The different quantities are normalized by their maximal value to allow representation in the same graph. The maximal values of polysaccharide density, mixture viscosity, microbial functional activity and total microbial density are respectively $6.74 \cdot 10^{-2}, 3.88 \cdot 10^{3} \mathrm{~g} \mathrm{~cm}^{-1} \mathrm{~d}^{-1}, 8.34 \cdot 10^{-3} \mathrm{~d}^{-1}$ and $6.10 \cdot 10^{-2}$.

in the first part of the colon, displays a slope break in the distal part reflecting the metabolic switch from dietary fibers to mucus. The total bacterial volume fraction at the colon exit is $6 \cdot 10^{-2}$, which corresponds to a bacterial density of $0.6 \cdot 10^{11} \mathrm{CFU} \mathrm{g}^{-1}$ of feces, within the range of observed data 36. Furthermore, the total mass of the gut microbiota is $85 \mathrm{~g}$ and the total number of bacteria is 
$8.5 \cdot 10^{13}$, which correspond respectively to half and twice the measured bacterial levels reported in [36].

To sum up, the reference state that was designed reproduces macroscopic features of a healthy colon microbiota, while giving insights on the spatial distribution of observables such as bacterial levels, colon content viscosity, transit speed, fiber consumption or epithelial fluxes. We now further study the spatial distribution of bacterial levels and SCFA production, in order to compare the reference model with existing studies [29].

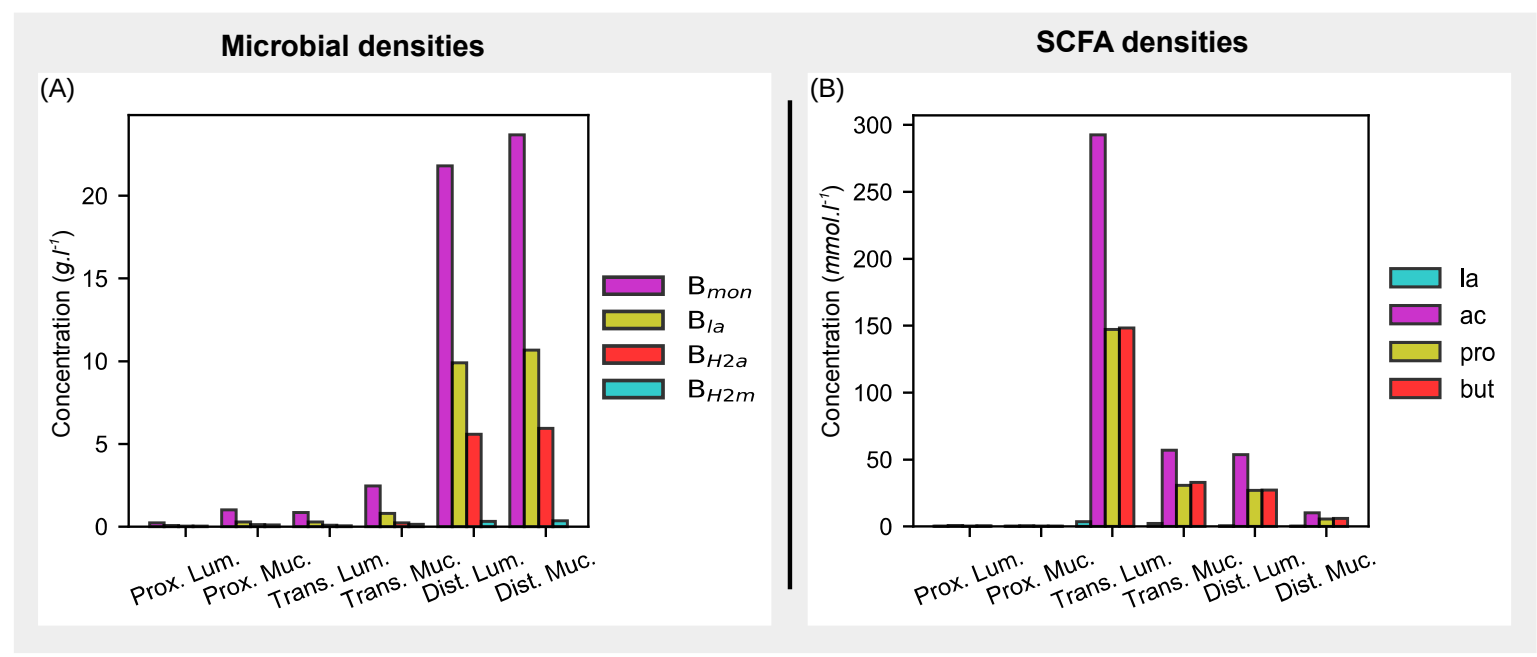

Figure 4: Microbial and SCFA levels in the different compartments in the reference state (see Section 3.1.2). We display bar plots of the microbial population (A) and SCFA (B) averaged densities in 6 different compartments of the large intestine, formed by the luminal and mucosal regions in the proximal, transverse and distal colon. The mucosal area is defined by the points located at less than $0.2 \mathrm{~cm}$ from the mucosal boundary.

\subsubsection{Spatial distribution of the microbiota and SCFA in the reference state.}

The averaged values presented in Fig 3 provide an accurate view of the longitudinal distribution but do not render the radial repartition of the colon content. We then display the distributions in six compartments formed by the luminal and mucosal parts of the proximal, transverse and distal colon (Fig 4 ). We can observe that the microbial population levels are higher in the mucosal part of the proximal and transverse compartments, but lower for the SCFAs. This is mainly the result of the important water absorption in these compartments, which tends to accumulate the mixture components near the mucosa, including the bacteria. On the contrary, the absorption of SCFAs by the mucosa lowers the fatty acids levels in the mucosal part. In the distal part, where the mixture diffusion and the radial speed balance, the microbial distribution is much more homogeneous. Due to absorption, the SCFAs are still depleted distally near the mucosa.

The microbial levels reflect the trophic interactions: the top bacteria in the trophic chain, i.e. the poly/monosaccharides consumers $\mathrm{B}_{m o n}$, are also the most present in each compartment. Their level reaches approximatively twice the level 
of the lactate consumers $\mathrm{B}_{l a}$, which in turn is greater than the acetate producers $\mathrm{B}_{H_{2}, a}$ (Fig 3). The methanogens $\mathrm{B}_{H_{2}, m}$, which are repressed by the increasing $\mathrm{pH}$ along the colon, present smaller levels. The complete distribution maps (Fig. Appendix B.1, supplementary materials) show that there is a correspondence between these trophic interactions and the spatial distributions: the higher is the bacterial position in the trophic chain, the more proximal is the population repartition front.

The millimolar ratio Acetate:Propionate:Butyrate in the luminal compartments are 82:41:43 in the proximal part, 74:37:37 in the transverse segment and 57:28:29 in the distal colon, in agreement with the predicted values in [29] and with the experimental measurements in [39, 11. The overall levels of SCFA in the transverse lumen are over-estimated by our model compared to experimental post mortem measures in this compartment (560 vs $118 \mathrm{mM})$. However, in the mucosal transverse (121 predicted vs $105 \mathrm{mM}$ measured) and in the distal compartments (103 vs between 72.4 and $87.5 \mathrm{mM}$ measured), the model is in good agreement with experimental data [39, 11].

Having checked that the spatial distribution of the bacterial levels and the SCFA concentrations of the reference state are in good agreement with modeling [29] and experimental results [39, 11, we can now take advantage of the spatial resolution of our model to study a key element of the colon ecology: the mucus layer.

\subsubsection{Mucus layer}

The mucus layer is an interface between the microbiota and the colon epithelium, playing a key role in the symbiotic interactions between the host and the commensals. It is a passive protection against microbial invasion, but it also provides nutrients and a protective environment against the luminal flow for the bacteria. 20.

In our model, the mucus is represented by the mucin density, which impacts the mixture viscosity through the mucus viscosity function $\mu_{m}$. Indeed, as the sigmoidal function $\mu_{m}$ is very stiff (see Eq. (16) ), the viscosity threshold $f_{m, t h r}=0.0425$ represents the limit of the mucus layer. When the mucus density is above this threshold, we will consider that the corresponding spatial point is inside the mucus layer. At steady state, the mucus layer has a minimal thickness of about $1.7 \mathrm{~mm}$ in the proximal colon (Fig $5 \mathrm{~B}$ ), where the microbial populations are small and the radial speed is high due to water absorption. In the transverse colon, the mucus layer is thicker ( $4.3 \mathrm{~mm}$ as maximum) due to the diffusive process that counterbalances the radial transport in that region. In the distal part, the mucus layer is consumed by the microbial populations, after the integral consumption of the fibers, and get thinner again until reaching $2.4 \mathrm{~mm}$. Human data for the mucus thickness are currently lacking in the bibliography [19]. In rats, the total mucus layer thickness (including the firmly and loosely adherent layers) are respectively 0.480 and $0.829 \mathrm{~mm}$ at the end of the ileum and in the colon [1]. The colon radius in rats is $0.4 \mathrm{~cm}$ [25]. Assuming that the rheologic and hydrodynamic parameters are the same in rats, a simple rescaling of our model outcome would give a maximal mucus thickness of $0.272,0.688$ and $0.384 \mathrm{~mm}$ in the proximal, transverse and distal colon, which is comparable to the rat data. We also observe (Fig. Appendix B.1, supplementary materials) that the bacterial populations are higher in the vicinity of the mucus layer and 

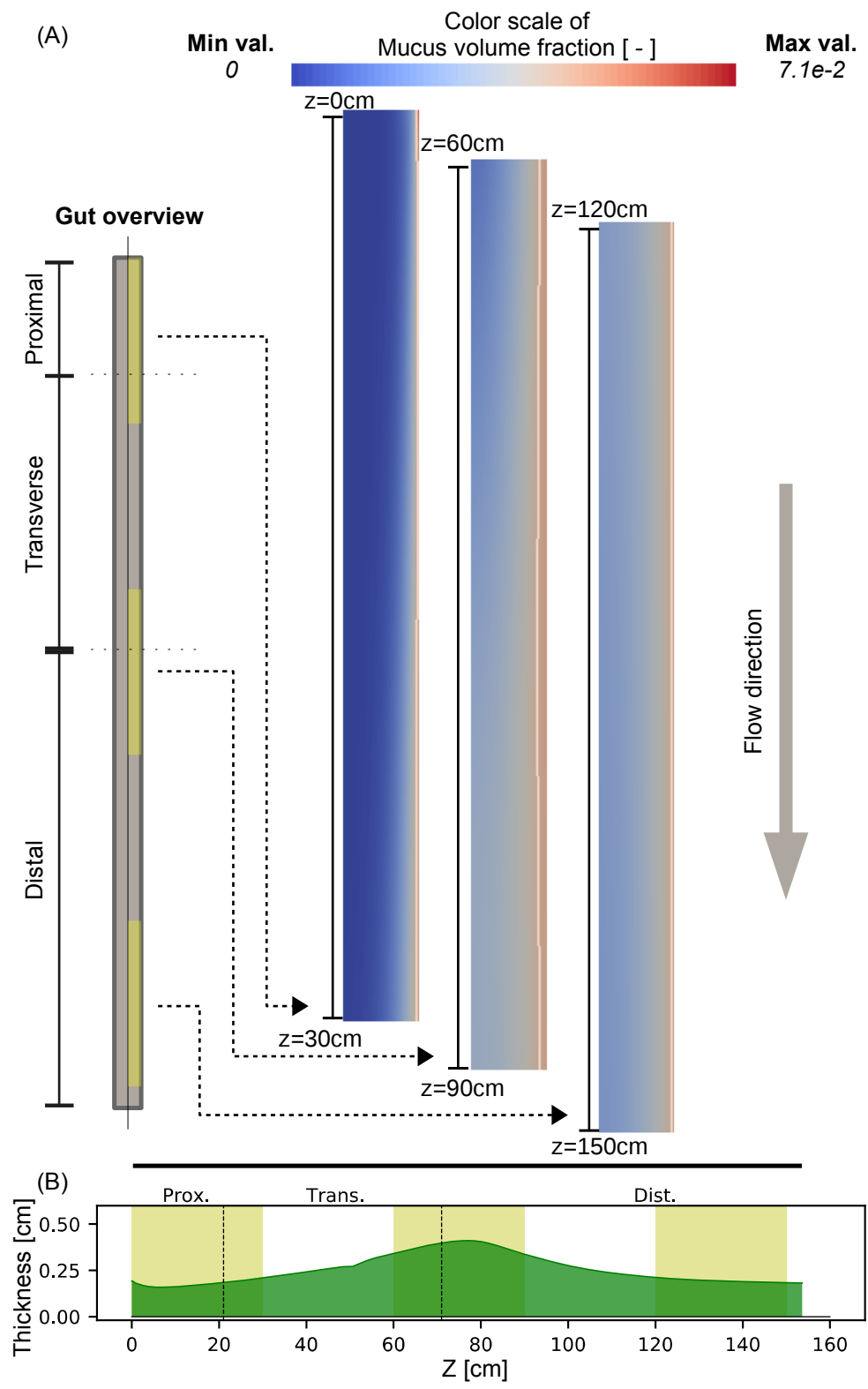

Figure 5: Spatial distribution of the mucus in the colon (see Section 3.1.3). The steady-state mucus distribution is displayed in 3 different regions of the proximal, transverse and distal parts (A, top) of the large intestine together with the isoline defined by the mucus volume fraction $m=0.0425$ which represents in our model the limit of the mucus layer: we represent longitudinal sections (for $r \geq 0$ ) of the cylindrical colon; the axis of the cylinder is the left boundary of the images while the right boundary is the mucosa; the upper part of the images is the most proximal and the digestive flux is directed from the top to the bottom of the figure. We then display the mucus layer thickness (B, bottom, green plot). The mucus layer is thin in the proximal colon and get thicker in the transverse part, to be reduced again in the distal bowel. The yellow zones that are displayed in the schematic view of the colon and in the mucus layer plot indicate the domains covered by the proximal, transverse and distal plots of A. 
lower in the lumen, reproducing a phenomenon recently observed in vivo by fluorescence imaging with labelled microbial strains 43 .

Hence, our model reproduces structural features of the mucus layer physiology, but also ecological aspects, such as the favorable zone for the commensals in the vicinity of the mucus. We now go deeper in the deciphering of the drivers of the spatial structure of the colon environment.

\subsection{Study of the drivers of the gut microbiota spatial structure}

The reference state is perturbed by modifying a single mechanism included in the model. The consecutive steady state is compared to the reference to assess the importance of the corresponding parameter in the homeostatic regulations. To check the significance of the observed variations, we first check that the reference state is robust respectively to the initial condition: when the initial condition is chosen randomly, the resulting steady-state is quasi-identical to the reference state (relative difference less than $0.1 \%$ in $L^{\infty}$ norm).

\subsubsection{Spatial perturbations induced by diet variations.}

The amount of fiber in the diet is known to be directly linked to the overall microbial and SCFA densities in the colon. We use the model to provide a more accurate insight on the spatial impact of high and low-fiber diets on the colon environment (see Section 2.1.5, §Mass transfers through the boundaries Dietary inflow on $\Gamma_{i n}$ ) by respectively decreasing or increasing the averaged polysaccharide daily input by $30 \%$. We plot in Fig. 6 the speed variations induced by the diet changes, and the modification of the key parameters that were defined in Fig. 3. We observe (Fig 6. A-B ) that the longitudinal speed slightly decreases or increases with the fiber intake, which is consistent with the fact that more fibers shorten the transit time in the colon. In our model, these spatial variations are directly related to the modeling of water absorption: in the high-fiber diet, more fibers accumulate near the epithelial wall, leading to less water available for absorption. This enhances the water density in the lumen and consequently, the chyme fluidity and the transit.

The differences in fiber intake impact the fiber distribution in the proximal colon (blue curves, Fig 6. C-D). The fiber concentration increases with the fiber intake, and the fiber distribution is spread out when more fibers are ingested. This is reflected in the microbial activity distribution (Fig Appendix B.3. A-B, supplementary material), which presents a shift of the peak activity towards the distal part for higher fiber diets. The microbial densities (magenta curves, Fig 6 C-D) in turn reflect these spatial variations of microbial activity: the microbial density is first slightly higher in the transverse colon for low-fiber diet, but the tendency is rapidly reversed from the beginning of the distal bowel. The overall microbial density is higher for higher-fiber diets, as expected. Conversely, the viscosity distribution (green curves, Fig 6 , C-D) is only slightly modified by the diet variations.

Differences in microbial and SCFA densities can also be observed in all the colon compartments (Fig. Appendix B.2, supplementary data). The microbial and SCFA levels are directly linked to the quantity of dietary fibers: highfiber diets enhance the gut microbiota function by increasing the SCFA levels, in accordance with experimental studies [11. The microbial levels are first equivalent for all diets in the proximal regions but noticeable differences are observed in the distal parts. 


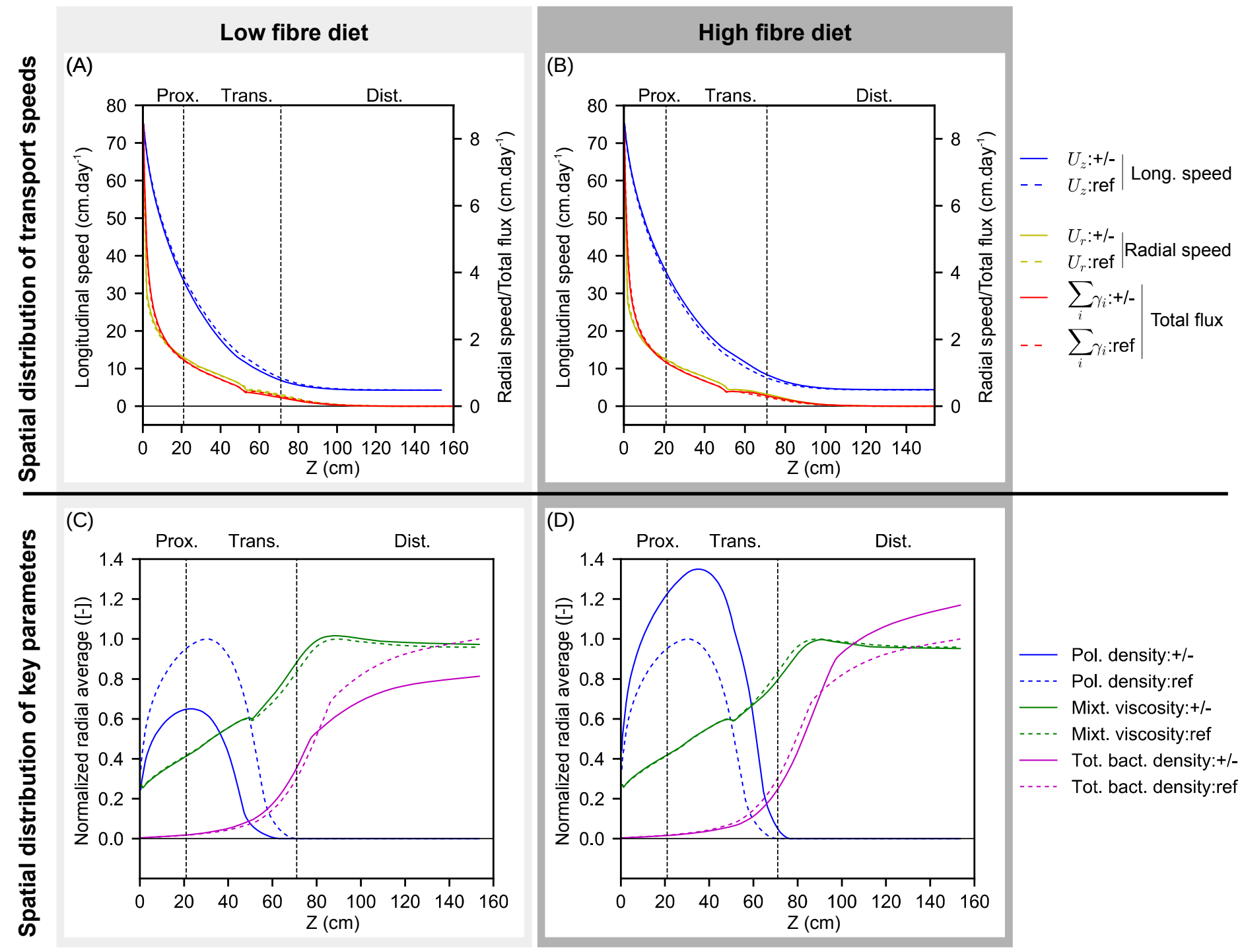

Figure 6: Impact of fiber diet on the transport speeds and on the spatial structure. (See Section 3.2.1) We reproduce the quantities of Fig. 3 with a low-fiber diet (30\% decrease of polysaccharide input, A and C), or a high-fiber diet ( $30 \%$ increase of polysaccharide input, B and $\mathrm{D})$, that we compare with the reference fiber diet (same polysaccharide input, dashed lines). In the upper panels (A-B), the speed distribution is reproduced, whereas the lower panels (C-D) display the spatial distribution of relevant parameters: all the values are normalized respectively to the maximal values of the reference diet (see Fig 3 for nominal values). Higher fiber diet increases the transit speed, the fiber concentration and the microbial levels, while slightly locally decreasing the viscosity. Lower fiber diet has a reverse effect.

The fiber intake modulates the spatial distribution of the bacterial activity and of the bacterial levels: more fibers change the hydrodynamics in the proximal gut, increasing the transit speed, which shifts the bacterial plateau distally, but 
supports the bacterial growth by providing more substrates. We now focus on the interactions between the bacteria and the mucus, which are twofold in our model: 1) a direct interaction through mucus metabolism by the $\mathrm{B}_{\text {mon }}$ populations, 2) an indirect interaction through the mucus-dependant rheology, which modifies the fluid dynamics and thus the bacterial growth.

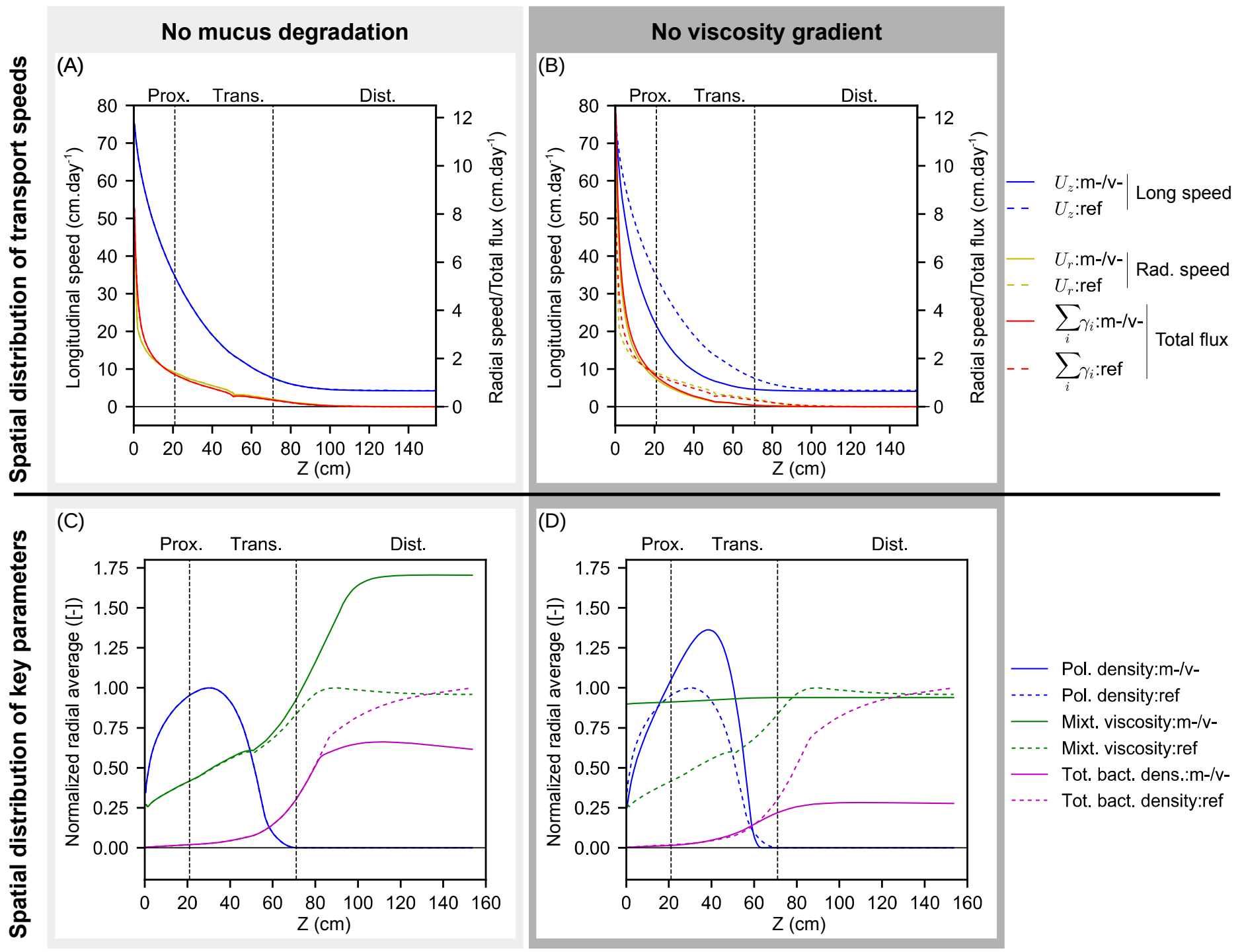

Figure 7: Impact of viscosity and mucus degradation on the outcome of the model (See Section 3.2.2). We reproduce some quantities of Fig. 3 with no mucus degradation $(m-$, $\mathrm{A}$ and $\mathrm{C}$ ) or with a homogeneous viscosity $(v-, \mathrm{B}$ and $\mathrm{D})$, compared with the reference-fiber diet of Fig. 3 (ref, dashed lines). All the values are normalized respectively to the maximal values of the reference. The mucus degradation has an effect in the distal bowel only. A homogeneous viscosity has a deep impact on the bacterial activity and the microbial levels. 


\subsubsection{Microbes-mucus interactions: mucus-induced viscosity gradients promote the bacterial growth}

Due to the ecological importance of the mucus layer (see Section 3.1.3), we study the mechanisms that drive microbes-mucus interactions in the model. In our model, the nutritional interactions between the host and the commensals through the mucus composition and its host-derived polysaccharides are taken into account by the bacterial metabolism (see Section 2.1.4, §Microbial metabolic activity). In the mean time, the protective action for the bacteria of the mucus layer against the luminal flow is provided by the mucus-dependant viscosity (see Section 2.1.5, §Viscosity heterogeneities). To investigate both mechanisms, we perform a simulation without viscosity gradient by setting a homogeneous mucus function with a median value of $35.10^{3} \mathrm{~g} \mathrm{~cm}^{-1} \mathrm{~d}^{-1}$, and a simulation without mucus metabolism by the $\mathrm{B}_{\text {mon }}$ population, that we compare with the reference simulation (Fig. 7). As expected, the knock out of the mucus metabolism only very slightly alters the speed fields (Fig. 7 A, where the curves are superimposed with the reference state). But the longitudinal speed is significantly reduced due to a higher water absorption in the proximal colon, when the viscosity is homogeneous (blue curve, Fig. 7 B).

A reduced longitudinal speed, by enhancing the retention time, usually promotes bacterial growth. However, the bacterial activity and the overall bacterial populations are drastically reduced (a $60 \%$ decrease comparatively to the reference) in the absence of viscosity heterogeneities (Fig. Appendix B.3 D (supplementary material), and magenta curves, Fig. 7, D). These behaviors can be explained by a viscosity-dependant slowdown zone near the mucosa. When the viscosity is mixture-dependant, the mucus layer increases the viscosity gradients, which reduces the longitudinal speed near the mucosa. This deceleration, noticeably marked in the proximal part (dashed blue lines, Fig Appendix B.4. B, Supplementary Materials), enhances the local retention time and promotes matter accumulation near the mucus layer, reducing water absorption and increasing water availability for bacterial growth. When the viscosity is homogeneous, a reversed mechanism occurs, promoting water absorption, which results in a reduced bacterial growth due to volume saturation.

The suppression of the mucus degradation only slightly modifies the overall dynamics in the proximal part of the colon, but has a sensitive impact in the distal bowel (Fig. 7. C). In this portion of the digestive tract, there are no dietary fiber left, and if the microbial populations are not able to metabolize the host-derived polysaccharides, the bacterial mortality is the preponderant component of the microbial activity (Fig. Appendix B.3 C, supplementary material). The overall population levels are therefore reduced in the distal part compared to the reference model. However, they are still more than $50 \%$ higher than when there is no viscosity gradient (Fig. 7. C-D), suggesting that, in our model, the preponderant mucus-microbes interaction for bacterial growth is the local hydrodynamics near the mucosa induced by the rheology of the mucus layer.

The identification of the vicinity of the mucus layer as a slowdown zone favoring the bacterial growth is consistent with recent experiments that identified higher bacterial concentrations near the mucus layer in rodents 43. The model provides a possible mechanism for such an observation by identifying a viscositydependant slowdown zone near the mucosa. This slowdown zone leads to the 
radial distribution observed in Fig. Appendix B.1 (supplementary materials) where the bacterial populations grow proximally in the mucus vicinity, where the retention times are favorable, until reaching a good balance between bacterial levels, transit speed and growth rate to invade the luminal part. This observation is consistent with the mucus zone acting as a reservoir seeding the lumen 8 . But in vivo, more active processes occur at the same time, such as gut motility or bacterial active motion, that we now investigate.

\subsubsection{Strong effect of epithelial motility and bacterial active swimming on the spatial structure.}

The gut motility, involving segmentation contractions that mix the luminal content and peristaltic contractions that provide a longitudinal net motion, appears to be an important mechanism of the host epithelial physiology to regulate the luminal transit. Meanwhile, the bacteria can express flagella genes, providing motility to actively face the luminal flow. We then investigate the effect of epithelial motility and bacterial active swimming on the overall spatial structure of the colonic content (See Section 2.1.5. §Epithelial motility and peristalsis for the epithelial motility and Section 2.1.4. §Microbial active motion for the active swimming).

We reproduce the reference simulation with a constant peristaltic value of $U_{z}=-10 \mathrm{~cm} \mathrm{day}^{-1}$ for $5<z<155 \mathrm{~cm}$, representing the net effect of the peristaltic and segmentation contractions of the colon wall as a upstream flow near the mucosa 24. We next modify the reference simulation by endowing the microbial populations with slight active swimming capabilities (with characteristic chemotactic speed of $1 \mathrm{~cm}$ day $^{-1}$, several orders of magnitude under the longitudinal characteristic transit speed $\left(100 \mathrm{~cm} \mathrm{day}^{-1}\right)$ and the maximal speed observed for the bacterial swimmers, also about $100 \mathrm{~cm} \mathrm{day}^{-1}$ [8]). An asymptotic analysis of the different operators shows - see Material and Methods, section 2.2 that the active swimming in the longitudinal direction can be neglected: we then only consider the radial direction of the bacterial motility in the model. The microbial populations included in the model have therefore no possibility to swim upstream against the intestinal transit.

We can observe (blue curve, Fig 8,A) an important increase of the averaged longitudinal speed when the epithelial motility is active, which is counter-intuitive, since the peristaltic activity is applied in the upstream direction. This increase comes from a redistribution of the longitudinal speed along the colon radius. Upstream speeds are observed near the epithelial wall in the peristaltic case, versus null speeds for the reference and the chemotactic experiments, resulting in a large increase of the longitudinal speed in the luminal part to preserve the volume (see Fig. Appendix B.5A-B, Supplementary materials and its legend for details on volume conservation). The radial speed and the mucosal flux are rather similar to the reference state (yellow and red lines, Fig. 8, A) except on the peaks at $z=5 \mathrm{~cm}$ and $z=155 \mathrm{~cm}$ which correspond to velocity discontinuities at the limit of application of the peristaltic activity. Regarding the speeds components, the chemotactic activity of the bacterial has a very little impact (Fig 8,B and Fig. Appendix B.5,B-D).

As expected, the epithelial motility induces a strong upstream shift of the microbial populations, enhancing the bacterial functional activity in the upper transverse colon and promoting a fast consumption of the fibers in the proximal colon (magenta and blue curve, Fig 8, C and Fig. Appendix B.3, E, supplementary 


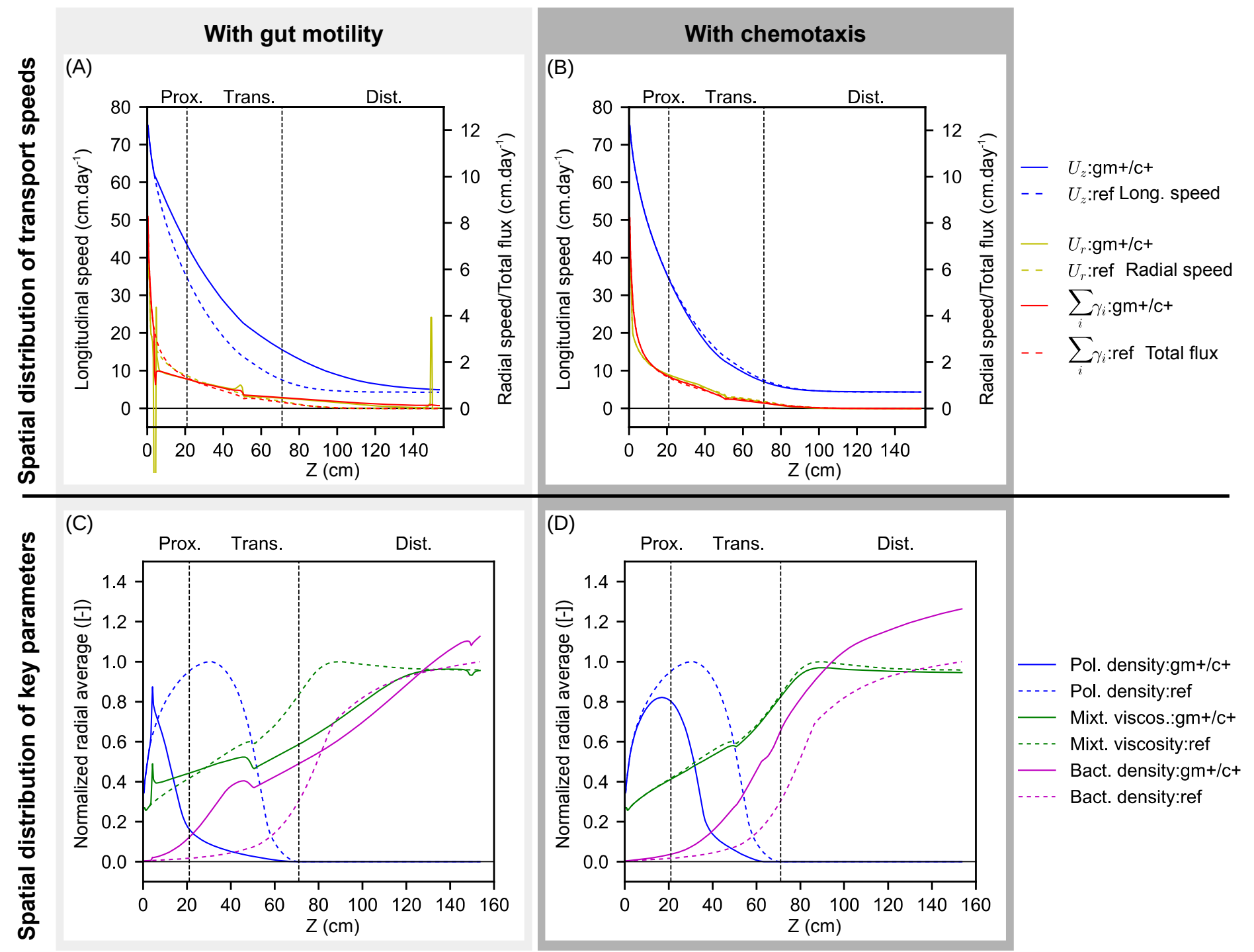

Figure 8: Impact of peristalsis and chemotaxis on the outcome of the model (See Section 3.2.3). We reproduce some quantities of Fig. 3 with epithelial motile activity $(g m+$, $\mathrm{A}$ and $\mathrm{C}$ ) or chemotactic active swimming $(c+, \mathrm{B}$ and $\mathrm{D})$, compared with the reference-fiber diet of Fig. 3 (ref, dashed lines). All the values are normalized respectively to the maximal values of the reference. The epithelial motility shifts proximally the bacterial activity, enhancing the bacterial levels in the proximal and transverse colon, while reducing the viscosity. The bacterial active motion promotes an earlier colonization of the colon, resulting in increasing proximally the metabolic activity and the microbial concentrations.

material). The bacterial active swimming also promotes the bacterial levels proximally but with smaller magnitude (magenta, red and blue curves, Fig 8,D). However, the bacterial metabolism is noticeably boosted in that case, speeding up the carbohydrate consumption, comparatively to the reference simulation 
(blue curves, Fig. 8.D and Fig. Appendix B.3, E, supplementary material). The viscosity (green curves, Fig 8,C-D) is strongly impacted by the epithelial motility, which is related to local modifications on the mucus distribution, but not by the bacterial active motion. We can observe than the wall motility has a focal impact on the overall microbial populations, with a massive increase in the proximal colon and a lower increase at the end of the distal intestine, whereas the enhancement of the bacterial levels is more regular along the colon after activation of the chemotactic capabilities (magenta curves, Fig 8,C-D). We emphasize that the motile speeds applied to the bacteria are very low: the maximal (in $z$ ) radial average of the chemotaxis speed are respectively $1.87,0.88$, 1.42 and $1.42 \mathrm{~cm} \mathrm{day}^{-1}$, which can be compared to observed swimming speeds in viscous media: for example, bacterial swimmers (Bacillus Thuringiensis) were observed swimming in biofilms with motile speeds ranging between 2 to 16 $\mu m . s^{-1}$, i.e. 17.3 to $138.2 \mathrm{~cm} \mathrm{day}^{-1}$ [17. A bacterial swimmer (Pseudomonas aeruginosa) was also tracked in vivo in a zebra fish gut swimming at speeds up to $24 \mu \mathrm{m} . \mathrm{s}^{-1}\left(207.6 \mathrm{~cm} \mathrm{day}^{-1}\right)$ in the luminal part of the large intestine 33 . Those observed speeds are up to two orders of magnitude over the motile speeds involved in our model. We also note that this speed is applied radially, toward the mucosa, with no longitudinal upstream swimming included in the model. These low radial speeds are sufficient to get this noticeable positive impact on the total microbial densities.

In conclusion, those host and bacteria-derived motilities improve the growth of the bacterial populations by reducing the transit time proximally (peristalsis) or by allowing the bacteria to quickly reach the favorable zone in the vicinity of the mucus layer (bacterial active motion). Metaproteomic studies showed that the expression of motility genes is low in commensal microbiota [42, but the model indicates that small motile capabilities (one to several orders of magnitude under observed active swimming speeds for bacteria in viscous media) are enough to enhance the bacterial levels.

\subsubsection{Deciphering the multifactorial process of spatial structure with sensitivity analysis}

We emphasized several mechanisms that impact the bacterial levels and the spatial distribution of the colon contents: fiber intake, viscosity gradients, epithelial and bacterial motility. We now study their relative influence in the multifactorial mechanisms involved in the large intestine spatial structure.

We first check that the previous mechanisms result in a positive outcome when combined, i.e., that the respective effects of chemotaxis, gut motility and viscosity gradients do not compensate each other resulting in a null net effect on the colonic content composition. We can observe (blue line, Fig. 9p that the polysaccharide density drops down very early in the colon, while the bacterial activity reaches its maximal value in the proximal colon. The viscosity is globally reduced, but conserves its increasing profile along the large intestine. The bacterial populations start growing at the beginning of the proximal colon, which is a strong improvement comparatively to the reference experiment where the microbial colonization was effective at the early distal colon only. At the end of the distal bowel, the overall bacterial levels are increased up to $50 \%$ compared to the reference.

A more accurate study is performed through a global sensitivity analysis of the different parameters. We shift conjointly the parameters defining the mixture 


\section{With gut motility, chemotaxis and viscosity gradients}

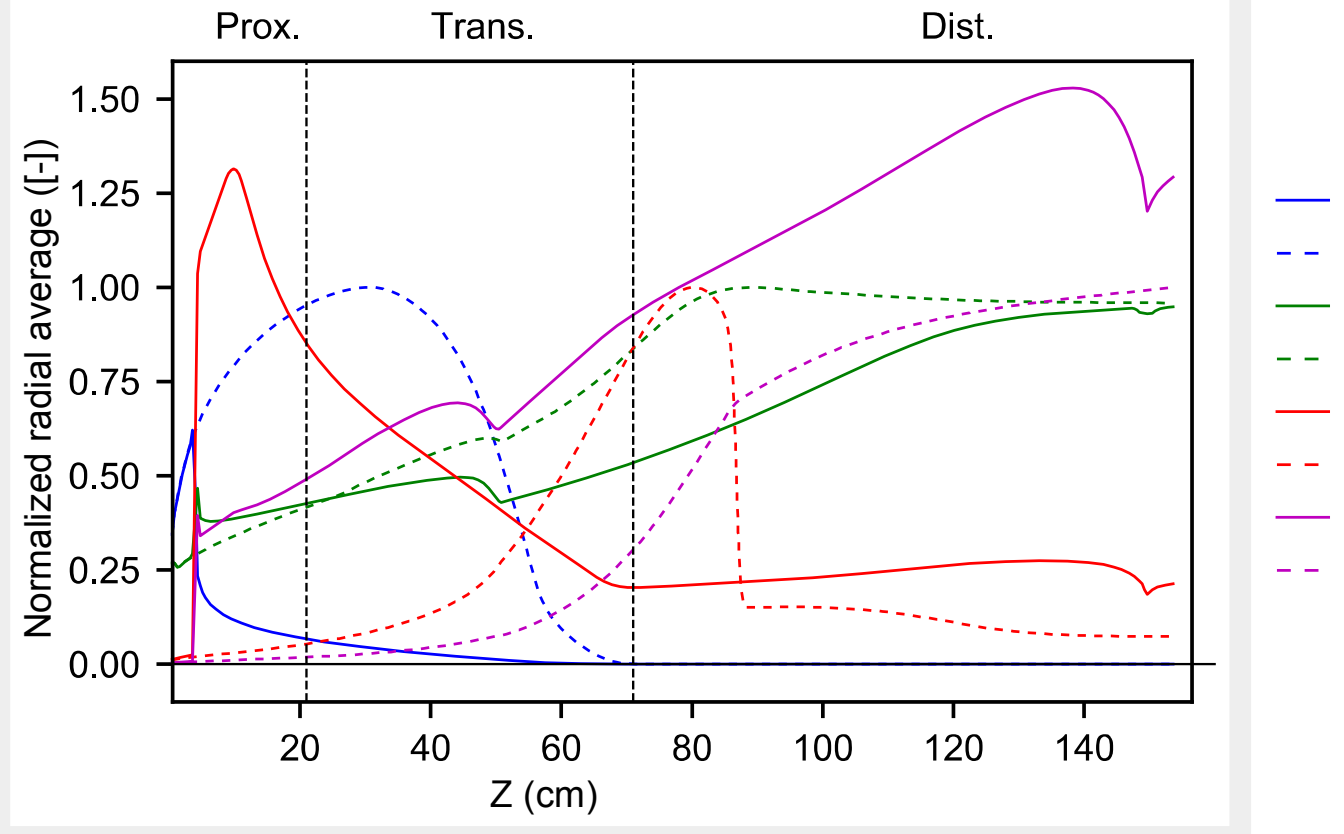

Pol. density:all

Pol. density:ref

Mixt. viscosity:all

Mixt. viscosity:ref

Bact. activity:all

Bact. activity:ref

Tot. bact. density:all

Tot. bact. density:ref

Figure 9: Longitudinal distribution of the averaged key parameters when all the mechanisms are combined (See Section 3.2.4). We investigate the impact of the combination of all the effects, i.e. peristalsis, chemotaxis and viscosity heterogeneities on the outcome of the model. We reproduce the quantities of Fig. 3 with all the mechanisms, i.e. peristalsis, viscosity gradients and chemotaxis (all, solid lines), compared with the reference-fiber diet of Fig. 3 (ref, dashed lines). All the values are normalized respectively to the maximal values of the reference. The metabolic activity and the bacterial levels are boosted when all the mechanisms are combined.

viscosity, the epithelial motility intensity, the bacterial swimming magnitude and the fiber input to assess their impact on the overall bacterial repartition along the colon. Namely, we study for each $z \in(0, L)$ the variations of the radially averaged total bacterial population $B(z)$ when varying the parameters (see the Material and Methods for a precise description of the sensitivity analysis methodology and the notations). We can observe in the upper panel of Fig. 10 (A) the output variability. The bacterial levels are quite dispersed around the median (bold black line): large differences are observed between extremal values (dashed red lines), and the bandwidth between the second and third quartiles (grey zone) represents about $20 \%$ of the median value in the middle of the colon.

The lower panel of Figure 10 (A) displays the Sobol index $S_{\theta}(z)$ of the different parameters $\theta$ that are tested, for $z \in(0, L)$, i.e. the contribution of a given parameter to the total variance of the model outputs (see Material and Methods), which is displayed in the upper panel. We can observe that the epithelial motility is the main driver of the spatial structure of the bacterial populations in the proximal colon while the level of fiber input is preponderant in its distal part. 


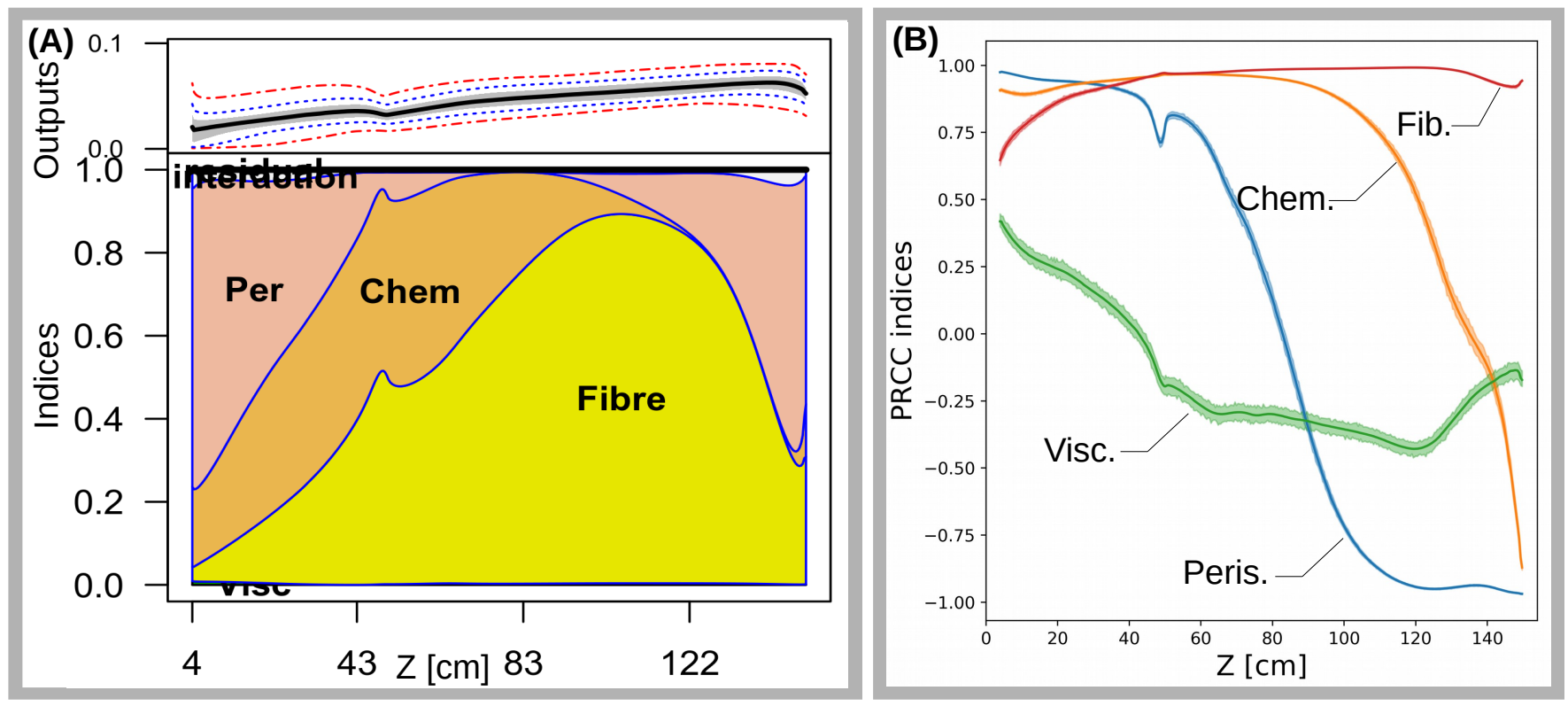

Figure 10: Longitudinal distribution of the first order Sobol and PRCC indices (See Section 3.2.4). We perform a global sensitivity analysis to assess the impact of the parameters driving the peristalsis, the fiber input, the mixture viscosity and the chemotaxis magnitude on the longitudinal distribution of the radially averaged bacterial populations (i.e. on $\left.B(z):=\sum_{i \in I_{\mathrm{B}}} \frac{2}{R^{2}} \int_{0}^{R} r c_{i}(z, r) d r\right)$. We display the first order Sobol (Fig. A) and PRCC (Fig. B) indices. Fig. A: the upper plot displays the dispersion of $B(z)$ when sampling the parameter space by indicating the extremal values (red dashed lines), the first and last deciles (blue dot lines), the second and third quartiles (gray zones) and the median value (black bold line), giving a proxy of the variance of the output. The lower panel displays for each $z$ the first order Sobol index of each parameter, i.e. the part of the total output variance explained by a given parameter. We observe that the epithelial motility is preponderant in the proximal part, while fiber levels is the main driver of the bacterial levels in the transverse and distal compartments. Fig. B We display the PRCC index of the contribution of the different parameters to $B(z)$ together with their $95 \%$ confidence interval. We see that the viscosity is weakly correlated to the output, while the fiber is highly positively correlated to the local bacterial density all along the gut. Chemotaxis and peristalsis are positively correlated at the beginning of the gut but have a negative contribution at the end. Peris.: peristalsis magnitude, Fib.: level of fiber input, Chem.: magnitude of the chemotactic activity, Visc.: mixture viscosity. Interaction: second order Sobol Index.

In the transverse colon, bacterial active swimming and fiber level impacts are equivalent, and the influence of the chemotactic capabilities of the bacteria is noticeable all along the colon, until the very distal part, where diffusion reduces the chemotactic potential gradients. The effect of viscosity variation is very small, with a peak in the very proximal track. This indicates that, despite the necessity of viscosity gradients to obtain physiological bacterial levels (see subsection 3.2 .2 above), the differences between higher and smaller viscosity values are not determinant for the microbial growth: the preponderant mechanism could therefore be related to threshold effects in the sharp distribution of the viscosity values near the mucosa, rather than the effective values of viscosity in the lumen and in the mucus layer. 
The Sobol indices indicate the relative influence of each parameter on the output, but do not provide any insight on the direction of this contribution: the Sobol indices in itself can not show if the parameter positively or negatively impacts the bacterial level. We then supplement the study by computing the PRCC indices, which can be seen as an indicator of the correlation between one parameter and the output. We observe in Figure 10 (B) that the relative magnitude of contribution between parameters exhibited by the Sobol indices is preserved: the viscosity is weakly correlated with the output, while the fiber intake, the peristalsis and the chemotaxis have stronger contributions. The fiber intake has a positive impact all along the gut, while the contribution of the epithelial and bacterial motility is reversed distally. In the proximal gut, the peristalsis have a strong positive impact on the bacterial levels, but this advantage drops in the transverse gut to become strongly negative in the distal gut. This feature could be related to the spatial distribution of the speed fields induced by the epithelial motility: a decrease of the longitudinal speed near the mucosa but an increase in the luminal zone (see Section 3.2.3). Proximally, where the bacteria are mainly located near the mucosa, the speed decrease may provide a fitness advantage, whereas distally, where the microbial radial distribution is more homogeneous, the increased speeds may negatively impact the bacterial growth. The bacterial motility presents a similar pattern: this parameter positively influences the bacterial levels in the proximal colon, but its contribution becomes negative in the more distal part of the gut. A possible mechanism could be the following: in the proximal part, where the convective effects are preponderant, a stiff distribution of the nutritional environment occurs. The motile bacteria get an advantage to reach the mucosal zone where the nutrients accumulate under the effect of water absorption, and where the longitudinal speed is reduced due to the mucus viscosity. Conversely, on the distal part, where the diffusive effect becomes preponderant, the nutrient and the speed distributions are more homogeneous: the active bacteria keep accumulating in the highest nutrient concentration zone, leading to crowd inhibition, while the less active bacteria occupy by diffusion a broader space, favorable enough to grow.

\section{Conclusive discussion}

We introduced a continuous spatio-temporal model of the gut microbiota, that couples a population dynamics model of functional population involved in a trophic chain related to fiber degradation to a fluid mechanic model of the colonic content. A mathematical simplification allowed to reduce the computation time by a factor 70 while keeping the main features. This model was used to investigate the mechanisms driving the spatial distribution of the colonic content and of the microbial populations in the colon. We tested the relative impact of epithelial motility, bacterial active swimming and diet variations through a sensitivity analysis of our model, identifying the later as the preponderant driver of the spatial structure except in the proximal colon, where peristalsis is the main effect, and in the transverse colon, where chemotaxis has an equivalent impact. We furthermore showed that peristalsis and chemotaxis promote the bacterial levels proximally, but decrease the bacterial populations distally. We observed that very low active swimming capabilities are enough to favor the bacterial growth, indicating that this mechanism should not be discarded from spatial studies of 
the gut microbiota. We furthermore exhibited a new mechanism involved in bacterial persistence in the colon, based on radial gradients of viscosity that induce the creation of slow stream zones near the mucosa; these zones can be considered as favorable spatial niches in the vicinity of the mucus layer, which promote the colonization of the luminal part distally.

\subsection{Modeling the gut microbiota in its environment}

Several models of the gut microbiota were proposed in the literature to study the spatial structure of the microbial communities. The present model couples several modeling frameworks that were previously introduced: it adapts the metabolic model presented in [29] to the fluid mechanics model of the mucus and the colonic content defined in [14, while taking into account hydrodynamics balances that were thoroughly studied in 8]. Our spatialization strategy can be compared to the method presented in 28, which was a unidimensional spatialization of [29], but we went deeper into details in the description of the fluid dynamics of the colonic content and we also considered 3D phenomena that can occur in the radial direction of the colon. To our knowledge, the present study introduces the first model that considers the interactions of the gut microbiota with its fluid environment by explicitly coupling a population dynamic model of the microbiota and the key luminal metabolites to a fluid dynamic model of the intestinal flow. This modeling platform is a suitable framework to study the spatial structure of the microbiota and the interactions of the bacterial populations with their environment. As the spatial features are of particular interest during pathogen colonization, this model can be notably adapted to study the spatial host-microbiota-pathogen interactions during infection.

\subsection{Main drivers of the spatial structure of the gut microbiota}

In [8], the balance between bacterial growth and bacterial dilution by the convection was carefully studied, in order to identify a range of colonic content flow allowing bacterial colonization. The author argued that the hydrodynamics alone was not sufficient to reduce the apparent speed in the colon under the dilution threshold, which is a necessary condition for bacterial growth. Several biological mechanisms capable of enforcing the speed reduction or enhancing the retention time were thoroughly discussed and peristalsis was identified as the preponderant mechanism that supplements the hydrodynamics to enable the settlement of bacterial communities.

In the present study, additional fluid mechanic effects were introduced, such as viscosity heterogeneities; they provide low speed zones near the epithelium resulting in the creation of favorable niches in the mucosa vicinity where the local dilution rate is lower than the bacterial growth. We emphasize that such favorable zones were recently observed by imaging a simplified microbiota composed of 15 labelled bacterial strains: high bacterial concentrations were observed in the surroundings of the mucosal wall, but outside the outer mucus layer [43. Viscosity heterogeneities in themselves were sufficient to supplement the basal hydrodynamics in order to make bacterial colonization possible. Additional effects, such as epithelial motility or bacterial active swimming, counter-balance the dilution by the fluid flow and consolidate the bacterial levels in the colon. We thus identified a multifactorial process that includes fluid rheology, peristalsis and active swimming and that leads to the constitution of ecological niches in 
the fluid colonic environment. However, the sensitivity analysis of our model identified the fiber input as the main driver of the microbiota spatial structure, except in the proximal part, where the epithelial motility is determinant for the colonization of the proximal colon. The variations of viscosity gradients weakly impact the bacterial distribution, but an homogeneous viscosity drastically drops down the bacterial populations. This indicates that viscosity heterogeneity is constitutive of a physiological level of bacterial populations, but that the magnitude of these heterogeneities is not preponderant comparatively to the other processes. Threshold effects in the viscosity map near the mucus layer could be the main ingredient of this observation.

Active swimming is often discarded as a possible mechanism enhancing bacterial colonization in the colon, with the arguments that 1) metaproteomic screening of the gut microbiota showed that flagella expression achieved very low levels 42, 2) the maximal known active swimming speeds are in the same order of magnitude than the luminal longitudinal fluid flow, meaning that the bacteria should continuously swim at their maximal capability in order to counter-balance the luminal streams 8,3 ) the flagellin is targeted by the host immune system near the epithelium [10. However, our model showed that very low active swimming (with velocities two orders of magnitude under the longitudinal flow of colonic content, and one to two orders below observed swimmer speeds in biofilms [17]) is enough to noticeably enhance the bacterial levels in the large intestine and structure the microbial communities. It is even the main driving process of the bacterial spatial distribution in the transverse colon, together with fiber level. Our model suggests that the active swimming could be used not to directly face the strong longitudinal streams, since the chemotactic transport in the longitudinal direction is neglected in our model, but to reach the favorable niches near the mucosa, before colonizing the luminal part.

The overall mechanism of the spatial repartition of the colon microbiota could be the following. The bacteria first accumulate near the epithelium with fibers under the effect of water pumping. In the mucosal part, the longitudinal speed is lower, improving the bacterial growth. Viscosity gradients enhanced by the mucus rheology enforce the speed reduction near the mucosa, broadening the favorable niche. Then, by diffusion, the bacterial populations occupy the luminal part more distally. The population front position varies according to nutrient availability: the upper the population substrate in the metabolic pathway, the more proximal the population front. By modulating the underlying transit speed, the peristaltis shifts the longitudinal position of the favorable niches. The bacterial active swimming allows the bacteria to reach these niches more proximally.

\subsection{Model improvements}

Several limitations of our approach can be underlined. First, the description of the bacterial metabolic activity is reduced to a compact version of the fiber degradation pathways leading from carbohydrates to the main end products: lactate, SCFA and gas. This model is built from prior knowledge of fiber degradation and focuses on the metabolism of the main source of substrate in the colon: carbohydrates. But it neglects other secondary processes. Other metabolic activities are activated, such as bile acid degradation or iron sequestration, that could have a significant importance in the spatial structure of the bacterial populations. Some important abiotic parameters were neglected, such as the 
redox balance or the complex acido-basic reactions that modify the $\mathrm{pH}$. If needed for a specific study, the metabolic pathways of our model can be supplemented by additional processes of interest, in a case-by-case basis.

Secondly, several biophysical mechanisms of spatial structuration were ruled out. To face the luminal flow, the bacterial communities can express specific phenotypes. Bacterial aggregation or chain formation may be a collective behavior that was selected for enhancing the friction forces and increasing the retention times in the colon. Several bacteria are also able to bind to materials trapped in the mucus layer, such as DNA strands or lysate residuals: this ability allows them to grow near the carbohydrates incorporated in the mucus which gives them a competitive advantage. These behaviors were not modeled in this study, but can be addressed with classical aggregation models such as Smoluchowski equations, or by adding additional friction terms in the moment conservation equation (9). Furthermore, we did not consider periodic features such as post-prendrial influx or defecation that could impact the overall dynamics: a more detailed modeling of the fluid mechanics should be introduced to integrate these effects.

Finally, if the outputs of the reference model are in the range of observed data, some differences remain: the simulated total microbiota mass at the gut end is half the observation in feces but the simulated total number of bacteria is twice the experimental measures. These discrepancies may reflect the important simplifications that we used in modeling the microbiota. From a metabolic point of view, focusing on fiber metabolism only would lead to underestimate bacterial growth and consequently the overall bacterial levels. Moreover, we do not model the final part of the colon but the colonic content desiccation through water absorption is still active in the sigmoid compartment, which mechanically increases the microbial concentration. This mechanism could account for the discrepancies between our numerical prediction at the gut end and the measured bacterial concentration in feces. Furthermore, the bacterial phases are considered in our model as a homogeneous mixture of liquid and bacteria that forms at a macroscopic scale a viscous fluid: the derivation of bacterial densities expressed in $\mathrm{g} \mathrm{cm}^{-3}$ or $\mathrm{CFU} \mathrm{g}^{-1}$ then relies on assumptions on the average bacterial volume or on the volume saturation by the bacteria in the bacterial phase, which are questionable when modeling bacterial communities with diverse individual shapes and volumes. These modeling issues could be addressed by developing microscale models of the bacterial communities that could be upscaled through mathematical methods such as homogenization in order to better control these approximations. Finally, we note that the definition of the water absorption profile introduced in Section 2.1.5 is determinant to obtain a physiological speed distribution in the colon. The absorption profile that we provide allows for suitable speed distributions, but its piecewise linear distribution introduces small numerical artifacts visible in the different figures at $z \simeq 50 \mathrm{~cm}$ with a small spike, noticeably visible in the radial speed fields, and in the Sobol indices distribution. This artifact, which does not impact the results, could be corrected with a smoother definition of the absorption profile.

\subsection{Suitable data for model assessment}

Linking outputs of spatial models of the gut microbiota to experimental data for assessment or inference purpose is challenging, due to the gap that still remains between the modeled entities and the biological observations. Invasive data can be collected post-mortem: SCFA or bacterial levels [39, 11] were 
measured in some points of the colon, but with very coarse spatial accuracy. The mucus layer thickness was also investigated in rodents [1, revealing the difficulty to clearly identify the loose and the firm mucus layers [19]. Furthermore, the very invasive experimental settings prevented until now the same type of studies in humans 19. These data can be used for a qualitative model calibration, but must be supplemented for parameter inference or quantitative studies.

Omic data can be produced from stool samples, reflecting the state of the gut system at its end: metagenomic data give an insight in the microbial composition of the microbiota and in its functional potential. Metatranscriptomics and metabolomics provide information on the effective expression of microbial functions. In the present study, we could compare levels of specific metabolites, such as SCFA, to measurements in different colonic compartments, or overall bacterial levels. But we have no way to directly link the bacterial levels predicted by our model to metagenomic data, because the modeled bacterial densities are not indexed to any counts of genomic markers. It would be necessary to provide a set of marker genes associated to the different functional populations involved in our model. These marker genes could be a set of $16 \mathrm{~s}$ genes detailing the taxonomic composition of the functional populations that could be compared to $16 s$ counts from stool samples. They could also be a set of genes characterizing the metabolic functions of the functional populations, that could be compared to the corresponding gene counts in shotgun metagenomic data.

Assessing experimentally the spatial structure of the microbiota implies the production of spatial images of the microbial and metabolite distribution. An experimental setup was recently developed [43] in order to track the spatiotemporal evolution of a simplified microbiota of 15 bacterial strains, labelled with a different dye for imaging purpose, and covering the main part of the functions observed in a healthy microbiota. This kind of data set is very promising for assessing spatio-temporal model of the gut microbiota. However, it would need again to modify the structure of the model in order to fit with the specific bacterial populations involved in the experimental study.

Specific experimental settings could be designed to challenge the main results provided by the model. Even if the impact of dietary fiber levels on the microbiota was already proved in humans 12 or in humanized mice [40, the in vivo impact of chemotaxis and epithelial motility seems up to now difficult to investigate. However, cutting edge experimental approaches are currently being developed. A recent study presented transgenic mice with light-sensitive colonic neurons, allowing to control the epithelial motility of the host [15. This mouse model could offer a suitable and nondestructive way to assess the impact of motility on the bacterial levels, for instance by alternatively inducing high or low levels of epithelial motility. The impact of the bacterial motility could be assessed in gnotobiotic mice inoculated with engineered, simplified microbiota. For a particular bacterial species, motile strains could be challenged with non-motile strains. Global (in feces) or local (through fluorescence imaging or local luminal content sampling) competitive indices could be computed to check the fitness advantage provided by chemotaxis and the longitudinal variations of active swimming contribution to bacterial levels. In parallel, as in recent studies using single cell technologies coupled with stable isotopes labelling and fluorescence imaging [5] it could be possible to identify and quantify the metabolic activity of specific bacteria in specific location.

In this context, our model could be a suitable tool to better interpret host- 
commensal interactions near the epithelia, reconsidering the role of the mucus which can provide a favorable zone in its vicinity and not only directly in the looser part of the mucus layer. In the same line, pathogen strategies for gut colonization, that often rely in the invasion of spatial niches, could be interpreted with a broader view: the location of some niches could be related to hydrodynamics features involved in the model.

\section{Acknowledgments}

This publication has been written with the support of the AgreenSkills+ fellowship programme which has received funding from the EU's Seventh Framework Programme under grant agreement Number FP7-609398 (AgreenSkills+ contract) and from the European Research Council (ERC) within the European Union's Horizon 2020 research and innovation programme, under grant agreement No 639638 (MESOPROBIO).

[1] C. Atuma, V. Strugala, A. Allen, and L. Holm. The adherent gastrointestinal mucus gel layer: thickness and physical state in vivo. American Journal of Physiology-Gastrointestinal and Liver Physiology, 280(5):G922-G929, 2001.

[2] E. Barroso, C. Cueva, C. Peláez, M. C. Martínez-Cuesta, and T. Requena. Development of human colonic microbiota in the computer-controlled dynamic simulator of the gastrointestinal tract simgi. LWT-Food Science and Technology, 61(2):283-289, 2015.

[3] G. Bassotti and Edda Battaglia. Physiology of the Colon, pages 43-53. Springer International Publishing, Cham, 2017.

[4] D. J. Batstone, J. Keller, I. Angelidaki, S. Kalyuzhnyi, S. Pavlostathis, A. Rozzi, W. Sanders, H. Siegrist, and V. Vavilin. The iwa anaerobic digestion model no 1 (adm1). Water Science and Technology, 45(10):65-73, 2002 .

[5] D. Berry and A. Loy. Stable-isotope probing of human and animal microbiome function. Trends in microbiology, 2018.

[6] R. Chatelin. Numerical methods for 3D Stokes flow: variable viscosity fluids in a complex moving geometry; application to biological fluids. Phd thesis, Université Paul Sabatier - Toulouse III, November 2013.

[7] F. Clarelli, C. Di Russo, R. Natalini, and M. Ribot. A fluid dynamics model of the growth of phototrophic biofilms. J. Math. Biol., 66(7):1387-1408, 2013.

[8] J. Cremer, M. Arnoldini, and T. Hwa. Effect of water flow and chemical environment on microbiota growth and composition in the human colon. Proceedings of the National Academy of Sciences, page 201619598, 2017.

[9] J. Cremer, I. Segota, C. Yang, M. Arnoldini, J. T. Sauls, Z. Zhang, E. Gutierrez, A. Groisman, and T. Hwa. Effect of flow and peristaltic mixing on bacterial growth in a gut-like channel. Proceedings of the National Academy of Sciences, page 201601306, 2016. 
[10] T. C. Cullender, B. Chassaing, A. Janzon, K. Kumar, C. E. Muller, J. J. Werner, L. T. Angenent, M. E. Bell, A. G. Hay, D. A. Peterson, et al. Innate and adaptive immunity interact to quench microbiome flagellar motility in the gut. Cell host $\&$ microbe, 14(5):571-581, 2013.

[11] J. Cummings, E. Pomare, W. Branch, C. Naylor, and G. Macfarlane. Short chain fatty acids in human large intestine, portal, hepatic and venous blood. Gut, 28(10):1221-1227, 1987.

[12] C. De Filippo, D. Cavalieri, M. Di Paola, M. Ramazzotti, J. B. Poullet, S. Massart, S. Collini, G. Pieraccini, and P. Lionetti. Impact of diet in shaping gut microbiota revealed by a comparative study in children from europe and rural africa. Proceedings of the National Academy of Sciences, 107(33):14691-14696, 2010.

[13] G. P. Donaldson, S. M. Lee, and S. K. Mazmanian. Gut biogeography of the bacterial microbiota. Nature Reviews Microbiology, 14(1):20-32, 2016.

[14] T. El Bouti, T. Goudon, S. Labarthe, B. Laroche, B. Polizzi, A. Rachah, M. Ribot, and R. Tesson. A mixture model for the dynamic of the gut mucus layer. ESAIM: Proceedings and Surveys, 55:111-130, 2016.

[15] T. J. Hibberd, J. Feng, J. Luo, P. Yang, V. K. Samineni, R. W. Gereau, N. Kelley, H. Hu, and N. J. Spencer. Optogenetic induction of colonic motility in mice. Gastroenterology, 2018.

[16] D. Horstmann. Generalizing the keller-segel model: Lyapunov functionals, steady state analysis, and blow-up results for multi-species chemotaxis models in the presence of attraction and repulsion between competitive interacting species. Journal of nonlinear science, 21(2):231-270, 2011.

[17] A. Houry, M. Gohar, J. Deschamps, E. Tischenko, S. Aymerich, A. Gruss, and R. Briandet. Bacterial swimmers that infiltrate and take over the biofilm matrix. Proceedings of the National Academy of Sciences, 109(32):13088 13093, 2012.

[18] . Institute of Medicine. Dietary Reference Intakes for Water, Potassium, Sodium, Chloride, and Sulfate. The National Academies Press, Washington, DC, 2005.

[19] M. E. Johansson, J. M. H. Larsson, and G. C. Hansson. The two mucus layers of colon are organized by the muc2 mucin, whereas the outer layer is a legislator of host-microbial interactions. Proceedings of the national academy of sciences, 108(Supplement 1):4659-4665, 2011.

[20] M. E. Johansson, H. Sjövall, and G. C. Hansson. The gastrointestinal mucus system in health and disease. Nature Reviews Gastroenterology and Hepatology, 10(6):352-361, 2013.

[21] E. F. Keller and L. A. Segel. Initiation of slime mold aggregation viewed as an instability. Journal of Theoretical Biology, 26(3):399-415, 1970.

[22] M. Khashab, P. Pickhardt, D. Kim, and D. Rex. Colorectal anatomy in adults at computed tomography colonography: normal distribution and the effect of age, sex, and body mass index. Endoscopy, 41(08):674-678, 2009. 
[23] M. Lamboni, H. Monod, and D. Makowski. Multivariate sensitivity analysis to measure global contribution of input factors in dynamic models. Reliability Engineering E3 System Safety, 96(4):450-459, 2011.

[24] P. S. Leung. The Gastrointestinal System: Gastrointestinal, Nutritional and Hepatobiliary Physiology. Springer Science \& Business, 2014.

[25] I. Lozoya-Agullo, I. González-Álvarez, M. González-Álvarez, M. MerinoSanjuán, and M. Bermejo. In situ perfusion model in rat colon for drug absorption studies: comparison with small intestine and caco-2 cell model. Journal of pharmaceutical sciences, 104(9):3136-3145, 2015.

[26] M. Minekus, M. Smeets-Peeters, A. Bernalier, S. Marol-Bonnin, R. Havenaar, P. Marteau, M. Alric, G. Fonty, et al. A computer-controlled system to simulate conditions of the large intestine with peristaltic mixing, water absorption and absorption of fermentation products. Applied microbiology and biotechnology, 53(1):108-114, 1999.

[27] K. Molly, M. V. Woestyne, I. D. Smet, and W. Verstraete. Validation of the simulator of the human intestinal microbial ecosystem (shime) reactor using microorganism-associated activities. Microbial Ecology in Health and Disease, 7(4):191-200, 1994.

[28] A. S. Moorthy, S. P. Brooks, M. Kalmokoff, and H. J. Eberl. A spatially continuous model of carbohydrate digestion and transport processes in the colon. PloS one, 10(12):e0145309, 2015.

[29] R. Muñoz-Tamayo, B. Laroche, E. Walter, J. Doré, and M. Leclerc. Mathematical modelling of carbohydrate degradation by human colonic microbiota. J. Theoret. Biol., 266(1):189-201, September 2010.

[30] J. M. Oakes, A. L. Marsden, C. Grandmont, S. C. Shadden, C. Darquenne, and I. Vignon-Clementel. Airflow and Particle Deposition Simulations in Health and Emphysema: From In Vivo to In Silico Animal Experiments. Annals of Biomedical Engineering, 42(4):899-914, April 2014.

[31] B. Polizzi, O. Bernard, and M. Ribot. A time-space model for the growth of microalgae biofilms for biofuel production. Journal of theoretical biology, 432:55-79, 2017.

[32] L. Preziosi and A. Tosin. Multiphase modelling of tumour growth and extracellular matrix interaction: mathematical tools and applications. $J$. Math. Biol., 58(4-5):625-656, 2009.

[33] J. F. Rawls, M. A. Mahowald, A. L. Goodman, C. M. Trent, and J. I. Gordon. In vivo imaging and genetic analysis link bacterial motility and symbiosis in the zebrafish gut. Proceedings of the National Academy of Sciences, 104(18):7622-7627, 2007.

[34] J. L. Round and S. K. Mazmanian. The gut microbiome shapes intestinal immune responses during health and disease. Nature reviews. Immunology, 9(5):313, 2009. 
[35] I. Sekirov, S. L. Russell, L. C. M. Antunes, and B. B. Finlay. Gut microbiota in health and disease. Physiological reviews, 90(3):859-904, 2010.

[36] R. Sender, S. Fuchs, and R. Milo. Revised estimates for the number of human and bacteria cells in the body. PLoS biology, 14(8):e1002533, 2016.

[37] F. Sommer and F. Bäckhed. The gut microbiota-masters of host development and physiology. Nature reviews. Microbiology, 11(4):227, 2013.

[38] P. S. Stewart. Diffusion in biofilms. Journal of bacteriology, 185(5):14851491, 2003.

[39] D. L. Topping and P. M. Clifton. Short-chain fatty acids and human colonic function: roles of resistant starch and nonstarch polysaccharides. Physiological reviews, 81(3):1031-1064, 2001.

[40] P. J. Turnbaugh, V. K. Ridaura, J. J. Faith, F. E. Rey, R. Knight, and J. I. Gordon. The effect of diet on the human gut microbiome: a metagenomic analysis in humanized gnotobiotic mice. Science translational medicine, 1(6):6ra14-6ra14, 2009.

[41] P. Van den Abbeele, S. Roos, V. Eeckhaut, D. A. MacKenzie, M. Derde, W. Verstraete, M. Marzorati, S. Possemiers, B. Vanhoecke, F. Van Immerseel, et al. Incorporating a mucosal environment in a dynamic gut model results in a more representative colonization by lactobacilli. Microbial biotechnology, 5(1):106-115, 2012.

[42] N. C. Verberkmoes, A. L. Russell, M. Shah, A. Godzik, M. Rosenquist, J. Halfvarson, M. G. Lefsrud, J. Apajalahti, C. Tysk, R. L. Hettich, et al. Shotgun metaproteomics of the human distal gut microbiota. The ISME journal, 3(2):179-189, 2009.

[43] J. L. M. Welch, Y. Hasegawa, N. P. McNulty, J. I. Gordon, and G. G. Borisy. Spatial organization of a model 15-member human gut microbiota established in gnotobiotic mice. Proceedings of the National Academy of Sciences, page 201711596, 2017.

[44] D. Winne and W. Verheyen. Diffusion coefficient in native mucus gel of rat small intestine. Journal of Pharmacy and Pharmacology, 42(7):517-519, 1990.

\section{Appendix A. Model parameters and equations}

Appendix A.1. Metabolic activity

We present in Fig. Appendix A.1 the graph of the metabolic capabilities of the microbial populations. The chemical reactions are represented with solid thick arrows and the volume transfers by dashed arrows. The thin dark arrows represent regulation processes such as inhibition or promotion. Complementary information can be found in the Petersen matrices of the processes (Tables Appendix A.1 Appendix A.5) or in the whole set of equations (Section Appendix A.2). In Tables Appendix A.1 and Appendix A.5. $Y_{j, P_{i}}$ denotes the stoichiometric coefficient related to component $j \in I_{\mathrm{C}} \cup I_{\mathrm{S}}$ in process $P_{i}$. 
We recall that $P_{c} \in \mathbb{R}^{8 \times 13}$ and $P_{s} \in \mathbb{R}^{8 \times 13}$ presented in Table Appendix A.1 are the reaction matrices for the mixture components and the solutes that store the yield of each process on the corresponding compounds, based on stoichiometry [29. The kinetic rate vector $K=\left(K_{p}\right)_{p=P 1, \ldots, P 13}$, the components of which are defined by $k_{p} \phi_{p}$ for the different processes $p=P 1, \ldots, P 13$ under consideration, are also recapitulated in Table Appendix A.1. For each process, $k_{p}$ represents a unitary maximal kinetic rate whereas $\varphi_{p}$ models saturation effects. Namely, $\varphi_{p}$ is a Monod-like function for each metabolic process, except for the fiber and mucus hydrolysis, that is modeled with a Comtois law, according to [29] (see Table Appendix A.1.

Appendix A.2. Model equations and parameters

All the unknowns are summarized in Table Appendix A.2, we can now write the full set of equations for the model :

Mass balance equations for the mixture components :

$$
\begin{aligned}
& \partial_{t} f_{m}-\operatorname{div}\left(\sigma \nabla f_{m}\right)+\operatorname{div}\left(f_{m} u\right)=-k_{P 1} \frac{f_{m} \cdot f_{\mathrm{B}_{m o n}}}{K_{x, P 1} f_{\mathrm{B}_{m o n}}+f_{m}} \\
& \partial_{t} f_{p o l}-\operatorname{div}\left(\sigma \nabla f_{p o l}\right)+\operatorname{div}\left(f_{p o l} u\right)=-k_{P 2} \frac{f_{p o l} \cdot f_{\mathrm{B}_{m o n}}}{K_{x, P 2} f_{\mathrm{B}_{m o n}}+f_{p o l}} \\
& \partial_{t} f_{\mathrm{B}_{m o n}}-\operatorname{div}\left(\sigma \nabla f_{\mathrm{B}_{m o n}}\right)+\operatorname{div}\left(f_{\mathrm{B}_{m o n}} u_{\mathrm{B}_{m o n}}\right)=k_{P 3} Y_{\mathrm{B}_{m o n}, P 3} f_{l} \frac{c_{m o n} \cdot f_{\mathrm{B}_{m o n}}}{K_{s, P 3}+c_{m o n}}-k_{P 7} f_{\mathrm{B}_{m o n}} \\
& \partial_{t} f_{\mathrm{B}_{l a}}-\operatorname{div}\left(\sigma \nabla f_{\mathrm{B}_{l a}}\right)+\operatorname{div}\left(f_{\mathrm{B}_{l a}} u_{\mathrm{B}_{l a}}\right)=k_{P 4} Y_{\mathrm{B}_{l a}, P 4} f_{l} \frac{c_{l a} \cdot f_{\mathrm{B}_{l a}}}{K_{s, P 4}+c_{l a}}-k_{P 8} f_{\mathrm{B}_{l a}} \\
& \partial_{t} f_{\mathrm{B}_{H_{2} a}}-\operatorname{div}\left(\sigma \nabla f_{\mathrm{B}_{H_{2} a}}\right)+\operatorname{div}\left(f_{\mathrm{B}_{H_{2} a}} u_{\mathrm{B}_{H_{2} a}}\right)=k_{P 5} Y_{\mathrm{B}_{H_{2} a}, P 5} f_{l} \frac{c_{H_{2}} \cdot f_{\mathrm{B}_{H_{2} a}}}{K_{s, P 5}+c_{H_{2}}}-k_{P 9} f_{\mathrm{B}_{H_{2} a}} \\
& \partial_{t} f_{\mathrm{B}_{H_{2}} m}-\operatorname{div}\left(\sigma \nabla f_{\mathrm{B}_{H_{2} m}}\right)+\operatorname{div}\left(f_{\mathrm{B}_{H_{2} m}} u_{\mathrm{B}_{H_{2} m}}\right)=k_{P 6} Y_{\mathrm{B}_{H_{2}}, P 6} f_{l} \frac{c_{H_{2}} \cdot f_{\mathrm{B}_{H_{2} m}}}{K_{s, P 6}+c_{H_{2}}} I_{p H}-k_{P 10} f_{\mathrm{B}_{H_{2} m}} \\
& \partial_{t} f_{r}-\operatorname{div}\left(\sigma \nabla f_{r}\right)+\operatorname{div}\left(f_{r} u\right)=0 \\
& \partial_{t} f_{l}-\operatorname{div}\left(\sigma \nabla f_{l}\right)+\operatorname{div}\left(f_{l} u\right)=k_{P 1} \frac{f_{m} \cdot f_{\mathrm{B}_{m o n}}}{K_{x, P 1} f_{\mathrm{B}_{m o n}}+f_{m}}+k_{P 2} \frac{f_{p o l} \cdot f_{\mathrm{B}_{m o n}}}{K_{x, P 2} f_{\mathrm{B}_{m o n}}+f_{p o l}} \\
& -k_{P 3} Y_{\mathrm{B}_{m o n}, P 3} f_{l} \frac{c_{m o n} \cdot f_{\mathrm{B}_{m o n}}}{K_{s, P 3}+c_{m o n}}-k_{P 4} Y_{\mathrm{B}_{l a}, P 4} f_{l} \frac{c_{l a} \cdot f_{\mathrm{B}_{l a}}}{K_{s, P 4}+c_{l a}}-k_{P 5} Y_{\mathrm{B}_{H_{2} a}, P 5} f_{l} \frac{c_{H_{2}} \cdot f_{\mathrm{B}_{H_{2 a}}}}{K_{s, P 5}+c_{H_{2}}} \\
& -k_{P 6} Y_{\mathrm{B}_{H_{2}}, P 6} f_{l} \frac{c_{H_{2}} \cdot f_{\mathrm{B}_{H_{2 m}}}}{K_{s, P 6}+c_{H_{2}}} I_{p H}+k_{P 7} f_{\mathrm{B}_{m o n}}+k_{P 8} f_{\mathrm{B}_{l a}}+k_{P 9} f_{\mathrm{B}_{H_{2} a}}+k_{P 10} f_{\mathrm{B}_{H_{2} m}} \\
& \text { where } I_{p H}(z):=\exp \left(-3\left(\frac{p H(z)-I_{p H, \text { high }}}{I_{p H, \text { high }}-I_{p H, \text { low }}}\right)^{2}\right) \mathbf{1}_{p H(z)<I_{p H, \text { high }}}+\mathbf{1}_{p H(z) \geq I_{p H, \text { high }}} \\
& \text { and } p H(z)=I_{p H, \text { min }}+\left(I_{p H, \text { max }}-I_{p H, \min }\right) z / L
\end{aligned}
$$

Volume constraint :

$$
\sum_{i \in I_{\mathrm{C}}} f_{i}(t, x)=1
$$



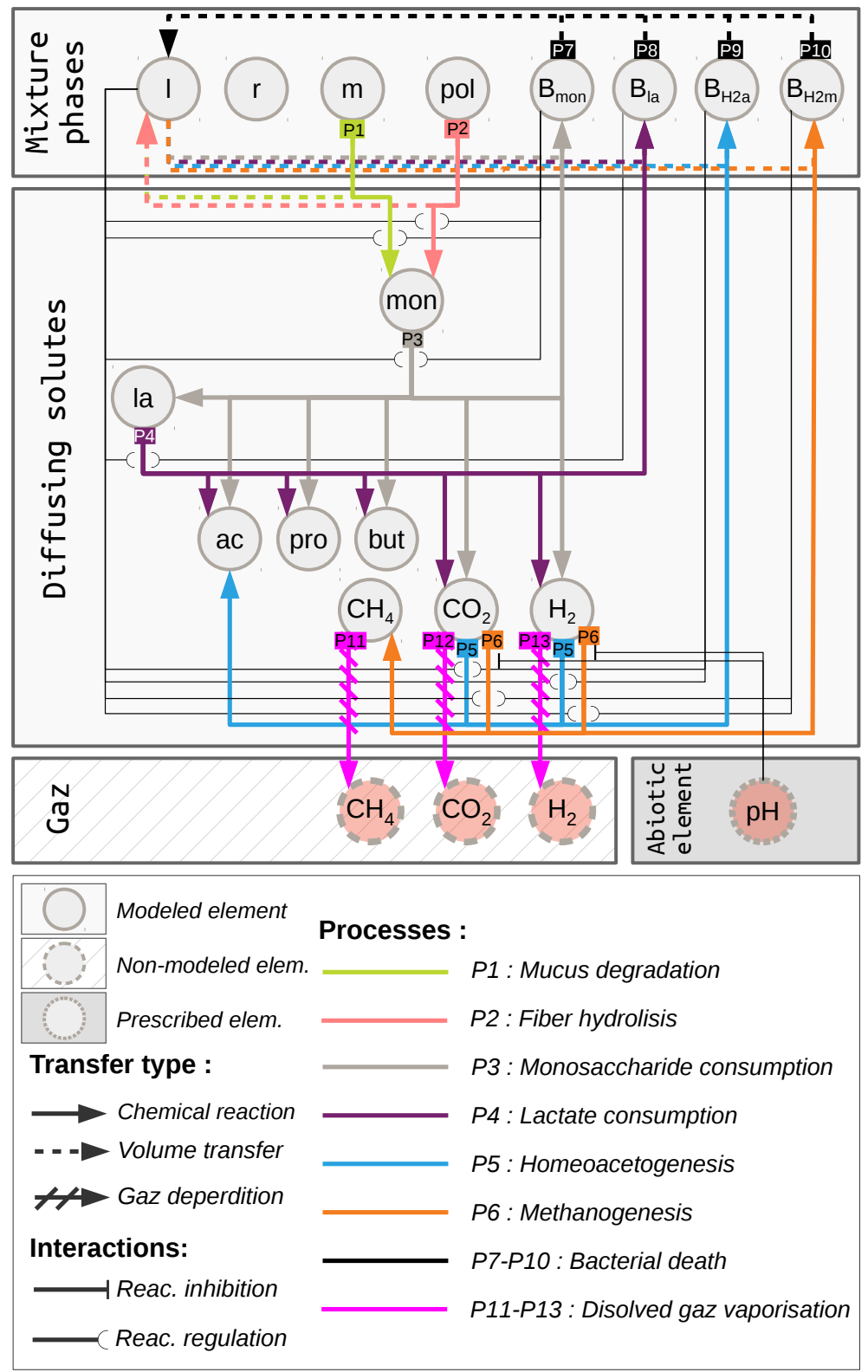

Figure Appendix A.1: Graph of the reactions and volume transfers considered in the model. We represent the functional interactions between the mixture components of $I_{\mathrm{C}}$ (liquid $l$, digestive residuals $r$, mucus $m$, polysaccharides pol, and the bacterial populations $\mathrm{B}_{m o n}, \mathrm{~B}_{l a}, \mathrm{~B}_{H 2 a}$ and $\mathrm{B}_{H 2 m}$ ) and the diffusing solutes of $I_{\mathrm{S}}$ (monosaccharides mon, lactate $l a$ acetate ac, propionate pro, butyrate but, methane $\mathrm{CH}_{4}$, carbone dioxyde $\mathrm{CO}_{2}$ and hydrogen $\mathrm{H}_{2}$ ). We represent in the graph gazeous components, but they are not explicitly modeled: they represent sink sources for the corresponding diffusing solutes. A fixed $\mathrm{pH}$ gradient is prescribed on the domain: it interacts with the process P6. 


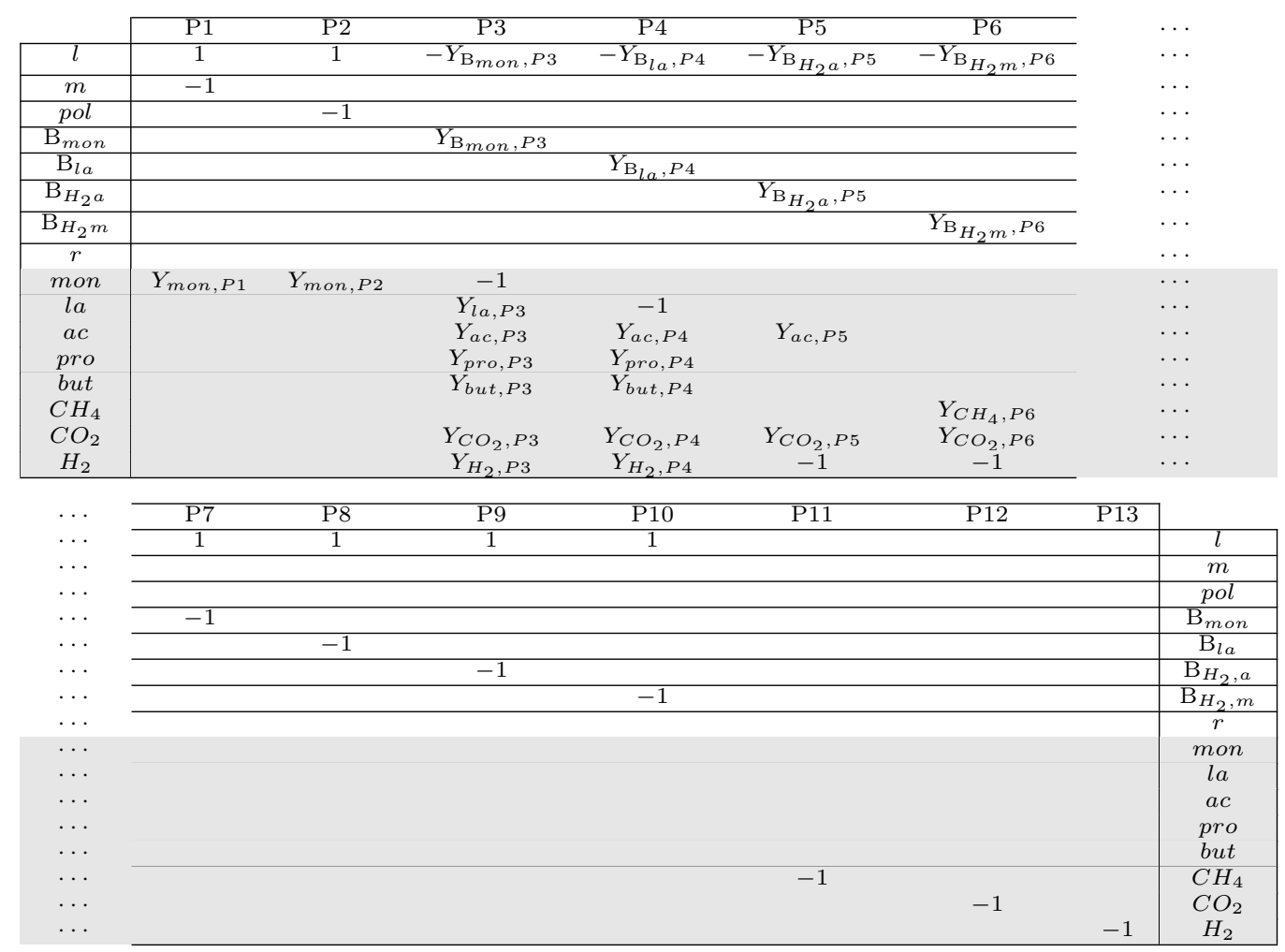

(a) Reaction matrix $P_{c}$ (white background) and $P_{s}$ (grey background).

\begin{tabular}{|c|c|c|c|c|c|}
\hline & P1 & $\mathrm{P} 2$ & P3 & $\mathrm{P} 4$ & P5 \\
\hline$\phi_{p}$ & $\frac{f_{m} \cdot f_{\mathrm{B} m o n}}{K_{x, P 1} f_{\mathrm{B}_{m o n}}+f_{m}}$ & $\frac{f_{p o l} \cdot f_{\mathrm{B}_{m o n}}}{K_{x, P 2} f_{\mathrm{B}_{m o n}}+f_{p o l}}$ & $f_{l} \frac{c_{m o n} \cdot f_{\mathrm{B} m o n}}{K_{s, P 3}+c_{m o n}}$ & $f_{l} \frac{c_{l a} \cdot f_{\mathrm{B}_{l a}}}{K_{s, P 4}+c_{l a}}$ & $f_{l}{ }^{{ }^{c} H_{2} \cdot f^{-} \mathrm{B}_{H_{2 a}}} \frac{K_{s, P 5}+{ }^{c} H_{2}}{K_{2}}$ \\
\hline$k_{p}$ & $\frac{k_{P 1}}{P 6}$ & $\frac{k_{P 2}}{P_{7}}$ & $\frac{k_{P 3}}{P 8}$ & $\frac{k_{P 4}}{P 9}$ & $\frac{k_{P 5}}{\mathrm{P} 10}$ \\
\hline$\phi_{p}$ & 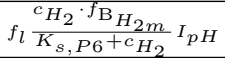 & $f_{\mathrm{B}_{m o n}}$ & $f_{\mathrm{B}_{l a}}$ & ${ }^{f_{\mathrm{B}}}{ }_{2 a}$ & $f_{\mathrm{B}_{H_{2 m}}}$ \\
\hline$k$ & $k_{P 6}$ & $k_{P 7}$ & $k_{P 8}$ & $k_{P 9}$ & $k_{P 10}$ \\
\hline & \multicolumn{2}{|c|}{ P11 } & \multicolumn{2}{|c|}{$\overline{\mathrm{P} 12}$} & P13 \\
\hline$\phi_{p}$ & \multirow{2}{*}{\multicolumn{2}{|c|}{$\frac{{ }^{c} C H_{4}-K_{h, P 11} R T\left[C H_{4, g}\right]_{\infty}}{k_{P 11}}$}} & \multirow{2}{*}{\multicolumn{2}{|c|}{$\frac{{ }^{c} \mathrm{CO}_{2}-K_{h, P 12} R T\left[\mathrm{CO}_{2, g}\right]_{\infty}}{k_{P 12}}$}} & ${ }^{c} \mathrm{H}_{2}-K_{h, P 13} R T\left[H_{2, g}\right]_{\infty}$ \\
\hline$k$ & & & & & $k_{P 13}$ \\
\hline
\end{tabular}

(b) Vector of the kinetic rates. For $p=P 1, \ldots, P 13$, we have $K=\left(K_{p}\right)_{p=P 1, \ldots, P 13}:=k_{p} \rho_{p}$.

Table Appendix A.1: Petersen matrices and kinetic rate vectors. 


\begin{tabular}{|c|c|c|c|}
\hline \multicolumn{4}{|c|}{ Unknowns - volume fractions of mixture components } \\
\hline Unknown & Unit & Description & Equation \\
\hline$f_{m}$ & {$[-]$} & Volume fraction of mucus & (1) \\
\hline$f_{\text {pol }}$ & {$[-]$} & Volume fraction of polysaccharides & 11 \\
\hline$f_{l}$ & {$[-]$} & Volume fraction of liquid & 1 \\
\hline$f_{r}$ & {$[-]$} & Volume fraction of digestible residuals & 1 \\
\hline$f_{\mathrm{B}_{m o n}}$ & {$[-]$} & Volume fraction of bacteria metabolizing monosaccharides & (1) \\
\hline$f_{\mathrm{B}_{l a}}$ & {$[-]$} & Volume fraction of bacteria metabolizing lactate & 1 \\
\hline$f_{\mathrm{B}_{H_{2} a}}$ & {$[-]$} & Volume fraction of bacteria producing acetate from $\mathrm{H}_{2}$ & (1) \\
\hline$f_{\mathrm{B}_{H_{2} m}}$ & {$[-]$} & Volume fraction of bacteria producing methane from $H_{2}$ & 11 \\
\hline \multicolumn{4}{|c|}{ Unknowns - diffusing compounds concentrations } \\
\hline Unknown & Unit & Description & Equation \\
\hline$c_{m o n}$ & mol.cm ${ }^{-3}$ & Concentration of monosaccharides & 5 \\
\hline$c_{l a}$ & mol.cm ${ }^{-3}$ & Concentration of lactate & 5 \\
\hline$c_{\mathrm{H}_{2}}$ & mol.cm ${ }^{-3}$ & Concentration of dissolveddi-hydrogen & 5 \\
\hline$c_{a c}$ & mol.cm ${ }^{-3}$ & Concentration of acetate & 5 \\
\hline$c_{\text {pro }}$ & mol.cm ${ }^{-3}$ & Concentration of propionate & 5 \\
\hline$c_{b u}$ & mol.cm ${ }^{-3}$ & Concentration of butyrate & $(5)$ \\
\hline$c_{C H_{4}}$ & mol.cm ${ }^{-3}$ & Concentration of methane & $(5)$ \\
\hline$c_{\mathrm{CO}_{2}}$ & mol.cm ${ }^{-3}$ & Concentration of dissolved carbone dioxyde & $(5)$ \\
\hline \multicolumn{4}{|c|}{ Unknowns - velocities } \\
\hline$u$ & $\mathrm{~cm} \cdot \mathrm{d}^{-1}$ & Average mixture velocity & (6) \\
\hline$\tilde{u}$ & $\mathrm{~cm} \cdot \mathrm{d}^{-1}$ & Average velocity for the dissolved constituents & 4 \\
\hline$u_{m}$ & $\mathrm{~cm} \cdot \mathrm{d}^{-1}$ & Velocity field for mucus & 6 \\
\hline$u_{\text {pol }}$ & $\mathrm{cm} \cdot \mathrm{d}^{-1}$ & Velocity field for polysaccharides & 6 \\
\hline$u_{l}$ & $\mathrm{~cm} \cdot \mathrm{d}^{-1}$ & Velocity field for liquid & (6) \\
\hline$u_{r}$ & $\mathrm{~cm} \cdot \mathrm{d}^{-1}$ & Velocity field for digestible residuals & 6 \\
\hline$u_{\mathrm{B}_{m o n}}$ & $\mathrm{~cm} \cdot \mathrm{d}^{-1}$ & Velocity field for bacteria $\mathrm{B}_{m o n}$ (with chemotaxis) & $(6)$ \\
\hline$u_{\mathrm{B}_{l a}}$ & $\mathrm{~cm} \cdot \mathrm{d}^{-1}$ & Velocity field for bacteria $\mathrm{B}_{l a}$ (with chemotaxis) & 6 \\
\hline$u_{\mathrm{B}_{H_{2}} a}$ & $\mathrm{~cm} \cdot \mathrm{d}^{-1}$ & Velocity field for bacteria $\mathrm{B}_{H_{2} a}$ (with chemotaxis) & 6 \\
\hline$u_{\mathrm{B}_{H_{2} m}}$ & $\mathrm{~cm} \cdot \mathrm{d}^{-1}$ & Velocity field for bacteria $\mathrm{B}_{H_{2} m}$ (with chemotaxis) & 6 \\
\hline$\vartheta_{\mathrm{B}_{m o n}, \text { chem }}$ & $\mathrm{cm} \cdot \mathrm{d}^{-1}$ & Chemotactic velocity field for bacteria $\mathrm{B}_{m o n}$ & 10 \\
\hline$\vartheta_{\mathrm{B}_{l a}, \text { chem }}$ & $\mathrm{cm} \cdot \mathrm{d}^{-1}$ & Chemotactic velocity field for bacteria $\mathrm{B}_{l a}$ & 10 \\
\hline$\vartheta_{\mathrm{B}_{H_{2} a}, \text { chem }}$ & $\mathrm{cm} \cdot \mathrm{d}^{-1}$ & Chemotactic velocity field for bacteria $\mathrm{B}_{H_{2}} a$ & 10 \\
\hline$\vartheta_{\mathrm{B}_{H_{2} m}, \text { chem }}$ & $\mathrm{cm} \cdot \mathrm{d}^{-1}$ & Chemotactic velocity field for bacteria $\mathrm{B}_{H_{2} m}$ & 10 \\
\hline \multicolumn{4}{|c|}{ Unknowns - chemotactic potential } \\
\hline$\Phi_{m}$ & $\mathrm{~cm}^{2}$ & Chemotactic potential produced by mucus & 11 \\
\hline$\Phi_{\text {pol }}$ & $\mathrm{cm}^{2}$ & Chemotactic potential produced by polysaccharides & 11 \\
\hline$\Phi_{m o n}$ & mol.cm ${ }^{-1}$ & Chemotactic potential produced by monosaccharides & 12 \\
\hline$\Phi_{l a}$ & mol.cm ${ }^{-1}$ & Chemotactic potential produced by lactate & 12 \\
\hline$\Phi_{\mathrm{H}_{2}}$ & mol.cm ${ }^{-1}$ & Chemotactic potential produced by di-hydrogen & 12 \\
\hline \multicolumn{4}{|c|}{ Unknowns - pressure and viscosity } \\
\hline$p$ & g. $\mathrm{cm}^{-1} \cdot \mathrm{d}^{-2}$ & Pressure of the mixture & 9 \\
\hline$\mu$ & g.cm ${ }^{-1} \cdot \mathrm{d}^{-1}$ & Mixture viscosity & 15. \\
\hline$\mu_{m}$ & g.cm ${ }^{-1} \cdot \mathrm{d}^{-1}$ & Description of the mucus rheology & 16 \\
\hline$\mu_{l}$ & g.cm ${ }^{-1} \cdot d^{-1}$ & Description of the liquid rheology & 17) \\
\hline \multicolumn{4}{|c|}{ Unknowns - source functions } \\
\hline$\overline{F_{i}}$ & $d^{-1}$ & Source function for the component $i \in I_{\mathrm{C}}$ & (1) $-(13)$ \\
\hline$G_{j}$ & $d^{-1}$ & Source function for the component $j \in I_{\mathrm{S}}$ & -13 \\
\hline$K$ & $d^{-1}$ & Kinetic rate vector & $\sqrt{13)}$ \\
\hline$\phi_{p}$ & {$[-]$} & Function modelling saturation effects for process $p$ & - \\
\hline \multicolumn{4}{|c|}{ Unknowns - boundary conditions } \\
\hline$\gamma_{f_{i}}$ & $\mathrm{~cm} \cdot \mathrm{d}^{-1}$ & Boundary flux for component $i \in I_{\mathrm{C}}$ & $\frac{14}{14}$ \\
\hline$\gamma_{c_{i}}$ & mol.cm $\cdot \mathrm{d}$ & Boundary flux for component $\jmath \in I_{\mathrm{S}}$ & \\
\hline
\end{tabular}

Table Appendix A.2: Table of the unknowns of the model 
Mass balance equations for the dissolved compounds :

$$
\begin{aligned}
& \partial_{t} c_{m o n}-\operatorname{div}\left(\sigma_{m o n} \nabla c_{m o n}\right)+\operatorname{div}\left(c_{m o n} \tilde{u}\right)=k_{P 1} Y_{m o n}, P 1 \frac{f_{m} \cdot f_{\mathrm{B}_{m o n}}}{K_{x, P 1} f_{\mathrm{B}_{m o n}}+f_{m}} \\
& +k_{P 2} Y_{m o n, P 2} \frac{f_{p o l} \cdot f_{\mathrm{B}_{m o n}}}{K_{x, P 2} f_{\mathrm{B}_{m o n}}+f_{p o l}}-k_{P 3} f_{l} \frac{c_{m o n} \cdot f_{\mathrm{B}_{m o n}}}{K_{s, P 3}+c_{m o n}} \\
& \partial_{t} c_{l a}-\operatorname{div}\left(\sigma_{l a} \nabla c_{l a}\right)+\operatorname{div}\left(c_{l a} \tilde{u}\right)=k_{P 3} Y_{l a, P 3} f_{l} \frac{c_{m o n} \cdot f_{\mathrm{B}_{m o n}}}{K_{s, P 3}+c_{m o n}}-k_{P 4} f_{l} \frac{c_{l a} \cdot f_{\mathrm{B}_{l a}}}{K_{s, P 4}+c_{l a}} \\
& \partial_{t} c_{a c}-\operatorname{div}\left(\sigma_{a c} \nabla c_{a c}\right)+\operatorname{div}\left(c_{a c} \tilde{u}\right)=k_{P 3} Y_{a c, P 3} f_{l} \frac{c_{m o n} \cdot f_{\mathrm{B}_{m o n}}}{K_{s, P 3}+c_{m o n}}+k_{P 4} Y_{a c, P 4} f_{l} \frac{c_{l a} \cdot f_{\mathrm{B}_{l a}}}{K_{s, P 4}+c_{l a}} \\
& +k_{P 5} Y_{a c, P 5} f_{l} \frac{c_{H_{2}} \cdot f_{\mathrm{B}_{H_{2 a}}}}{K_{s, P 5}+c_{H_{2}}} \\
& \partial_{t} c_{\text {pro }}-\operatorname{div}\left(\sigma_{\text {pro }} \nabla c_{\text {pro }}\right)+\operatorname{div}\left(c_{\text {pro }} \tilde{u}\right)=k_{P 3} Y_{\text {pro }, P 3} f_{l} \frac{c_{m o n} \cdot f_{\mathrm{B}_{\text {mon }}}}{K_{s, P 3}+c_{m o n}}+k_{P 4} Y_{\text {pro }, P 4} f_{l} \frac{c_{l a} \cdot f_{\mathrm{B}_{l a}}}{K_{s, P 4}+c_{l a}} \\
& \partial_{t} c_{b u t}-\operatorname{div}\left(\sigma_{b u t} \nabla c_{b u t}\right)+\operatorname{div}\left(c_{b u t} \tilde{u}\right)=k_{P 3} Y_{b u t, P 3} f_{l} \frac{c_{m o n} \cdot f_{\mathrm{B}_{m o n}}}{K_{s, P 3}+c_{m o n}}+k_{P 4} Y_{b u t, P 4} f_{l} \frac{c_{l a} \cdot f_{\mathrm{B}_{l a}}}{K_{s, P 4}+c_{l a}} \\
& \partial_{t} c_{C H_{4}}-\operatorname{div}\left(\sigma_{C H_{4}} \nabla c_{C H_{4}}\right)+\operatorname{div}\left(c_{C H_{4}} \tilde{u}\right)=k_{P 6} Y_{C H_{4}, P 6} f_{l} \frac{c_{H_{2}} \cdot f_{\mathrm{B}_{H_{2 m}}}}{K_{s, P 6}+c_{H_{2}}} I_{p H} \\
& -k_{P 11}\left(c_{C H_{4}}-K_{h, P 11} R T\left[C_{4, g}\right]_{\infty}\right) \\
& \partial_{t} c_{C_{2}}-\operatorname{div}\left(\sigma_{C_{2}} \nabla c_{C O_{2}}\right)+\operatorname{div}\left(c_{C O_{2}} \tilde{u}\right)=k_{P 3} Y_{C O_{2}, P 3} f_{l} \frac{c_{m o n} \cdot f_{\mathrm{B}_{m o n}}}{K_{s, P 3}+c_{m o n}} \\
& +k_{P 4} Y_{C O_{2}, P 4} f_{l} \frac{c_{l a} \cdot f_{\mathrm{B}_{l a}}}{K_{s, P 4}+c_{l a}}+k_{P 5} Y_{C O_{2}, P 5} f_{l} \frac{c_{H_{2}} \cdot f_{\mathrm{B}_{H_{2 a}}}}{K_{s, P 5}+c_{H_{2}}}+k_{P 6} Y_{C O_{2}, P 6} f_{l} \frac{c_{H_{2}} \cdot f_{\mathrm{B}_{H_{2 m}}}}{K_{s, P 6}+c_{H_{2}}} I_{p H} \\
& -k_{P 12}\left(c_{C O_{2}}-K_{h, P 12} R T\left[C_{2, g}\right]_{\infty}\right) \\
& \partial_{t} c_{H_{2}}-\operatorname{div}\left(\sigma_{H_{2}} \nabla c_{H_{2}}\right)+\operatorname{div}\left(c_{H_{2}} \tilde{u}\right)=k_{P 3} Y_{H_{2}, P 3} f_{l} \frac{c_{m o n} \cdot f_{\mathrm{B}_{m o n}}}{K_{s, P 3}+c_{m o n}}+k_{P 4} Y_{H_{2}, P 4} f_{l} \frac{c_{l a} \cdot f_{\mathrm{B}_{l a}}}{K_{s, P 4}+c_{l a}} \\
& -k_{P 5} f_{l} \frac{c_{H_{2}} \cdot f_{\mathrm{B}_{H_{2 a}}}}{K_{s, P 5}+c_{H_{2}}}-k_{P 6} f_{l} \frac{c_{H_{2}} \cdot f_{\mathrm{B}_{H_{2 m}}}}{K_{s, P 6}+c_{H_{2}}} I_{p H}-k_{P 13}\left(c_{H_{2}}-K_{h, P 13} R T\left[H_{2, g}\right]_{\infty}\right)
\end{aligned}
$$

Stokes equation for the velocity field of the carrying fluid :

$$
\begin{aligned}
& \nabla p-\frac{1}{2} \operatorname{div}\left(\mu\left(f_{m}, f_{l}\right)\left(\nabla u+\nabla u^{\top}\right)\right)=0, \\
& \quad \text { with } \mu\left(f_{m}, f_{l}\right)=\max _{k=m, l}\left(\mu_{k, \text { min }}+\left(\mu_{k, \text { max }}-\mu_{k, \text { min }}\right) \frac{f_{k}^{\alpha_{k}}}{f_{k, t h r}^{\alpha_{k}}+f_{k}^{\alpha_{k}}}\right)
\end{aligned}
$$

\section{Definition of the velocities :}

$u_{i}=u+\vartheta_{i, c h e m}$, where $\vartheta_{i, c h e m}=\sum_{j} \lambda_{i j} \nabla \Phi_{j}$, if $i \in I_{\mathrm{B}}=\left\{\mathrm{B}_{m o n}, \mathrm{~B}_{l a}, \mathrm{~B}_{H_{2} a}, \mathrm{~B}_{H_{2} m}\right\}$

$\tilde{u}=u+\sum_{i \in I_{\mathrm{B}}} f_{i} \vartheta_{i, c h e m}$ 
Poisson equations for the chemotatic potential :

$-\Delta \Phi_{j}=f_{j}-\frac{1}{|\omega|} \int_{\omega} f_{j}(x, z) \mathrm{d} x$ in $\Omega, \quad \nabla \Phi_{j} \cdot \eta=0$ on $\partial \Omega, \quad$ when $j \in\{m, p o l\}$

$-\Delta \Phi_{j}=c_{j}-\frac{1}{|\omega|} \int_{\omega} c_{j}(x, z) \mathrm{d} x$ in $\Omega, \quad \nabla \Phi_{j} \cdot \eta=0$ on $\partial \Omega, \quad$ when $j \in\left\{m o n, l a, H_{2}\right\}$

\section{Boundary conditions :}

On $\Gamma_{i n}$ :

$$
\begin{aligned}
& u \cdot \eta=U_{\text {in }}=V_{\text {in }} /|\omega| \\
& \left(-\sigma \nabla f_{i}+f_{i} u\right) \cdot \eta=U_{i n} f_{i, i n} \text {, when } i \in I_{\mathrm{C}} \\
& \left(-\sigma_{j} \nabla c_{j}+c_{j} u\right) \cdot \eta= \begin{cases}U_{i n} c_{j, i n}, & \text { when } j=\text { mon } \\
0, & \text { when } j \in I_{\mathrm{S}} \backslash\{m o n\}\end{cases}
\end{aligned}
$$

On $\Gamma_{m}$ :

$$
\begin{aligned}
& \left(-\sigma \nabla f_{m}+f_{m} u\right) \cdot \eta=g_{m}\left(f_{m}-f_{m}^{*}\right) \\
& \left(-\sigma \nabla f_{l}+f_{l} u\right) \cdot \eta=\left\{\begin{array}{l}
g_{l, \text { max }} f_{l} \text { for } z \in\left[0, L_{\text {prox }}\right], \\
\left(g_{l, \text { max }}-\frac{z-L_{\text {prox }}}{L_{\text {trans }}}\left(g_{l, \text { max }}-g_{l, \text { min }}\right)\right) f_{l} \text { for } z \in\left[L_{\text {prox }}, L_{\text {prox }}+L_{\text {trans }}\right], \\
g_{l, \text { min }} f_{l} \text { for } z \in\left[L_{\text {prox }}+L_{\text {trans }}, L\right]
\end{array}\right.
\end{aligned}
$$

$\left(-\sigma \nabla f_{i}+f_{i} u\right) \cdot \eta=0$, when $i \in I_{\mathrm{C}} \backslash\{l, m\}=\left\{p o l, r, \mathrm{~B}_{m o n}, \mathrm{~B}_{l a}, \mathrm{~B}_{H_{2} a}, \mathrm{~B}_{H_{2} m}\right\}$

$\left(-\sigma_{j} \nabla c_{j}+c_{j} u\right) \cdot \eta=g_{j, \max }-\left(g_{j, \max }-g_{j, \min }\right) \frac{z}{L}$ for $j \in\{l a, a c, p r o, b u\}$,

$\left(-\sigma_{j} \nabla c_{j}+c_{j} u\right) \cdot \eta=0$ when $j \in\left\{\right.$ mon, $\left.\mathrm{H}_{2}, \mathrm{CH}_{4}, \mathrm{CO}_{2}\right\}$,

$u_{i} \cdot \eta=\sum_{i \in I_{\mathrm{C}}} \gamma_{f_{i}}+U_{p e r, r}, \quad u_{i} \cdot \eta_{z}=U_{p e r, z}$.

On $\Gamma_{\text {out }}$ :

$$
\begin{aligned}
& \left(-\sigma \nabla f_{i}+f_{i} u\right) \cdot \eta=f_{i} u_{i} \cdot \eta, \text { when } i \in I_{\mathrm{C}} \\
& \left(-\sigma_{j} \nabla c_{j}+c_{j} u\right) \cdot \eta=c_{j} u \cdot \eta, \text { when } j \in I_{\mathrm{S}}
\end{aligned}
$$

We recall that those previous equations are not directly solved numerically. Instead, we solve their asymptotic approximations (21)-22 with the speed approximations (25)- 26 ) and the approximations of the chemotactic potentials 23)-24.

\section{Appendix B. Supplementary materials}

In this annex, we present 6 supplementary figures :

- Figure Appendix B.1 displays the spatial distribution in the transverse gut of the four bacterial populations $\mathrm{B}_{m o n}, \mathrm{~B}_{l a}, \mathrm{~B}_{H_{2} a}$ and $\mathrm{B}_{H_{2} m}$ and their nutriments : monosaccharides, lactate and di-hydrogen, where we can observe that the bacterial populations are higher in the vicinity of the mucus layer and lower in the lumen (see Sections 3.1.2 and 3.1.3), 


\begin{tabular}{|c|c|c|c|}
\hline \multicolumn{4}{|c|}{ Model unknowns of the asymptotic model: mixture components } \\
\hline Unknown & Unit & Description & Equation \\
\hline$f_{m}$ & {$[-]$} & Mucus volume fraction approximation & 21 \\
\hline$f_{\text {pol }}$ & {$[-]$} & Polysaccharide volume fraction approximation & 21 \\
\hline$f_{\mathrm{B}_{m o n}}$ & {$[-]$} & Approx. of the volume fraction of $\mathrm{B}_{\text {mon }}$ & 21 \\
\hline$f_{\mathrm{B}_{l a}}$ & {$[-]$} & Approx. of the volume fraction of $\mathrm{B}_{l a}$ & 21 \\
\hline$f_{\mathrm{B}_{H_{2}} a}$ & {$[-]$} & Approx. of the volume fraction of $\mathrm{B}_{\mathrm{H}_{2} a}$ & 21 \\
\hline$f_{\mathrm{B}_{H_{2} m}}$ & {$[-]$} & Approx. of the volume fraction of $\mathrm{B}_{H_{2} m}$ & 21 \\
\hline$f_{l}$ & {$[-]$} & Liquid chyme volume fraction approximation & 21 \\
\hline$f_{r}$ & {$[-]$} & Undigestible residual volume fraction approximation & 21 \\
\hline \multicolumn{4}{|c|}{ Model unknowns of the asymptotic model: diffusing compounds } \\
\hline Unknown & Unit & Description & Equation \\
\hline$c_{\text {mon }}$ & mol.cm ${ }^{-3}$ & Diffusing monosaccharides approximation & 22 \\
\hline$c_{l a c}$ & mol.cm ${ }^{-3}$ & Diffusing lactate approximation & 22 \\
\hline$c_{H_{2}}$ & mol.cm ${ }^{-3}$ & Diffusing hydrogen approximation & 22 \\
\hline$c_{a c}$ & mol.cm ${ }^{-3}$ & Diffusing acetate approximation & 22 \\
\hline$c_{\text {pro }}$ & mol.cm ${ }^{-3}$ & Diffusing propionate approximation & 22 \\
\hline$c_{b u}$ & mol.cm ${ }^{-3}$ & Diffusing butyrate approximation & 22 \\
\hline$c_{\mathrm{CH}_{4}}$ & mol.cm ${ }^{-3}$ & Diffusing methane approximation & 22 \\
\hline$c_{\mathrm{CO}_{2}}$ & mol.cm ${ }^{-3}$ & Diffusing carbon dioxyde approximation & 22 \\
\hline \multicolumn{4}{|c|}{ Model unknowns: speed approximations } \\
\hline Unknown & Unit & Description & Equation \\
\hline$u_{r}$ & $\mathrm{~cm}_{\mathrm{day}} \mathrm{d}^{-1}$ & Average mixture speed approximation & $25)-(26)$ \\
\hline$\tilde{u}_{s}$ & $\mathrm{~cm}^{\mathrm{day}}{ }^{-1}$ & Average speed for diffusing solutes approximation & 23 \\
\hline$\vartheta_{\mathrm{B}_{m o n}, r}$ & $\mathrm{~cm}^{\mathrm{day}}{ }^{-1}$ & Chemotactic speed for the $f_{\mathrm{B}_{m o n}}$ phase approx. & 23 \\
\hline$\vartheta_{\mathrm{B}_{l a}, r}$ & $\mathrm{~cm}^{\mathrm{day}}{ }^{-1}$ & Chemotactic speed for the $f_{\mathrm{B}_{l a}}$ phase approx. & 23 \\
\hline$\vartheta_{\mathrm{B}_{H_{2} a}, r}$ & cm.day $^{-1}$ & Chemotactic speed for the $f_{\mathrm{B}_{H_{2}} a}$ phase approx. & 23 \\
\hline$\vartheta_{\mathrm{B}_{H 2 m}, r}$ & $\mathrm{~cm}^{\mathrm{day}}{ }^{-1}$ & Chemotactic speed for the $f_{\mathrm{B}_{H_{2} m}}$ phase approx. & 23. \\
\hline \multicolumn{4}{|c|}{ Chemotactic potential approximations } \\
\hline Unknown & Unit & Description & Equation \\
\hline$\Upsilon_{m}$ & $\mathrm{~cm}^{2}$ & Chemotactic potential towards mucus approx. & 24 \\
\hline$\Upsilon_{p o l}$ & $\mathrm{~cm}^{2}$ & Chemotactic potential towards polysaccharides approx. & 24 \\
\hline$\Upsilon_{\text {mon }}$ & mol.cm ${ }^{-1}$ & Chemotactic potential towards monosaccharides approx. & 24 \\
\hline$\Upsilon_{l a}$ & mol.cm ${ }^{-1}$ & Chemotactic potential towards lactate approx. & 24 \\
\hline$\Upsilon_{H_{2}}$ & mol.cm ${ }^{-1}$ & Chemotactic potential towards $H_{2}$ approx. & 24 \\
\hline
\end{tabular}

Table Appendix A.3: Asymptotic model unknowns. The unknowns of the asymptotic model are listed with their units. The asymptotic model is numerically solved, giving an accurate approximation of the original model, with a light computational load.

- Figure Appendix B.2 presents the concentrations of microbial populations $\left(\mathrm{B}_{m o n}, \mathrm{~B}_{l a}, \mathrm{~B}_{H_{2} a}\right.$ and $\left.\mathrm{B}_{H_{2} m}\right)$ and SCFA (acetate, propionate and butyrate) in 6 different compartments (proximal lumen, proximal mucus, transverse lumen, transverse mucus, distal lumen, distal mucus) - see Section 3.2 .1 .

- Figure Appendix B.3 shows the spatial distribution of the bacterial activity defined by $\int_{0}^{R} s \sum_{i \in I_{\mathrm{B}}} F_{i}(s, z) d s$ for the six different tests compared with the reference state : low-fiber diet, high-fiber diet (see Section 3.2.1), without mucus degradation, without viscosity gradients (see Section 3.2 .2 ), with epithelial motility and with chemotaxis (see Section 3.2.3,

- Figure Appendix B.4 presents the longitudinal and the radial speeds with respect to $r$ at $z=10,61$, and $100 \mathrm{~cm}$, in the reference state and in the test cases without mucus degradation and without viscosity gradients (see Section 3.2.2, 


\begin{tabular}{|c|c|c|c|c|}
\hline \multicolumn{5}{|c|}{ Geometrical parameters } \\
\hline Parameter & Value & Unit & Description & Ref. \\
\hline$L$ & 154 & $\mathrm{~cm}$ & Total gut length & 29 \\
\hline$R$ & 2.5 & $\mathrm{~cm}$ & Gut radius & 29 \\
\hline$L_{\text {prox }}$ & 21 & $\mathrm{~cm}$ & Proximal colon length & 29 \\
\hline$L_{\text {trans }}$ & 70 & $\mathrm{~cm}$ & Transverse colon length & 29 \\
\hline$L_{\text {dist }}$ & 63 & $\mathrm{~cm}$ & Distal colon length & 29 \\
\hline$L_{\text {prox }, \text { water }}$ & 31.5 & $\mathrm{~cm}$ & Transverse colon length & {$[-]$} \\
\hline$L_{d i s t, w a t e r}$ & 51.2 & $\mathrm{~cm}$ & Distal colon length & {$[-]$} \\
\hline \multicolumn{5}{|c|}{ Diffusion parameters } \\
\hline Parameter & Value & Unit & Description & Ref. \\
\hline$\sigma$ & 0.8 & $\mathrm{~cm}^{2} \cdot \mathrm{d}^{-1}$ & Diffusion coefficient: mixture component & - \\
\hline$\sigma_{m o n}$ & 1.4 & $\mathrm{~cm}^{2} \cdot \mathrm{d}^{-1}$ & Diffusion coefficient: monosaccharides & - \\
\hline$\sigma_{l a}$ & 1.4 & $\mathrm{~cm}^{2} \cdot \mathrm{d}^{-1}$ & Diffusion coefficient: lactate & - \\
\hline$\sigma_{a c}$ & 1.4 & $\mathrm{~cm}^{2} \cdot \mathrm{d}^{-1}$ & Diffusion coefficient: acetate & - \\
\hline$\sigma_{\text {pro }}$ & 1.4 & $\mathrm{~cm}^{2} \cdot \mathrm{d}^{-1}$ & Diffusion coefficient: proprionate & - \\
\hline$\sigma_{b u t}$ & 1.4 & $\mathrm{~cm}^{2} \cdot \mathrm{d}^{-1}$ & Diffusion coefficient: butyrate & - \\
\hline$\sigma_{H_{2}}$ & 0.8 & $\mathrm{~cm}^{2} \cdot \mathrm{d}^{-1}$ & Diffusion coefficient: $H_{2}$ & - \\
\hline$\sigma_{\mathrm{CH}_{4}}$ & 2 & $\mathrm{~cm}^{2} \cdot \mathrm{d}^{-1}$ & Diffusion coefficient: $\mathrm{CH}_{4}$ & - \\
\hline$\sigma_{\mathrm{CO}_{2}}$ & 2 & $\mathrm{~cm}^{2} \cdot \mathrm{d}^{-1}$ & Diffusion coefficient: $\mathrm{CO}_{2}$ & - \\
\hline \multicolumn{5}{|c|}{ Viscosity parameters } \\
\hline Parameter & Value & Unit & Description & Ref. \\
\hline$\mu_{l, \min }$ & $0.864 \cdot 10^{3}$ & g.cm ${ }^{-1} \cdot d^{-1}$ & Water viscosity & 6 \\
\hline$\mu_{l, \max }$ & $36.7 \cdot 10^{3}$ & g.cm ${ }^{-1} \cdot \mathrm{d}^{-1}$ & Viscosity of dried mixture. & - \\
\hline$f_{l, t h r}$ & 0.5 & {$[-]$} & Viscosity threshold: liquid sigmoid & - \\
\hline$\alpha_{l}$ & 4 & {$[-]$} & Stiffness: liquid sigmoid & - \\
\hline$\mu_{m, \min }$ & $0.864 \cdot 10^{3}$ & g.cm ${ }^{-1} \cdot \mathrm{d}^{-1}$ & Minimal mucus viscosity & 6 \\
\hline$\mu_{m, \max }$ & $73.4 \cdot 10^{3}$ & g.cm ${ }^{-1} \cdot \mathrm{d}^{-1}$ & Maximal mucus viscosity & 6 \\
\hline$f_{m, t h r}$ & 0.0425 & {$[-]$} & Viscosity threshold: mucus sigmoid & - \\
\hline$\alpha_{m}$ & 14 & {$[-]$} & Stiffness: mucus sigmoid & - \\
\hline \multicolumn{5}{|c|}{ Chemotactic parameters } \\
\hline Parameter & Value & Unit & Description & Ref. \\
\hline$\lambda_{\mathrm{B}_{m o n}, m}$ & 82.64 & $d^{-1}$ & Mucus chemotactic coefficient for $\mathrm{B}_{m o n}$ & - \\
\hline$\lambda_{\mathrm{B}_{m o n}, p o l}$ & 28.32 & $d^{-1}$ & Polysaccharides coefficient for $\mathrm{B}_{m o n}$ & - \\
\hline$\lambda_{\mathrm{B}_{\text {mon }}, \text { mon }}$ & $9.32 \cdot 10^{3}$ & $\mathrm{~cm}^{3} \cdot \mathrm{mol}^{-1} \cdot \mathrm{d}^{-1}$ & Monosaccharides coefficient for $\mathrm{B}_{\text {mon }}$ & - \\
\hline$\lambda_{\mathrm{B}_{l a}, l a}$ & $0.85 \cdot 10^{7}$ & $\mathrm{~cm}^{3} \cdot \mathrm{mol}^{-1} \cdot \mathrm{d}^{-1}$ & Lactate chemotactic coefficient for $\mathrm{B}_{l a}$ & - \\
\hline$\lambda_{\mathrm{B}_{H_{2} a}, H_{2}}$ & $0.68 \cdot 10^{7}$ & $\mathrm{~cm}^{3} \cdot \mathrm{mol}^{-1} \cdot \mathrm{d}^{-1}$ & $H_{2}$ chemotactic coefficient for $\mathrm{B}_{H_{2} a}$ & - \\
\hline$\lambda_{\mathrm{B}_{H_{2} m}, H_{2}}$ & $0.68 \cdot 10^{7}$ & $\mathrm{~cm}^{3} \cdot \mathrm{mol}^{-1} \cdot \mathrm{d}^{-1}$ & $H_{2}$ chemotactic coefficient for $\mathrm{B}_{H_{2} m}$ & - \\
\hline \multicolumn{5}{|c|}{ Initial condition } \\
\hline Parameter & Value & Unit & Description & Ref. \\
\hline$f_{m \text { min }}^{\text {init }}$ & 0 & {$[-]$} & No mucus in lumen & 29 \\
\hline$f_{\text {min } \max }^{i n}$ & 0.05 & {$[-]$} & Maximal quantity of mucins & 29 \\
\hline$r_{m}$ & 2.25 & $\mathrm{~cm}$ & Threshold: initial condition & - \\
\hline$\alpha_{m}^{i n i t}$ & 8 & {$[-]$} & Stiffness: initial condition & - \\
\hline \multicolumn{5}{|c|}{ Other parameters and units } \\
\hline Parameter & Value & Unit & Description & Ref. \\
\hline$\rho$ & 1 & g.cm ${ }^{-1}$ & Mass density & - \\
\hline
\end{tabular}

Table Appendix A.4: Geometrical, diffusion, viscosity and initial condition parameters. The diffusion coefficients are calculated from order of magnitude of reported diffusion coefficients of the different compounds in water 38 multiplied by the corresponding diffusive ratio between water and mucus as reported in [4]. The viscosity of the dried mixture was set to half the mucus viscosity.

- Figure Appendix B.5 shows the longitudinal and the radial speeds with respect to $r$ at $z=10,61$, and $100 \mathrm{~cm}$, in the reference state and in the test cases with epithelial motility and with chemotaxis (see Section 3.2.3), 


\begin{tabular}{|c|c|c|c|c|}
\hline \multicolumn{5}{|c|}{ Source Function } \\
\hline Parameter & Value & Unit & Description & Ref. \\
\hline$\alpha$ & 0.113 & $\mathrm{~cm}^{3} \cdot \mathrm{mol}^{-1}$ & Multiplicative constant for unit conversion & \\
\hline$k_{P 1}$ & $1.20 \mathrm{e} 3$ & $d^{-1}$ & Maximum specific reaction rate for $\mathrm{P} 1$ & 29 \\
\hline$k_{P 2}$ & $1.20 \mathrm{e} 3$ & $\mathrm{~d}^{-1}$ & Maximum specific reaction rate for $\mathrm{P} 2$ & 29 \\
\hline$k_{P 3}$ & 7.92 & $d^{-1}$ & Maximum specific reaction rate for $\mathrm{P} 3$ & 29 \\
\hline$k_{P 4}$ & 103 & $d^{-1}$ & Maximum specific reaction rate for $\mathrm{P} 4$ & 29 \\
\hline$k_{P 5}$ & 108.837 & $\mathrm{~d}^{-1}$ & Maximum specific reaction rate for $\mathrm{P} 5$ & 29 \\
\hline$k_{P 6}$ & 22.581 & $d^{-1}$ & Maximum specific reaction rate for $\mathrm{P} 6$ & 29 \\
\hline$k_{P 7}$ & 0.01 & $\mathrm{~d}^{-1}$ & Maximum specific reaction rate for $\mathrm{P} 7$ & 29 \\
\hline$k_{P 8}$ & 0.01 & $\mathrm{~d}^{-1}$ & Maximum specific reaction rate for $\mathrm{P} 8$ & 29 \\
\hline$k_{P 9}$ & 0.01 & $d^{-1}$ & Maximum specific reaction rate for $\mathrm{P} 9$ & 29 \\
\hline$k_{P 10}$ & 0.01 & $d^{-1}$ & Maximum specific reaction rate for $\mathrm{P} 10$ & 29 \\
\hline$k_{P 11}$ & 200 & $d^{-1}$ & Maximum specific reaction rate for $\mathrm{P} 11$ & 29 \\
\hline$k_{P 12}$ & 200 & $d^{-1}$ & Maximum specific reaction rate for $\mathrm{P} 12$ & 29 \\
\hline$k_{P 13}$ & 200 & $\mathrm{~d}^{-1}$ & Maximum specific reaction rate for $\mathrm{P} 13$ & 29 \\
\hline$K_{h, P 11}$ & 0.0011 & mol.bar $^{-1}$ & Henry's Law coefficient & 29 \\
\hline$K_{h, P 12}$ & 0.0255 & mol.bar ${ }^{-1}$ & Henry's Law coefficient & 29 \\
\hline$K_{h, P 13}$ & $7.29 \mathrm{e}-4$ & mol.bar ${ }^{-1}$ & Henry's Law coefficient & 29 \\
\hline$R$ & 0.08314 & bar.mol ${ }^{-1} \cdot \mathrm{K}^{-1}$ & Ideal gas constant & 29 \\
\hline$T$ & 310.15 & K & Gut absolute temperature & 29 \\
\hline$\left[\mathrm{CH}_{4, g}\right]_{\infty}$ & $1.9106 e-10$ & mol.cm ${ }^{-3}$ & Gaseous $\mathrm{CH}_{4}$ steady state level & 29 \\
\hline$\left[\mathrm{CO}_{2, g}\right]_{\infty}$ & $1.19 e-5$ & mol.cm ${ }^{-3}$ & Gaseous $\mathrm{CO}_{2}$ steady state level & 29 \\
\hline$\left[H_{2, g}\right]_{\infty}$ & $3.6505 e-7$ & mol.cm ${ }^{-3}$ & Gaseous $\mathrm{H}_{2}$ steady state level & 29 \\
\hline$K_{x, P 1}$ & 20.265 & {$[-]$} & Half saturation constant (Comtois law) [-] & 29 \\
\hline$K_{x, P 2}$ & 0.265 & {$[-]$} & Half saturation constant (Comtois law) [-] & 29 \\
\hline$K_{s, P 3}$ & $2.6 e-6$ & mol.cm ${ }^{-3}$ & Half saturation for Monod law & 29 \\
\hline$K_{s, P 4}$ & $6.626 e-6$ & mol.cm ${ }^{-3}$ & Half saturation for Monod law & 29 \\
\hline$K_{s, P 5}$ & $1.7 e-6$ & mol.cm ${ }^{-3}$ & Half saturation for Monod law & 29 \\
\hline$K_{s, P 6}$ & $1.563 e-9$ & mol.cm ${ }^{-3}$ & Half saturation for Monod law & 29 \\
\hline$Y_{m, P 1}=Y_{l, P 1}$ & $8.850 e-3$ & {$[-]$} & Yield of component $\mathrm{m}$ in process 1 , & 29 \\
\hline$Y_{m o n, P 1}$ & $4.425 e-5$ & {$[-]$} & Yield of component mon in process 1 , & 29 \\
\hline$Y_{p o l, P 2}=Y_{l, P 2}$ & $8.850 e-3$ & {$[-]$} & Yield of component pol in process 2, & 29 \\
\hline$Y_{m o n, P 2}$ & $4.425 e-5$ & {$[-]$} & Yield of component mon in process 2 , & 29 \\
\hline$Y_{m o n, P 3}$ & $8.850 e-3$ & {$[-]$} & Yield of component mon in process 3 , & 29 \\
\hline$Y_{\mathrm{B} m o n}, P 3$ & 0.120 & {$[-]$} & biomass yield factor for $\mathrm{B}_{m o n}$ & 29 \\
\hline$Y_{l a, P 3}$ & $4.416 e-3$ & {$[-]$} & Yield of component la in process 3 , & 29 \\
\hline$Y_{a c, P 3}$ & $5.18 e-3$ & [-] & Yield of component ac in process 3 , & 29 \\
\hline$Y_{\text {pro }, P 3}$ & $2.124 e-3$ & {$[-]$} & Yield of component pro in process 3 , & 29 \\
\hline$Y_{\text {but }, P 3}$ & $2.389 e-3$ & {$[-]$} & Yield of component but in process 3 , & 29 \\
\hline$Y_{\mathrm{CO}_{2}}, \mathrm{P3}$ & $9.735 e-3$ & {$[-]$} & Yield of component $\mathrm{CO}_{2}$ in process 3 , & 29 \\
\hline$Y_{\mathrm{H}_{2}}, P 3$ & $1.274 e-2$ & {$[-]$} & Yield of component $\mathrm{H}_{2}$ in process 3 , & 29 \\
\hline$Y_{l a, P 4}$ & $8.850 e-3$ & {$[-]$} & Yield of component la in process 4 , & 29 \\
\hline$Y_{\mathrm{B}_{l a}, P 4}$ & 0.120 & {$[-]$} & biomass yield factor for $\mathrm{B}_{l a}$, & 29 \\
\hline$Y_{a c, P 4}$ & $1.177 e-3$ & {$[-]$} & Yield of component ac in process 4 , & 29 \\
\hline$Y_{\text {pro }, P 4}$ & $2.363 e-3$ & {$[-]$} & Yield of component pro in process 4 , & 29 \\
\hline$Y_{\text {but }, P 4}$ & $1.770 e-3$ & {$[-]$} & Yield of component but in process 4 , & 29 \\
\hline$Y_{\mathrm{CO}_{2}, P 4}$ & $4.717 e-3$ & {$[-]$} & Yield of component $\mathrm{CO}_{2}$ in process 4 , & 29 \\
\hline$Y_{H_{2}, P 4}$ & $3.540 e-3$ & {$[-]$} & Yield of component $\mathrm{H}_{2}$ in process 4 & 29 \\
\hline$Y_{H_{2}, P 5}$ & $8.850 e-3$ & {$[-]$} & Yield of component $H_{2}$ in process 5, & 29 \\
\hline$Y_{\mathrm{B}_{H_{2}} a}, P 5$ & 0.043 & {$[-]$} & biomass yield factor for $\mathrm{B}_{H_{2}, a}$, & 29 \\
\hline$Y_{a c, P 5}$ & $1.265 e-3$ & {$[-]$} & Yield of component ac in process 5 & 29 \\
\hline$Y_{\mathrm{CO}_{2}, P 5}$ & $-4.424 e-3$ & {$[-]$} & Yield of component $\mathrm{CO}_{2}$ in process 5 , & 29 \\
\hline$Y_{H_{2}, P 6}$ & $8.850 e-3$ & {$[-]$} & Yield of component $\mathrm{H}_{2}$ in process 6 , & 29 \\
\hline$Y_{\mathrm{B}_{H_{2}}}, P 6$ & 0.062 & {$[-]$} & biomass yield factor for $\mathrm{B}_{H_{2}, m}$, & 29 \\
\hline$Y_{C_{4}}, P 6$ & $8.407 e-4$ & {$[-]$} & Yield of component $\mathrm{CH}_{4}$ in process 6 , & 29 \\
\hline$Y_{\mathrm{CO}_{2}, P 6}$ & $-3.982 e-3$ & {$[-]$} & Yield of component $\mathrm{CO}_{2}$ in process 6 , & 29 \\
\hline$I_{p H, \min }$ & 5.5 & {$[-]$} & $p H$ in the proximal part of the gut & 29 \\
\hline$I_{p H, \max }$ & 6.8 & {$[-]$} & $p H$ in the distal part of the gut & 29 \\
\hline$I_{p H, l o w}$ & 5.8 & {$[-]$} & Threshold of inhibition & 29 \\
\hline$I_{p H, h i g h}$ & 6.7 & {$[-]$} & Threshold of inhibition & 29 \\
\hline
\end{tabular}

Table Appendix A.5: Parameter values of the source function.

- Figure Appendix B.6 displays a convergence graph of the Sobol indices for peristalsis, chemotaxis, viscosity and fiber intake with respect to the 


\begin{tabular}{|c|c|c|c|c|}
\hline \multicolumn{5}{|c|}{ Input fluxes on $\Gamma_{i n}$} \\
\hline Parameter & Value & Unit & Description & Ref \\
\hline$U_{i n}$ & $1.5 * 10^{3} /\left(2 \pi R^{2}\right)$ & $\mathrm{cm} \cdot \mathrm{d}^{-1}$ & Half average surface inflow & 29 \\
\hline$f_{m, i n}$ & $f_{m}(0, r, z)$ & {$[-]$} & Mucus density input & 29 \\
\hline$f_{\mathrm{B}_{m o n}, i n}$ & $6.1 e-5$ & {$[-]$} & Dietary $\mathrm{B}_{\text {mon }}$ input & 29 \\
\hline$f_{\mathrm{B}_{l a}, i n}$ & $2.03 e-5$ & {$[-]$} & Dietary $\mathrm{B}_{l a}$ density input & 29 \\
\hline$f_{\mathrm{B}_{H_{2} a}, i n}$ & $1.01 e-5$ & {$[-]$} & Dietary $\mathrm{B}_{H_{2} a}$ density input & 29 \\
\hline$f_{\mathrm{B}_{H_{2} m}, i n}$ & $1.01 e-5$ & {$[-]$} & Dietary $\mathrm{B}_{H_{2} m}$ density input & 29 \\
\hline$f_{\text {pol,in }}$ & $1.33 e-2$ (i.e. 25 g. $\mathrm{d}^{-1}$ ) & {$[-]$} & Dietary polysac. density input & 29 \\
\hline$c_{m o n, i n}$ & $3.33 e-5$ & mol.cm ${ }^{-3}$ & Dietary monosac. density input & 29 \\
\hline \multicolumn{5}{|c|}{ Boundary conditions on $\Gamma_{m}$} \\
\hline$f_{m}^{*}$ & 0.05 & {$[-]$} & Mucus threshold at the boundary & 29 \\
\hline$g_{m}$ & 3 & $\mathrm{~cm} \cdot \mathrm{d}^{-1}$ & Mucus production & 29 \\
\hline$g_{l, \max }$ & 17 & $\mathrm{~cm} \cdot \mathrm{d}^{-1}$ & Proximal liquid uptake & - \\
\hline$g_{l, \min }$ & 2 & $\mathrm{~cm} \cdot \mathrm{d}^{-1}$ & Distal liquid uptake & - \\
\hline$g_{l a, \max }$ & 16.85 & $\mathrm{~cm} \cdot \mathrm{d}^{-1}$ & Proximal mucosal lactate flow & 29 \\
\hline$g_{l a, \min }$ & 16.29 & $\mathrm{~cm} \cdot \mathrm{d}^{-1}$ & Transverse mucosal lactate flow & 29 \\
\hline$g_{a c, \max }$ & 25.28 & $\mathrm{~cm} \cdot \mathrm{d}^{-1}$ & Proximal mucosal acetate flow & 29 \\
\hline$g_{a c, \min }$ & 24.43 & $\mathrm{~cm} \cdot \mathrm{d}^{-1}$ & Transverse mucosal acetate flow & 29 \\
\hline$g_{b u, \max }$ & 17.23 & $\mathrm{~cm} \cdot \mathrm{d}^{-1}$ & Proximal mucosal butyrate flow & 29 \\
\hline$g_{b u, \min }$ & 16.81 & $\mathrm{~cm} \cdot \mathrm{d}^{-1}$ & Transverse mucosal butyrate flow & 29 \\
\hline$g_{\text {pro, } \max }$ & 20.49 & $\mathrm{~cm} \cdot \mathrm{d}^{-1}$ & Proximal mucosal propionate flow & 29 \\
\hline$g_{\text {pro,min }}$ & 19.93 & $\mathrm{~cm} \cdot \mathrm{d}^{-1}$ & Transverse mucosal propionate flow & 29 \\
\hline $\begin{array}{l}U_{p e r, r} \\
U_{\text {per }, z}\end{array}$ & $\begin{array}{l}-0.1 \\
-10\end{array}$ & $\begin{array}{l}\mathrm{cm} \cdot \mathrm{d}^{-1} \\
\mathrm{~cm} \cdot \mathrm{d}^{-1}\end{array}$ & $\begin{array}{c}\text { Radial peristaltic velocity } \\
\text { Longitudinal peristaltic velocity }\end{array}$ & $\begin{array}{l}{[-]} \\
{[-]}\end{array}$ \\
\hline
\end{tabular}

Table Appendix A.6: Parameters of the boundary fluxes.

number of runs used to build the data set (see Section 3.2.4). 


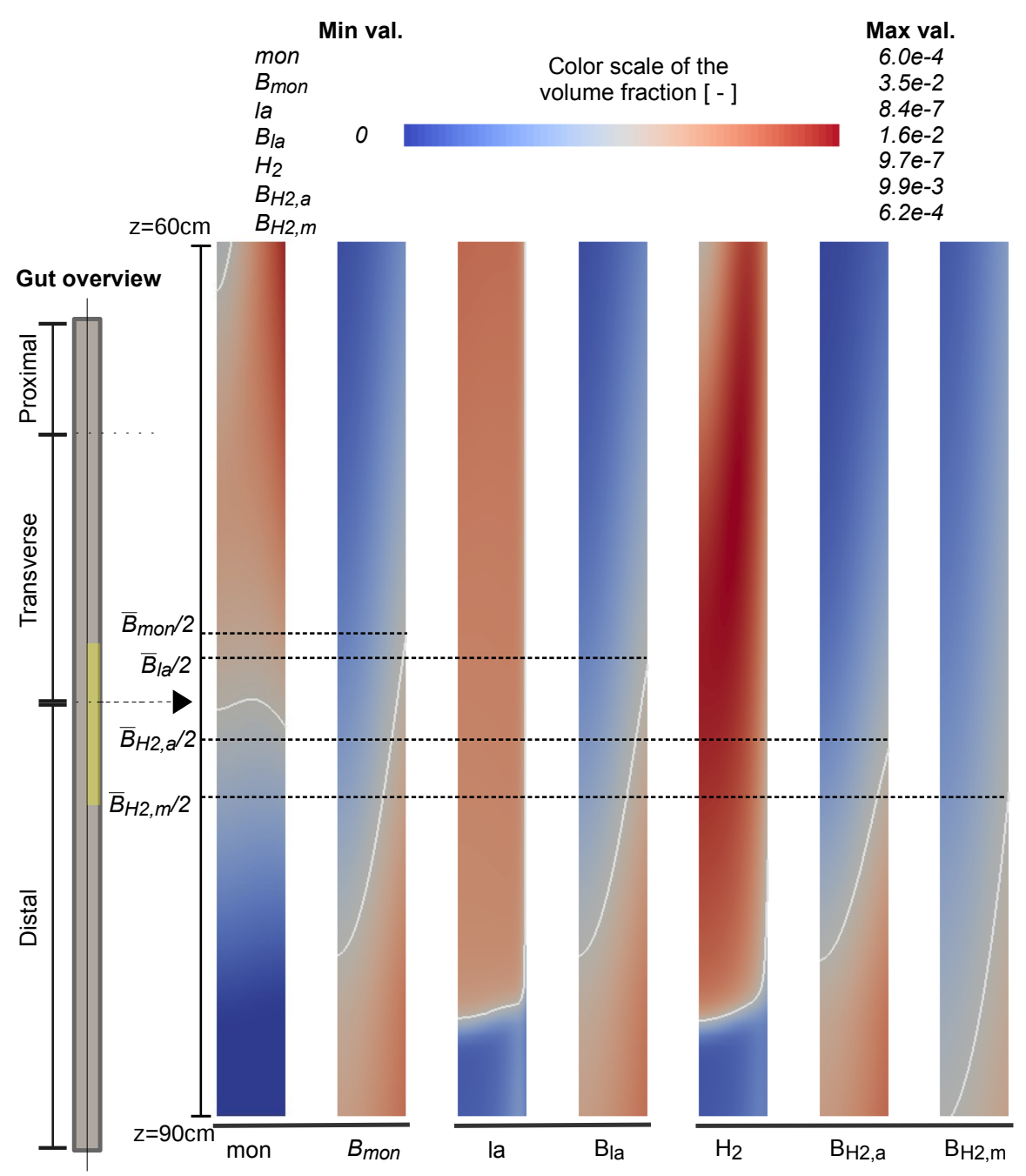

Figure Appendix B.1: Spatial distribution of the trophic chains in the transverse gut. The steady-state distribution of each bacterial population between $z=60$ and $z=90 \mathrm{~cm}$ is displayed together with its respective substrate. We represent also in each plot the isoline of half the maximal value. We indicate with dashed lines the intersection of this isovalue with the mucosa, in order to compare the longitudinal position of the bacterial fronts. We note that we represent half a plane of the cylindrical gut; the axis of the cylinder is the left boundary of the images, while the right boundary is the mucosa; the upper part of the images is the most proximal: the digestive flux is then directed from the top to the bottom of the figure. In the gut overview, the domain corresponding to the plots is indicated in yellow. 


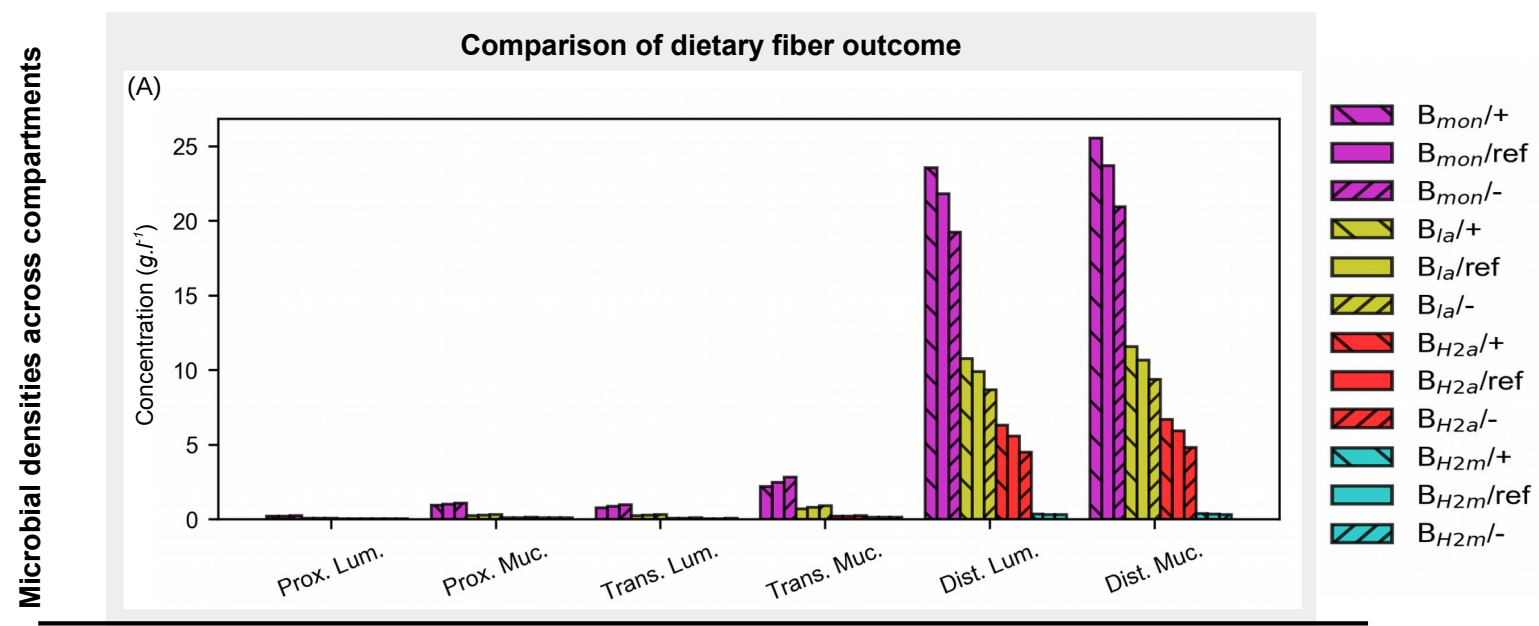

(B)

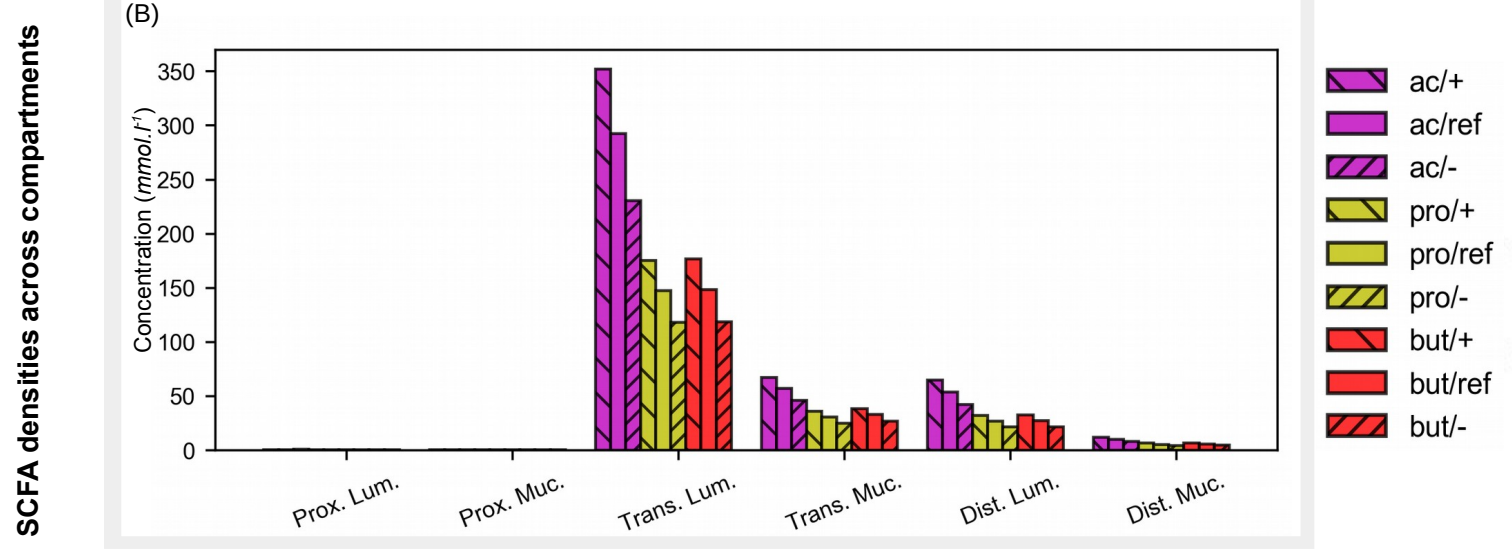

Figure Appendix B.2: Microbial and SCFA levels in the different compartments. We compare the microbial (A) and SCFA (B) levels during high-fiber(30\% increase of polysaccharide input, reverse slash hatches) or low-fiber (30\% decrease, slash hatches) diets with reference levels during normal diet (same polysaccharide input, plain boxes). The bacterial and SCFA levels are directly related to the fiber intake. 

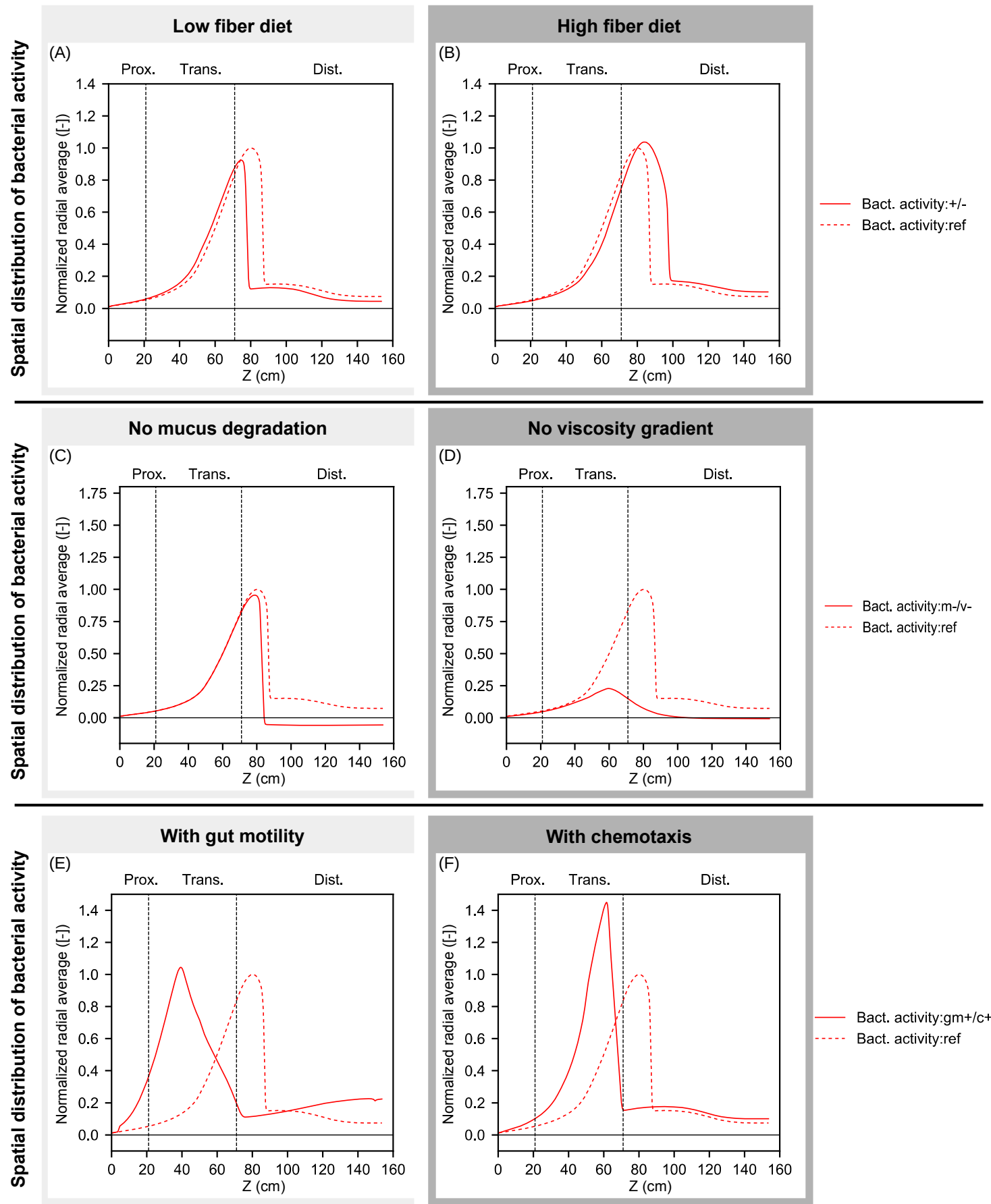

Figure Appendix B.3: Spatial distribution of the bacterial activity. We compare the microbial activity $z \mapsto \int_{0}^{R} s \sum_{i \in I_{\mathrm{B}}} F_{i}(s, z) d s$ with the reference state in each test case. 'ref': reference state. 'Bact activity:+/-': high/low fiber diet. 'Bact activity:m-/v-': without mucus metabolism/without viscosity gradient. 'Bact. activity: $\mathrm{gm}+/ \mathrm{c}+$ ': with gut motility/with chemotaxis. 


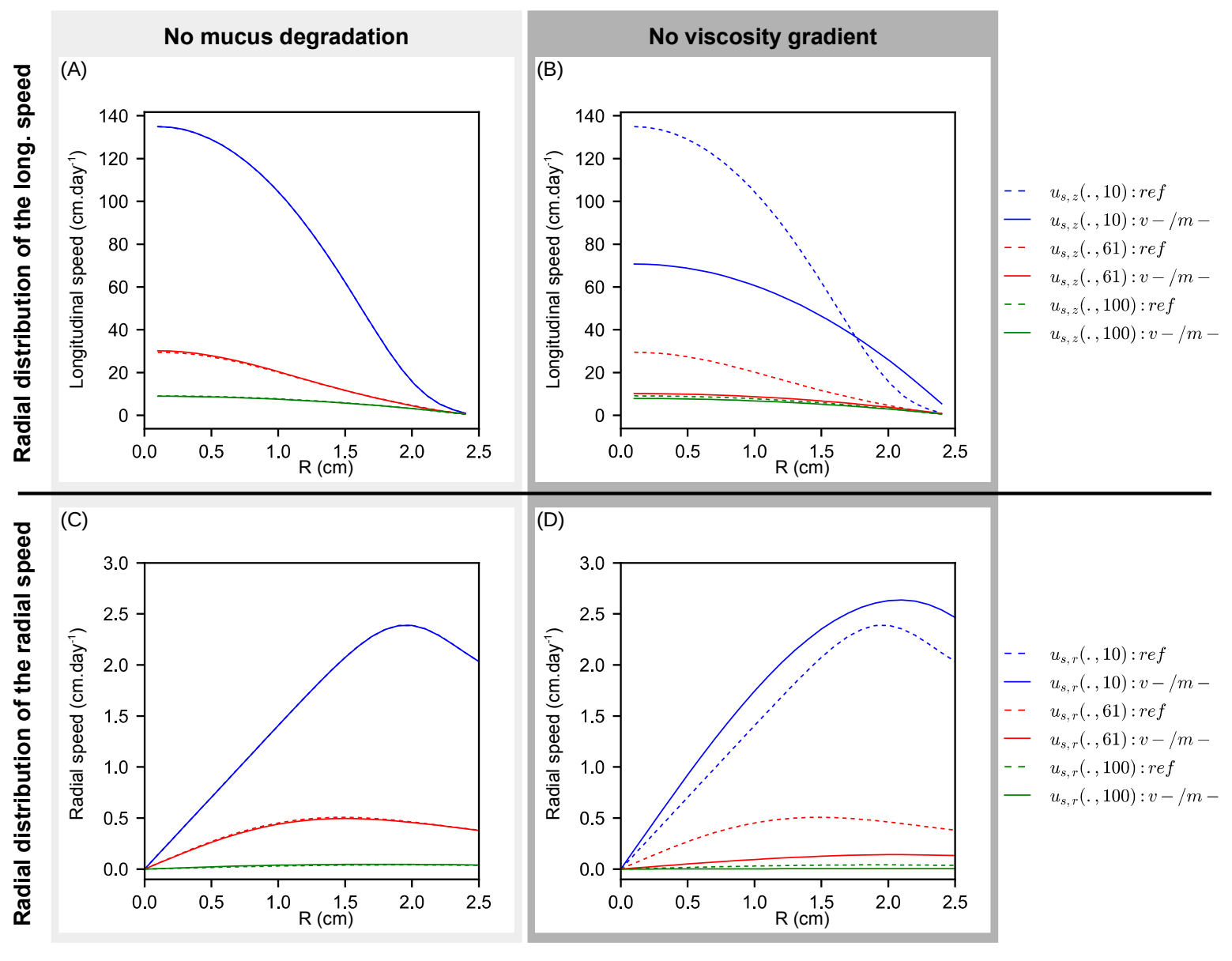

Figure Appendix B.4: Radial distribution of the longitudinal $\left(u_{s, z}\right)$ and radial $\left(u_{s, r}\right)$ speeds at $z=10,61$, and $100 \mathrm{~cm}$. To further explore the drivers of the bacterial activity, we display the radial distribution of the speed components with no mucus degradation $(m-, \mathrm{A}$ and $\mathrm{C}$ ) or a homogeneous viscosity $(v-, \mathrm{B}$ and D), compared with the reference of Fig. 3 (ref, dashed lines). 


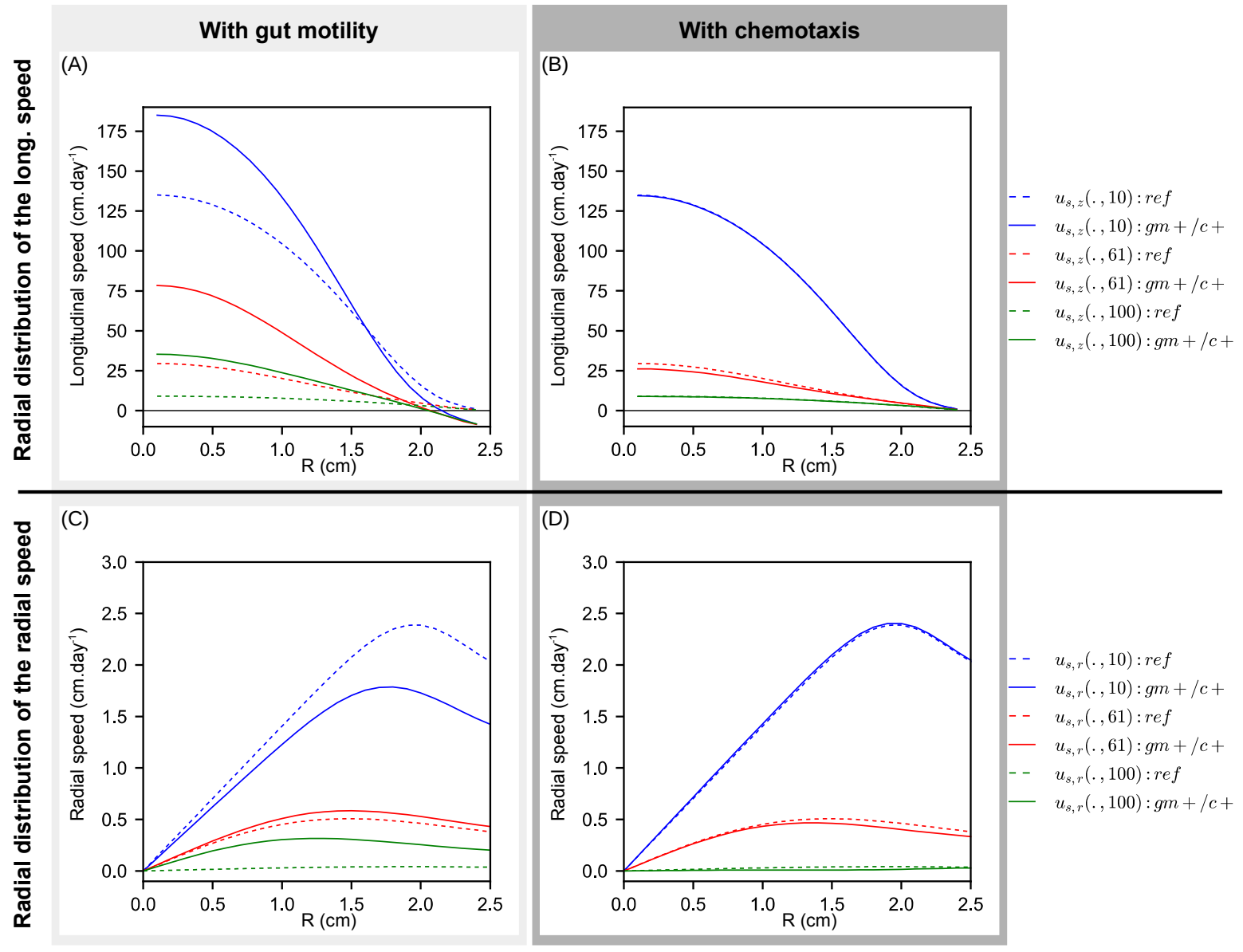

Figure Appendix B.5: Radial distribution of the longitudinal and radial speeds at $z=10$, 61 , and $100 \mathrm{~cm}$. To assess the effect of peristalsis and chemotaxis on the spatial structure of intestinal hydrodynamics, we display the radial distribution of the speed components at $z=10,61$, and $100 \mathrm{~cm}$ with gut motility $(g m+, \mathrm{A}$ and $\mathrm{C})$ or bacterial chemotaxis $(c+, \mathrm{B}$ and D), compared with the reference of Fig. 3 (ref, dashed lines). When the gut motility is active, negative speeds near the wall are observed, inducing a large increase of the longitudinal speed in the luminal part (blue line, A). Namely, if some material is transported upstream in the wall vicinity, as no outflux is possible in the proximal gut, a higher speed must be applied in the lumen to evacuate this additional material income in order to prevent volume expansion in the proximal gut. The difference of order of magnitude between the small upstream speed near the mucosa and the large downstream speed at $r=0$ is due to the cylindrical geometry. In cylindrical coordinates, the elementary volumes near the wall are larger than the luminal ones. Hence, for a same transport speed, the amount of fluid transported in the epithelial vicinity is higher than in the lumen. Consequently, a higher speed must be applied near the gut axis to insure volume conservation. 


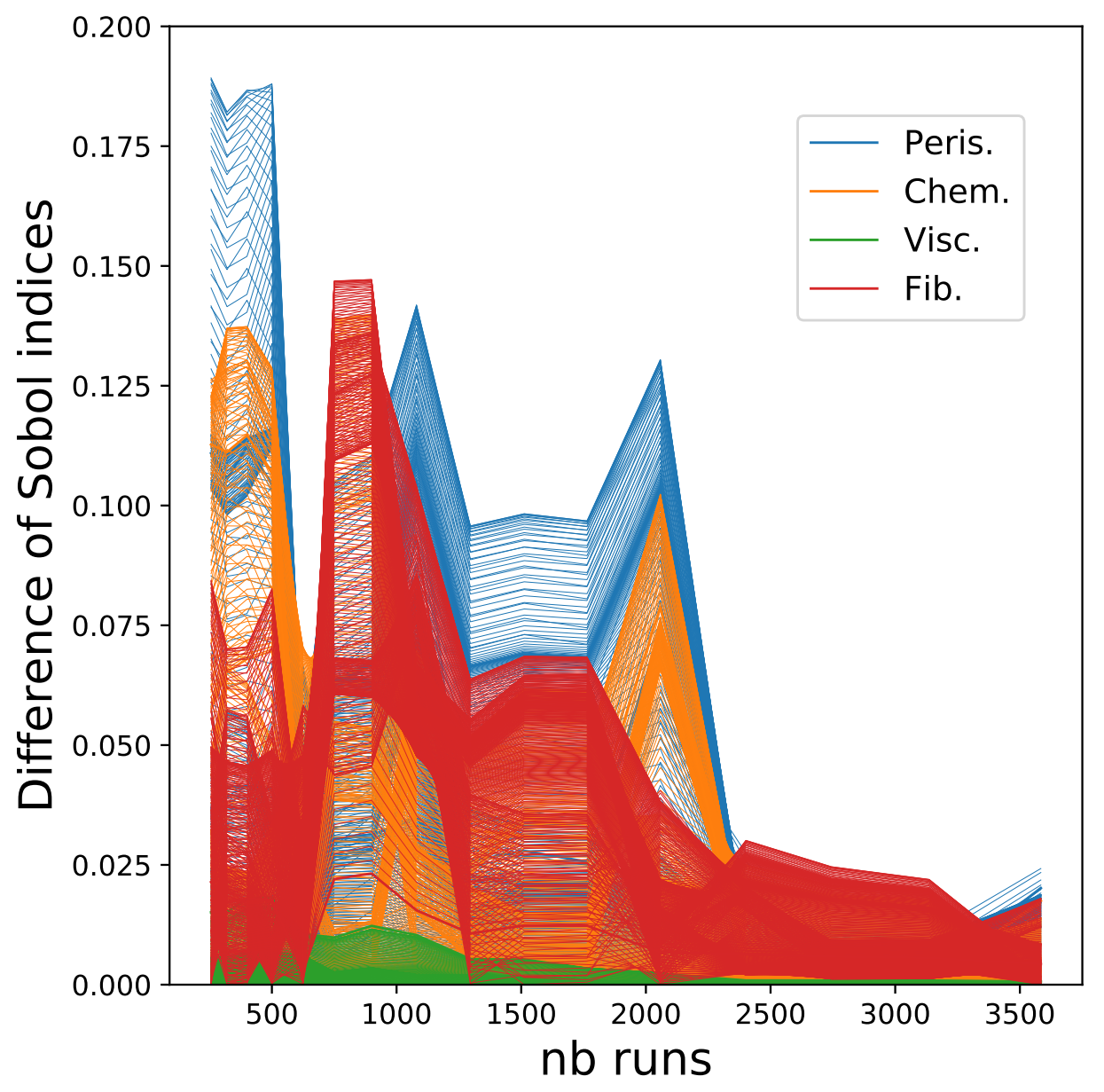

Figure Appendix B.6: Convergence graph of the Sobol indices computation. For each $z \in(0, L)$, and for each parameter, we plot the convergence curve of the corresponding Sobol index. To compute the convergence curves, we use complete factorial designs involving an increasing number of points (namely $256,320,400,500,625,750,900,1080,1296,1512,1764$ $2058,2401,2744,3136$ and 3584) together with the reference factorial plan that contains 4096 points. We plot the absolute value of the difference between the Sobol indices computed with the coarse designs and the reference design for varying number of runs involved in the coarse designs. 